\title{
Experimental Characterization of Turbulent Motions using Wall-Pressure Measurements in Low Reynolds Number Turbulent Boundary Layers
}

\author{
by \\ Jared Van Blitterswyk, B.Eng. \\ A thesis submitted to the \\ Faculty of Graduate and Postdoctoral Affairs \\ in partial fulfillment of the requirements for the degree of
}

Master's of Applied Science in Aerospace Engineering

Ottawa-Carleton Institute for Mechanical and Aerospace Engineering

Department of Mechanical and Aerospace Engineering

Carleton University

Ottawa, Ontario

September, 2016 


\section{(C)Copyright}

Jared Van Blitterswyk, 2016 
The undersigned hereby recommends to the

Faculty of Graduate and Postdoctoral Affairs

acceptance of the thesis

\section{Experimental Characterization of Turbulent Motions using Wall-Pressure Measurements in Low Reynolds Number Turbulent Boundary Layers}

submitted by Jared Van Blitterswyk, B.Eng.

in partial fulfillment of the requirements for the degree of

Master's of Applied Science in Aerospace Engineering

Professor Joana Rocha, Thesis Supervisor

Professor Ronald Miller, Chair,

Department of Mechanical and Aerospace Engineering

Ottawa-Carleton Institute for Mechanical and Aerospace Engineering

Department of Mechanical and Aerospace Engineering

Carleton University

September, 2016 


\section{Abstract}

The wall-pressure fluctuations induced by low Reynolds number turbulent boundary layers are experimentally studied using flush-mounted microphones. The spatial coherence of the energy is characterized using traditional time-averaged statistical descriptors. A novel analysis is developed, based on the wavelet transform, to study the organization of coherent turbulent events, and their corresponding wall-pressure signatures. This analysis identified that induced irrotational motions/entrained fluid, between neighbouring packets, have wall-pressure signatures below $100 \mathrm{~Hz}$, and packets of hairpin vortices contribute to the wall-pressure energy between $100 \mathrm{~Hz}$ and $250 \mathrm{~Hz}$. The packets contain a hierarchy of organized, well-defined events, which contribute to the wall-pressure fluctuations at frequencies above $250 \mathrm{~Hz}$. It is estimated that wallpressure signatures from packets can be retained for up to seven boundary layer thicknesses in the streamwise direction. The composition of events within hairpin packets depends on Reynolds number, showing a shift towards higher-frequency events, with increasing Reynolds number. 
To my parents, thank you for always supporting me. 


\section{Acknowledgments}

I would like to thank my supervisor, Dr. Joana Rocha, for her support, and the learning experiences over the past two years. I am grateful for the freedom to explore my research interests. Thank you for creating an open learning environment and allowing me to learn from my mistakes. This improved the quality of my work, and taught me several skills for future endeavours as a researcher.

I would also like to thank Dr. Metin Yaras for his encouragement and support during the final phases of this research, and always inspiring discussions about turbulence.

I would like to express my sincere gratitude to Alex Proctor, Ian Lloy and Kevin Sangster for their technical support and expertise. Without them, much of this research would not have been possible.

I thank my fellow students and friends, Matt and Ray for their support in the laboratory, and for the many stimulating discussions about my research along the way. These talks have, undoubtedly, improved many aspects of this work.

I am grateful to the Ontario Graduate Scholarship program and the Natural Sciences and Engineering Research Council of Canada for the generous financial support throughout my studies.

I am truly priviledged to have received such tremendous love and support from my family. I would not be where I am today without their encouragement. Thank you for your inspiration and always reminding me that dedication and a strong work ethic will 
pay off in the end.

Finally, but most importantly, I am very blessed to have had Jen Gatenby by my side during these past two years at Carleton. Without her, this thesis would not have materialized. Thank you for unwavering patience, understanding and support during this research. 


\section{Table of Contents}

Abstract iv

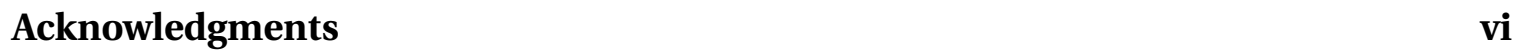

Table of Contents viii

List of Tables $\quad$ xi

List of Figures $\quad$ xiii

Nomenclature $\quad$ xxi

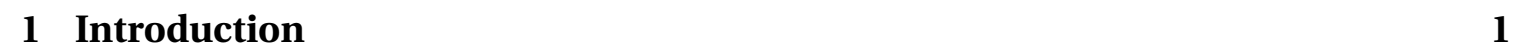

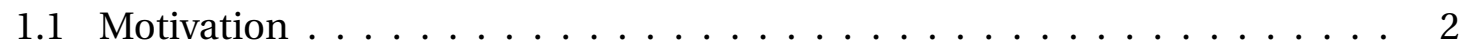

1.2 Literature Review $\ldots \ldots \ldots \ldots \ldots \ldots \ldots$

1.2.1 A review of coherent structures in wall-bounded flows. . . . . . . . 5

1.2.2 Single-point frequency spectrum of wall-pressure fluctuations $\ldots 9$

1.2.3 Spatial coherence of wall-pressure fluctuations . . . . . . . . . . 16

1.2.4 Identifying coherent structures in the turbulent boundary layer . . . 20

1.3 Objectives of the Thesis $\ldots \ldots \ldots \ldots \ldots$

1.4 Structure of the Thesis $\ldots \ldots \ldots \ldots \ldots \ldots \ldots \ldots$ 
\begin{tabular}{|ll|}
2 & Experimental Setup and Instrumentation \\
\hline
\end{tabular}

$2.1 \quad$ Experimental Facilities $\ldots \ldots \ldots \ldots \ldots \ldots \ldots$

2.2 Test Section Design and Modifications $\ldots \ldots \ldots \ldots$

$2.2 .1 \quad$ Design of the adjustable ceiling $\ldots \ldots \ldots$. . . . . . . . . . 35

2.2 .2 Test section floor $\ldots \ldots \ldots \ldots \ldots$

2.2 .3 Test panels $\ldots \ldots \ldots \ldots \ldots \ldots \ldots$. . . . . . . . . . . . . . . . . .

2.3 Experimental Techniques . . . . . . . . . . . . . . . . . . . 43

2.3.1 Hotwire anemometry system and traverse . . . . . . . . . . . . . 43

2.3 .2 Hotwire calibration $\ldots \ldots \ldots \ldots \ldots$. . . . . . . . . . . 45

2.3.3 Wall-pressure transducers, calibration and noise filtering . . . . . . . 46

2.4 Boundary Layer Trip Device Selection . . . . . . . . . . . . . . . . . . 50

\begin{tabular}{|ll|}
\hline 3 & Statistical Modeling of Wall-Pressure Fluctuations \\
\hline
\end{tabular}

3.1 Statistical Descriptors of Stationary Random Functions . . . . . . . . . . . . 53

3.2 Empirical Models for Studying Turbulence-Induced Wall-Pressure Fluctu-

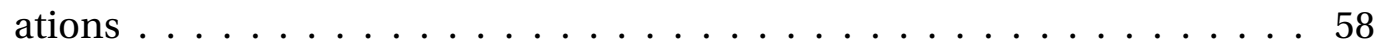

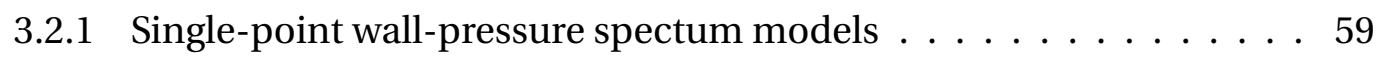

3.2 .2 Coherent energy of wall-pressure fluctuations . . . . . . . . . . . 63

3.3 Event Detection Using Wavelet Transform and Wavelet Cross-Correlation . 67

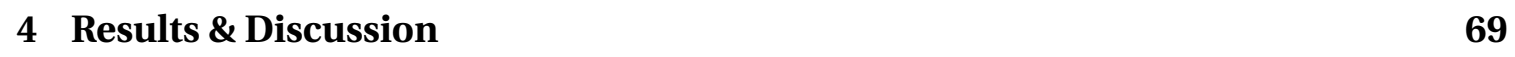

$4.1 \quad$ Single Point Wall-Pressure Spectrum: Original Configuration . . . . . . . . . 70

4.2 Mean Flow Pressure Gradient and Wall Boundary Layer . . . . . . . . . . . 73

4.3 Single-Point Wall-Pressure Spectrum - Final Configuration $\ldots \ldots$. . . . . 76

4.4 Wall-Pressure Spatial Coherence and Correlations . . . . . . . . . . . 81

4.4.1 Streamwise spatial correlations and convection velocity of wallpressure fluctuations . . . . . . . . . . . . . . 81 
4.4.2 Streamwise cross-spectrum and coherence lengths of wall-pressure

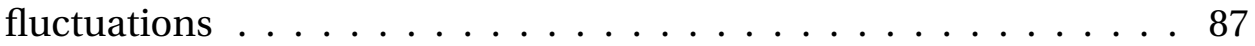

4.5 Wall-Pressure - Velocity Correlations $\ldots \ldots \ldots$. . . . . . . . . . 95

4.6 Coherent Event Detection Using a Wavelet Transform of Wall-Pressure Fluctuations . . . . . . . . . . . . . . . . . . . . 97

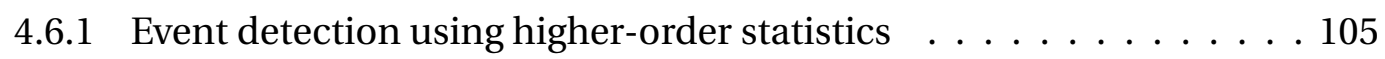

4.6.2 Comments on the use of higher-order statistics and wavelet transforms for event detection $\ldots \ldots \ldots$. . . . . . . . . . 121

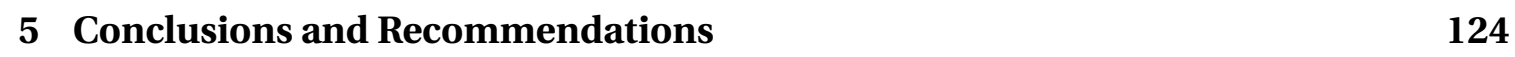

5.1 Summary of Measurements . . . . . . . . . . . . . . . . . . . . 124

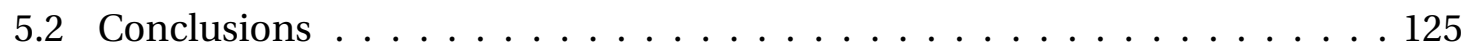

5.3 Research Contributions $\ldots \ldots \ldots$. . . . . . . . . . . . . . . . . . . . . . .

5.4 Recommendations for Future Work $\ldots \ldots \ldots$. . . . . . . . . . . 130

\begin{tabular}{ll}
\hline List of References & 134
\end{tabular}

\begin{tabular}{|lll}
\hline Appendix A Properties of Detected Event Packets & 147
\end{tabular} 


\section{List of Tables}

$1.1 \quad$ Regions and layers within a turbulent boundary layer and their defining properties (adopted from $[23])] \ldots \ldots \ldots \ldots \ldots$

1.2 Literature summary of measured features and normalization variables, for the distinct frequency regions of the single-point wall-pressure spectrum . 11

$4.1 \quad$ Measured boundary layer properties at $x=1.05 \mathrm{~m} \ldots \ldots$

4.2 Accuracy of empirical models for predicting experimental wall-pressure

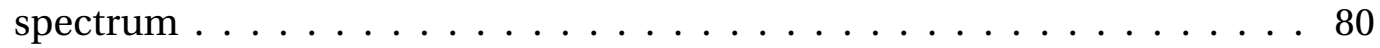

4.3 Streamwise spacing between each microphone in the array and the reference microphone $\ldots \ldots \ldots \ldots$. . . . . . . . . . . . . . 83

4.4 Coherence length parameters reported in the literature and derived from experimental measurements . . . . . . . . . . . . . . . . . . 94

4.5 Frequency bands used to investigate the composition of events contributing to periods of high activity identified by intermittency of wavelet coefficient energy. . . . . . . . . . . . . . . . . . . . . . . 107

4.6 Properties of event packets detected using intermittency of all wavelet scales between $55 \mathrm{~Hz}$ and $2,000 \mathrm{~Hz}$.

A.1 Summary of event packets detected using higher-order statistics for $R_{\theta} e_{\theta}=$

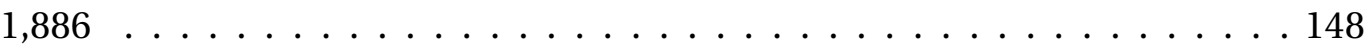


A.2 Summary of select event packets detected using higher-order statistics for

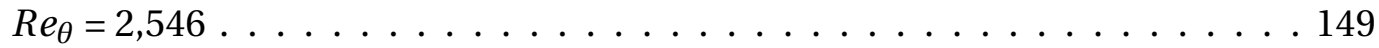




\section{List of Figures}

1.1 Typical mean velocity profile of a low Reynolds number turbulent bound-

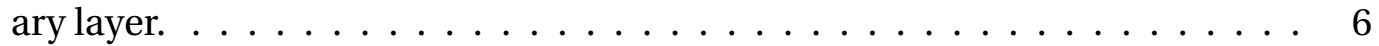

1.2 Schematic of idealistic, symmetric (left) and asymmetric (right) hairpin vortex structures. Arrows indicate the sense of circulation. . . . . . . . . 8

1.3 Typical wall-pressure spectrum of pressure fluctuations beneath a fully developed turbulent boundary layer (adapted from $[36 \mid$ ). . . . . . . . . . . . 12

1.4 Typical cross-correlation function for microphones, with various streamwise separations, from a common reference microphone. . . . . . . . . . . . 17

1.5 Cross-spectrum function between velocity in the buffer layer $\left(y^{+}=14(y / \delta\right.$ = 0.016)), and wall-pressure as measured by Snarksi \& Lupetow [74]. . . . . 23

1.6 The identification of four hairpins, aligned in a streamwise packet, as measured by Adrian et al. [19], using two-dimensional PIV. The velocity field is presented in a frame of reference moving at $0.8 U_{\infty}$. ' $y_{L}$ ' and ' $y_{B}$ ' denote the extents of the log-layer and buffer layer, respectively, and 'Q2' and 'Q4' denote classifications of events detected using the quadrant method. . . . . 25 
1.7 The instantaneous pressure field in a turbulent boundary layer from Ghaemi et al. [21]: a) full extent of the measurement domain, and b) a magnified view of the identified vortices near the wall. The labels 'A', 'B', and 'C' represent three vortex heads identified from the velocity field (vector lines shown in b), relative to a convecting frame of reference at

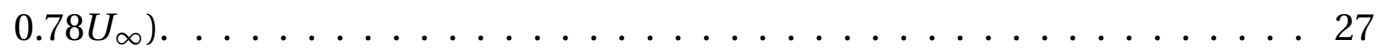

$2.1 \quad$ Diagram of wind tunnel test section in its initial configuration. . . . . . . . 33

2.2 Adjustable collar used to facilitate installation of the test section within the wind tunnel circuit. . . . . . . . . . . . . . . . . . . . 34

$2.3 \quad$ Schematic of assembled test section with new support cart (bold). . . . . . 35

2.4 Flexible ceiling designed for the test section: a) top view schematic of ceiling support layout and, b) completed ceiling structure prior to installation. 36

2.5 Schematic assembly of an adjustable ceiling support. . . . . . . . . . . 37

2.6 Schematic of the test section in its final configuration. . . . . . . . . . . . 39

2.7 Image of the interior of the test section. . . . . . . . . . . . . . . 39

2.8 Instrumented test panels for measurement of wall-pressure fluctuations:

a) 25-position, rectangular array and, b) 45-position, rotating array. . . . . . 40

2.9 Techniques for flush-mounting microphones: a) custom two-piece threaded capsules for panel 1 and, b) adhesive clay for panel 2 . . . . . . . . 41

2.10 Image of the rotating panel assembly installed in the test section floor. . . . 42

2.11 Schematic of the hotwire probe support structure . . . . . . . . . . . 43

2.12 Hotwire traverse system: a) custom frame to suspend three-axis traverse

(overall dimensions in inches) and, b) picture of assembled traverse system. 44

2.13 Typical hotwire calibration curve. The dashed line denotes a fourth-order calibration polynomial. . . . . . . . . . . . . . . . 45 
2.14 Distribution of cross-spectrum energy between freestream velocity fluctu-

ations and wall-pressure fluctuations;,$- U_{\infty}=7.6 \mathrm{~ms}^{-1} ;-, U_{\infty}=10.4$ $\mathrm{ms}^{-1} \ldots \ldots \ldots \ldots \ldots \ldots \ldots \ldots \ldots \ldots \ldots \ldots \ldots \ldots \ldots \ldots \ldots \ldots$

2.15 Measured boundary layer parameters compared to ideal, fully-developed

boundary layer trends: a) shape factor, b) wake strength, and c) skin friction coefficient. In a) and c) —, empirical trend from Coles [61]; in b) -, empirical trend from Finley [95]; $\mathbf{~ , ~ n a t u r a l ~ b o u n d a r y ~ l a y e r ; ~} \triangleright, 1$ trip wire; $\triangleleft, 4$ trip wires; $\bigcirc$, cylindrical pins; $\square, 3$ rectangular steps; $\triangle$, 3 Velcro strips;

ム, 6 Velcro strips. . . . . . . . . . . . . . . . . . . . . . . . . 52

$4.1 \quad$ Single-point wall-pressure spectrum at $x=1.10 \mathrm{~m}$ as measured using: a) a grid cap microphone, and b) a $0.5 \mathrm{~mm}$-diameter pinhole microphone; 一, $R e_{\theta} \approx 2,516 ;-, R e_{\theta} \approx 3,576 ;-, R e_{\theta} \approx 4,500 . R e_{\theta}$ estimated based on

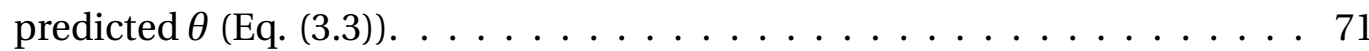

4.2 Mean pressure gradient profiles measured within the test section: a) streamwise, and b) spanwise at $x=1.05 \mathrm{~m} ; \circ, U_{\infty}=7.6 \mathrm{~m} / \mathrm{s} ; \triangle, U_{\infty}=10.4$

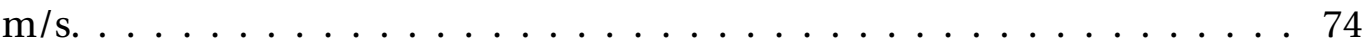

4.3 Boundary layer characterization over the center of the test panel $(x=$ $1.05 \mathrm{~m})$ : a) viscous-normalized, mean velocity profiles, and b) normalized Reynolds stress distributions; $\bigcirc, U_{\infty}=7.6 \mathrm{~m} / \mathrm{s} ; \triangle, U_{\infty}=10.4 \mathrm{~m} / \mathrm{s} . \mathrm{In}$ a): - - -, denotes $u^{+}=y^{+}$, and - , denotes the logarithmic law $\left(u^{+}=1 / 0.41 \ln \left(y^{+}\right)+\right.$

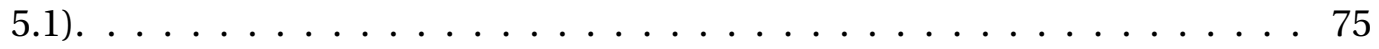

4.4 Single point wall-pressure spectrum as measured at $x=1.10 \mathrm{~m}$, and crossspectrum with reference microphone used for filtering: $-, R e_{\theta}=1,866$;

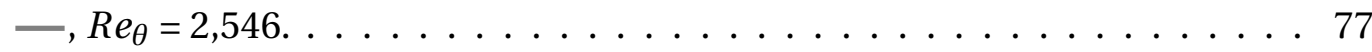


4.5 Experimental power spectra of wall-pressure: a) normalized by inner variables and compared to previous measurements by the authors and other literature, and b) compared to the models by Lowson [102], Robertson [54], Laganelli [55], Efimtsov [53], Smol'yakov [47], Goody [31], and Rackl \& Weston [30]; O, FC 3,386; $\diamond$, FC 4,487; $\square$, FC 6,025; $\boldsymbol{\nabla}$, VBR 2,517; $\mathbf{\Delta}$, SC 1,400; $\triangle$, GR 1,577; $\triangleright$, GR 2,953; $\triangleleft$, GR 3,509; $\nabla$, GR 6,$241 ;-, R e_{\theta}=1,886 ;-$, $R e_{\theta}=2,546 ; \cdots \cdot$, Efimtsov model; $-\cdot-$, Rackl \& Weston model; $\cdots \cdot$, Goody model; ---, Smol'yakov model, _-, Laganelli model, - - -, Lowson model, ..., Robertson model. The measurements from the literature are identified using a combination of letters, indicating the authors, and numbers, indicating the $R e_{\theta}$ of the data set: FC = Farabee \& Casarella [29], VBR = Van Blitterswyk \& Rocha [43], SC = Schewe [37], GR = Gravante et al. [33]. .. . 78

4.6 Schematic of microphone arrangements used to measure streamwise cross-correlations and cross-spectra. . . . . . . . . . . . . . . 82

4.7 Space-time correlations of wall-pressure fluctuations in the streamwise direction: a) -, $\xi / \delta \leq 1.0 ;-, 1.0<\xi / \delta \leq 2.0 ; \cdots, \xi / \delta>2.0$ and, b) $O, R e_{\theta}=1,886 ; \triangle, R e_{\theta}=2,546 ;$ dotted lines $=\exp \frac{-|\xi|}{L_{x}} ;$ dashed lines $=$ $A \cdot \exp \frac{-|\xi|}{L_{1}}+B \cdot \exp \frac{-|\xi|}{L_{2}} ;$ black and grey denote $R e_{\theta}=1,886$ and $R e_{\theta}=2,546$, respectively $\ldots \ldots \ldots \ldots \ldots \ldots \ldots \ldots \ldots$. . . . . . . . . . . . . . . . . .

4.8 Convection velocity derived from cross-correlations: $\bigcirc, \operatorname{Re}_{\theta}=1,886 ; \triangle$, $R e_{\theta}=2,546 ; \mathbf{\square}$, Willmarth \& Wooldridge [7]; $\diamond$, Leclerq \& Bohineust [40]; $\Delta$, Bull $[9] . . \ldots \ldots \ldots \ldots \ldots$. . . . . . . . . . . . . . 86

4.9 Streamwise cross-spectra between microphones of various streamwise separations as measured for $R e_{\theta}=2,593$ : a) as a function of frequency, and b) as a function of non-dimensional frequency, $\omega \delta / u_{\tau} ;-, \xi / \delta=0.73$; $\xi / \delta=1.45 ;-, \xi / \delta=2.54 ;--, \xi / \delta=3.63 . \ldots \ldots \ldots 8$ 
4.10 Streamwise cross-spectra between microphones of various streamwise separations: a) $R e_{\theta}=1,886$, b) $R e_{\theta}=2,546$, c) comparison of experimental cross-spectra with measurements from Leclerq \& Bohineust [40] at $\xi / \delta=$ $0.24, \xi / \delta=0.42$, and $\xi / \delta=0.90$, and d) comparison between experimental cross-spectra for $\xi / \delta=0.26$ and $\xi / \delta=0.53$ and predictions using the models proposed by Corcos [10] (Eq. (3.29)) (dashed lines) and, Leclerq \& Bohineust [40] (Eq. [3.33)) (solid lines). In a), and b): - $\xi / \delta=0.26$; $\xi / \delta=0.31 ;-, \xi / \delta=0.41 ; \cdots, \xi / \delta=0.53 ; \cdots, \xi / \delta=0.85 ; \cdots, \xi / \delta=1.14 ; \cdots, \cdots$, $\xi / \delta=1.40 ;-=-, \xi / \delta=2.02 ;--^{-}, \xi / \delta=3.20 ;-\cdot-, \xi / \delta=4.37$. In c) solid lines, $\xi / \delta=0.26$, dotted lines, $\xi / \delta=0.53$, and dashed lines, $\xi / \delta=0.85$; black and grey denote $R e_{\theta}=1,886$ and $R e_{\theta}=2,546$, respectively. . . . . . . . . . . 89

4.11 Coherence lengths analysis for $R e_{\theta}=1,886, R e_{\theta}=2,549$ and $R e_{\theta}=2,593$ from Van Blitterswyk \& Rocha [67]: a) an example of the normalized crossspectrum amplitude as measured by each microphone in the rotating array, used to derive the coherence length at $82 \mathrm{~Hz}$ for $R e_{\theta}=1,886$, and b) coherence lengths for all frequencies up to $1,500 \mathrm{~Hz} ;-, R e_{\theta}=1,886$; $R e_{\theta}-2,546 ; \cdots, R_{\theta}=2,593$. The dashed line in a) denotes the exponential fitting used to extract the coherence length. . . . . . . . . . . . . . . 93

4.12 Comparison of measured coherence length distributions to empirical models proposed by Corcos [10] (Eq. [3.31p) and Efimtsov [52] (Eq. (3.32)): 一, $R e_{\theta}=1,886 ;-, R e_{\theta}=2,546$. Dashed and dotted lines denote predictions with the Corcos model and Efimtsov model, respectively. . . . . . . . . 94 
4.13 Cross-spectrum between streamwise velocity fluctuations and pressure fluctuations measured at different regions in the boundary layer at $x=1.10$ m: a) $R e_{\theta}=1,886$, and b) $R e_{\theta}=2,546 .-, y / \delta=0.007 ;-, y / \delta=0.010 ;-$, $y / \delta=0.019 ;-\cdots, y / \delta=0.067 ;=-\cdot, y / \delta=0.17 ;--, y / \delta=0.24 ; \cdots \cdots, y / \delta=0.74 ;$ $\ldots \ldots, y / \delta=1.40 . \ldots \ldots \ldots \ldots \ldots \ldots \ldots$

4.14 Streamwise microphone arrays used to characterize: a) the convective nature of higher frequency events (Type 4, 5 and 6 events), and b) the convective nature of lower frequency events (Type 1, 2 and 3 events) and event packets. . . . . . . . . . . . . . . . . . 98

4.15 Wavelet cross-correlation analysis of wall-pressure fluctuations measured by two microphones with a streamwise separation of $0.25 \delta$, under conditions of $R e_{\theta}=2,546$ : a) real part of wavelet cross-correlation coefficients, $w_{12}(s, \tau)$ (Eq. (3.36)), and b) traditional time-averaged cross-correlation, $r_{p p}(\xi, 0, \tau)$, as defined by Eq. $(3.9) . \ldots \ldots \ldots \ldots \ldots 9 . \ldots \ldots$

4.16 Probability density function of time delay between successive events for various flow conditions and microphone placements: a, Camussi et al. [17]; $\triangle, R e_{\theta}=2,546-\xi=8.6 \mathrm{~mm} ; \nabla, R e_{\theta}=2,546-\xi=18 \mathrm{~mm}, \triangleleft, R e_{\theta}=$ $1,886-\xi=8.6 \mathrm{~mm} ; \triangleright, R_{\theta}=1,886-\xi=18.6 \mathrm{~mm}$. Dashed line denotes a pure exponential decay. . . . . . . . . . . . . . . . . . 101

4.17 Streamwise cross-correlation, $r_{p p}(\xi, 0, \tau)$, and normalized convection velocity, $U_{c}$, of events detected using the wavelet cross-correlation analysis for $R e_{\theta}=2,546$ : a) $r_{p p}(\xi, 0, \tau)$, and b) $U_{c}$ for events in the $125-250 \mathrm{~Hz}$ frequency band, c) $r_{p p}(\xi, 0, \tau)$, and d) $U_{c}$ for events in the $250-500 \mathrm{~Hz}$ frequency band. Dashed lines in place to emphasize trends. . . . . . . . . . . . 102 
4.18 Fourier-based cross-spectrum $(-, \xi / \delta=0.25, \cdots, \xi / \delta=0.53)$ compared with the conditional sampling counterparts (open symbols): a) $R e_{\theta}=$ 1,886, and b) $R e_{\theta}=2,546$. . . . . . . . . . . . . . . . . . . . . 104

4.19 Probability density function of time delay for successive events and various flow conditions using higher-order statistics for event detection: Camussi et al. [17]; $\bigcirc, R e_{\theta}=1,886 ; \triangle, R e_{\theta}=2,546$. Dashed line denotes pure exponential decay. . . . . . . . . . . . . . . . . . . . 107

4.20 Event detection using the continuous wavelet transform for $R e_{\theta}=2,546$ :

a) sample pressure signal, and b) - , the kurtosis signature based on all scales in the wavelet transformation. - - -, represents the Gaussian noise threshold for event localization. . . . . . . . . . . . . . . . . . . 108

4.21 Comarison of kurtosis signature computed using all wavelet scales (a), compared to the signatures computed over each frequency band (b), to illustrate the multi-scale nature of the large-scale events. In (b): —, Type $1 ;$-, Type $2 ; \ldots . .$, Type $3 ; \cdots . . .$, Type $4 ;$ - - -, Type 5. . . . . . . . . . . . . . . . . 109

4.22 The association of (a) frequency band kurtosis signatures, with (b) features

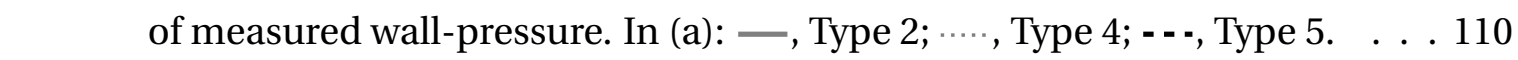

4.23 a) Schematic representation of an idealistic two-hairpin packet, and b) expected features of the wall-pressure signature. Dashed line in b) denotes an inclined shear layer.$\ldots \ldots \ldots \ldots \ldots \ldots$

4.24 Probability density function of event length as a function of frequency: $\bigcirc$, $R e_{\theta}=1,886 ; \triangle, R e_{\theta}=2,546$. Dotted and dashed lines denote power law fittings for $R e_{\theta}=1,886$ and $R e_{\theta}=2,546$, respectively. . . . . . . . . . . . . 114 
4.25 Average convection velocity, as a function of frequency, for events detected using higher-order statistics: a) $R e_{\theta}=1,886$, and b) $R e_{\theta}=2,546 ; \triangle$, Salze et al. [42]; $\nabla$, Farabee \& Casarella [29]; $\triangleright$, Arguillat et al. [3]; -, Smol'yakov model [105]; - - , Leclerq \& Bohineust model [40]. . . . . . . . . . . . . . . . 116

4.26 Probability density function of event packet composition: ${ }^{-}{ }^{-}, R e_{\theta}=1,886$; $-\triangle, R e_{\theta}=2,546 . \ldots \ldots \ldots \ldots \ldots \ldots$ 


\title{
Nomenclature
}

\author{
$A, B \quad$ Cross-correlation model constants \\ $A_{c}, B_{c} \quad$ Streamwise, spanwise coherence functions - Corcos cross-spectrum model \\ $a_{1}, a_{2}, a_{3} \quad$ Empirical constants - Efimtsov coherence length model \\ $a, b, c \quad$ Empirical constants - Smoly'akov convection velocity model \\ $C_{f} \quad$ Skin-friction coefficient \\ $C_{p} \quad$ Static pressure coefficient \\ $d \quad$ Effective microphone diameter [m] \\ $d^{+} \quad$ Normalized effective microphone diameter [m] \\ $E_{a} \quad$ Hotwire voltage uncorrected for temperature effects [V] \\ $E_{c} \quad$ Hotwire voltage corrected for temperature effects [V] \\ $F_{c} \quad$ Laganelli transformation function \\ $F_{x} \quad$ Probability density function of random variable ' $x$ ' \\ $f \quad$ Frequency $[\mathrm{Hz}]$ \\ $f_{b} \quad$ Wavelet bandwidth $\left[\mathrm{rads}^{-1}\right]$
}




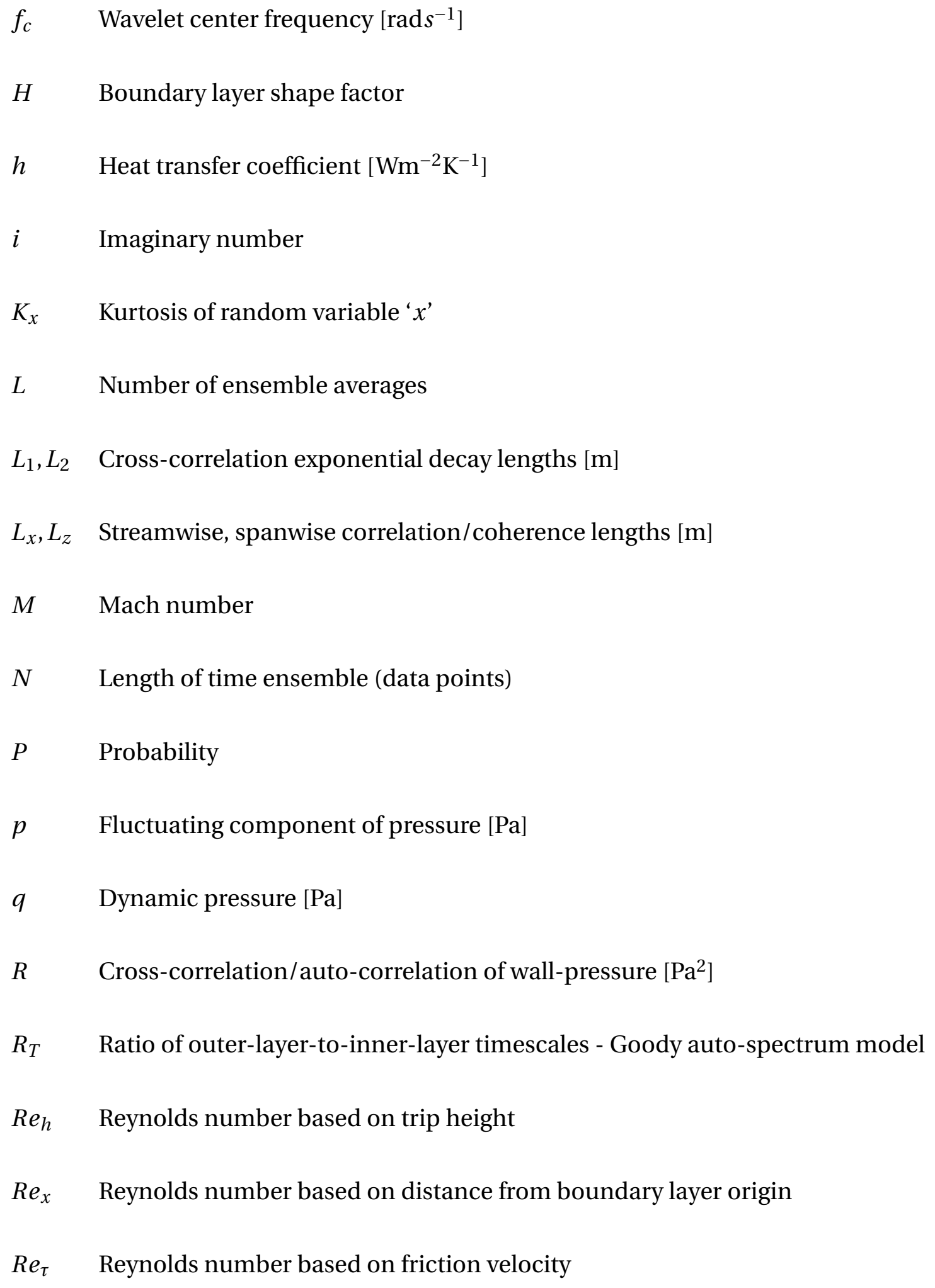


$R e_{\theta}$ Reynolds number based on momentum thickness

$r \quad$ Normalized cross-correlation/auto-correlation

Sh Strouhal number

$S \quad$ Pressure power spectral density $\left[\mathrm{Pa}^{2}\right]$

$S_{x} \quad$ Skewness of random variable ' $x$ '

$s \quad$ Wavelet scale

$T \quad$ Time period $[\mathrm{s}]$

$T_{a} \quad$ Ambient static temperature $[\mathrm{K}]$

$T_{o} \quad$ Calibration temperature $[\mathrm{K}]$

$T_{w} \quad$ Hotwire temperature $[\mathrm{K}]$

$t \quad$ Time [s]

$U \quad$ Energy in spectrum window function

$U_{c} \quad$ Convection velocity $\left[\mathrm{ms}^{-1}\right]$

$U_{\infty} \quad$ Mean freestream velocity $\left[\mathrm{ms}^{-1}\right]$

$u \quad$ Fluctuating component of streamwise velocity [ms $\left.{ }^{-1}\right]$

$u_{\tau} \quad$ Friction velocity $\left[\mathrm{ms}^{-1}\right]$

$u^{+} \quad$ Viscous normalized mean streamwise velocity

$v \quad$ Fluctuating component of wall-normal velocity $\left[\mathrm{ms}^{-1}\right]$ 
W Window function

$w \quad$ Wavelet transform of pressure

$x, y, z \quad$ Cartesian coordinates (streamwise, wall-normal, and spanwise directions, respectively)

$x^{+} \quad$ Viscous normalized event length

$y^{+} \quad$ Viscous normalized wall-normal distance

$\alpha \quad$ Empirical constant - Efimtsov auto-spectrum model

$\alpha_{L} \quad$ Empirical constant - Leclerq \& Bohineust cross-spectrum model

$\alpha_{x}, \alpha_{y} \quad$ Exponential decay constants - Corcos cross-spectrum model

$\beta \quad$ Empirical constant - Efimtsov auto-spectrum model

$\beta_{c} \quad$ Clauser pressure parameter

$\beta_{L} \quad$ Empirical constant - Leclerq \& Bohineust cross-spectrum model

$\Gamma \quad$ Cross-spectrum of wall-pressure $\left[\mathrm{Pa}^{2} \mathrm{~Hz}^{-1}\right]$

$\gamma \quad$ Cross-spectrum of wall-pressure, adiabatic constant of air

$\Delta \quad$ Relative change in quantity

$\delta \quad$ Boundary layer thickness [m]

$\delta^{*} \quad$ Boundary layer displacement thickness [m]

$\eta \quad$ Spanwise separation between two microphones [m]

xxiv 
$\theta \quad$ Boundary layer momentum thickness [m]

$\Lambda \quad$ Boundary layer equilibrium parameter

$\lambda \quad$ Wavelength [m]

$v \quad$ Kinematic viscosity $\left[\mathrm{m}^{2} \mathrm{~s}^{-1}\right]$

$\xi \quad$ Streamwise separation between two microphones [m]

$\Pi \quad$ Boundary layer wake strength

$\rho \quad$ Density of air $\left[\mathrm{kgm}^{-3}\right]$

$\sigma_{x} \quad$ Standard deviation of variable ' $\mathrm{x}$ ' [units of $\mathrm{x}$ ]

$\tau \quad$ Time delay $[\mathrm{s}]$

$\tau_{w} \quad$ Wall shear stress $[\mathrm{Pa}]$

$\Phi_{p} \quad$ Single-sided power spectral density of pressure $\left[\mathrm{Pa}^{2} \mathrm{~Hz}^{-1}\right]$

$\Phi_{u} \quad$ Single-sided power spectral density of velocity $\left[\mathrm{m}^{2} \mathrm{~s}^{-2} \mathrm{~Hz}^{-1}\right]$

$\Psi \quad$ Mother wavelet function

$\omega \quad$ Frequency $\left[\mathrm{rads}^{-1}\right]$ 


\section{List of Abbreviations}

'HAPP' High amplitude pressure peak

'PIV' Particle image velocimetry

'PDF' Probability density function

'PSD' Power spectral density

'RMS' Root mean square

‘TBL’ Turbulent boundary layer

'VFD' Variable frequency drive

'VITA' Variable-interval time-averaging 


\section{Chapter 1}

\section{Introduction}

A thorough understanding of wall-pressure fluctuations, produced beneath a turbulent boundary layer (TBL), is important for properly modeling flow-induced vibrations and sound radiation into the cabins of commercial aircraft, submarines, trains and road vehicles [1-4]. The extensive use of lightweight and flexible structures in the design of these vehicles has resulted in increased attention to the physical mechanisms, which generate the unsteady pressure fluctuations at the wall, causing these flexible structures to radiate sound inside the vehicle. These sound pressure levels can be intense enough to cause passenger discomfort, communication interference, or malfunction of sensitive equipment [1, 5]. The pressure field on the flow side of the structure consists of the unsteady pressure field beneath the TBL, and acoustic pressures originating from structural vibrations [6]. The computational expenses associated with resolving turbulence at Reynolds numbers of practical relevance makes fully-coupled simulations prohibitive and therefore, statistical models are used instead. Wall-pressure excitations are typically modeled as those which would be observed on a rigid wall by assuming a weak coupling between the turbulent flow field and the small amplitude motions of the structure. These models are then applied as forcing functions in simulations to predict the forced response of the structure. 
The advancement of structural designs for noise reduction requires a complete understanding of the relationships between turbulent motions in the boundary layer, and wall-pressure fluctuations. Early efforts focused on analyzing the average statistical properties of wall-pressure point measurements in the space, frequency and time domains [7-9]. While initially useful for understanding the mean convective and spatial coherence of the wall-pressure fluctuations, the inherent averaging associated with time-averaged analyses limited the ability to establish definitive relationships between coherent turbulent events and their corresponding wall-pressure signatures. Recent advancements in instrumentation, for wall-pressure and fluid flow-field measurements, have enabled more detailed accounts to be made of wall-pressure and velocity fluctuations in TBLs. New approaches for data analysis have been conceived to compliment these more detailed measurements, and study the relationships between wall-pressure and turbulence in greater detail. To that end, this thesis focuses on the use of both conventional and novel statistical analyses for modeling the wall-pressure field beneath low Reynolds number TBLs, using point measurements of wall-pressure.

\subsection{Motivation}

The development of deterministic models for the spatial characteristics of the turbulent wall-pressure excitation is not an intuitive problem due to the wide range of turbulent motions contributing to the pressure field. To be useful as a design tool, such models must be sufficiently simple, yet comprehensive enough to capture the physical mechanisms responsible for generating wall-pressure fluctuations. In the 1960's, one of the first empirical models for the spatial properties of wall-pressure fluctuations was proposed by Corcos [10], which was derived using measurements of the unsteady wall-pressure 
field at discrete points. His model (Eq. (1.1) ) separates the wall-pressure power spectrum, $S$, into functions describing the frequency distribution of wall-pressure energy at a single point, using the power spectral density function $\phi(\omega)$, and separate functions for the spatial coherent power of that energy in the streamwise, $A\left(\omega \xi / U_{c}\right)$, and spanwise, $B\left(\omega \eta / U_{c}\right)$, directions. In Eq. $1.1, \omega$ denotes frequency $(2 \pi f), U_{c}$ denotes the average convection velocity of the wall-pressure signature, and $\xi$ and $\eta$ represent the spatial separations between a reference microphone and another point in space, in the streamwise and spanwise directions, respectively.

$$
S(\omega, \xi, \eta)=\phi(\omega) A\left(\omega \xi / U_{c}\right) B\left(\omega \eta / U_{c}\right) e^{-i \omega \xi / U_{c}}
$$

Several models for the single-point spectrum have been proposed, each with significantly different forms and characteristic features leaving some uncertainty between models. The majority of models for the spatial coherence adopt the exponential form, proposed by Corcos [10], as the parent function; however, the coherence of lowfrequency pressure fluctuations $\left(\omega \delta / u_{\tau} \leq 50\right.$ where; $\delta$, and $u_{\tau}$, denote boundary layer thickness, and friction velocity, respectively), which do not decay exponentially, have yet to be accurately modeled. The uncertainty between studies is largely attributed to variations in instrumentation, facility characteristics, and Reynolds number [11]. Therefore, further investigation is required to assess the accuracy of available models.

Single-point frequency spectrum models, conventional space-time correlations, and Fourier-based cross-spectrum correlations, or 'coherence', have been used to infer relationships between wall-pressure and average characteristics of turbulent events. However, these techniques lose all locality in the pressure signal making it difficult to relate characteristics of these statistical descriptors to physical features in the flow [12]. This limitation is especially important in the context of studying the physical mechanisms of 
wall-pressure generation. Many empirical models are developed using measurements collected in wind tunnels, which are limited to low or moderate flow speeds. In these conditions the mean boundary layer properties can appear similar to high Reynolds number flows, but the underlying, time dependent characteristics of the wall-pressure fluctuations can be quite different. An alternative approach is to use the wavelet transform, which scales and shifts a baseline function, that is localized in space and frequency, to characterize the energy in the signal as a function of time and frequency. This approach has received more attention in recent years for tracking coherent turbulent structures within measurements of velocity [13-16] and wall-pressure signatures [17, 18].

The emergence of smaller, more advanced pressure transducers and full-field velocity measurement technologies, such as particle image velocimetry (PIV), have allowed for great advances in the pursuit of characterizing the organization of coherent motions in a TBL [19, 20], and inferring relationships between turbulence and wall-pressure excitation [21, 22]. However, these techniques can be intrusive, costly and complex to use, and resolution issues typically limit the streamwise and wall-normal extent of the measurement domain. As a result, flush-mounted transducers remain a popular tool for studying wall-pressure fluctuations beneath TBLs.

In this context, the general objective of this thesis is to study the characteristics of wall-pressure fluctuations beneath a fully-developed, low Reynolds number TBL, and the relationship between coherent turbulent structures and wall-pressure. Traditional statistical tools are employed to obtain a high-level understanding of the time-averaged properties of wall-pressure fluctuations. Efforts are focused towards the characterization of the energy at a single point, using the power spectrum representation, and the spatial coherence, using cross-correlation and cross-spectrum statistical descriptors. 
A novel statistical analysis is developed and presented for extracting more detailed information about the scales of turbulent motions in the boundary layer using only wavelet transformations of wall-pressure signals. The results are validated using traditional time-averaged analyses and published findings in the literature. This novel analysis has many potential applications as a complimentary tool for turbulence modeling, or developing turbulence control approaches that alter the spatial properties of wall-pressure excitations to reduce structure-borne sound.

\subsection{Literature Review}

This section presents the introductory concepts required to describe the structure of turbulence in wall-bounded flows in terms of quasi-coherent structures. These quasicoherent structures, hereforthe referred to as 'coherent structures', are commonly used for simplistic explanations of turbulence production, dissipation mechanisms, and measured characteristics of wall-pressure fluctuations. This review will provide the necessary context for in-depth discussions surrounding wall-pressure modeling efforts, and the association of wall-pressure signatures to quasi-coherent structures within the boundary layer.

\subsubsection{A review of coherent structures in wall-bounded flows}

A typical boundary layer has a number of characteristic layers that are defined on the basis of wall units, $y^{+}=y u_{\tau} / v$, which represent the wall-normal distance, $y$, normalized by friction velocity, $u_{\tau}$, and kinematic viscosity, $v$. A summary of the various regions and layers that are used to describe a TBL is provided in Table 1.1, and the layers are overlaid on a schematic of a typical mean velocity profile in Fig. 1.1 . 


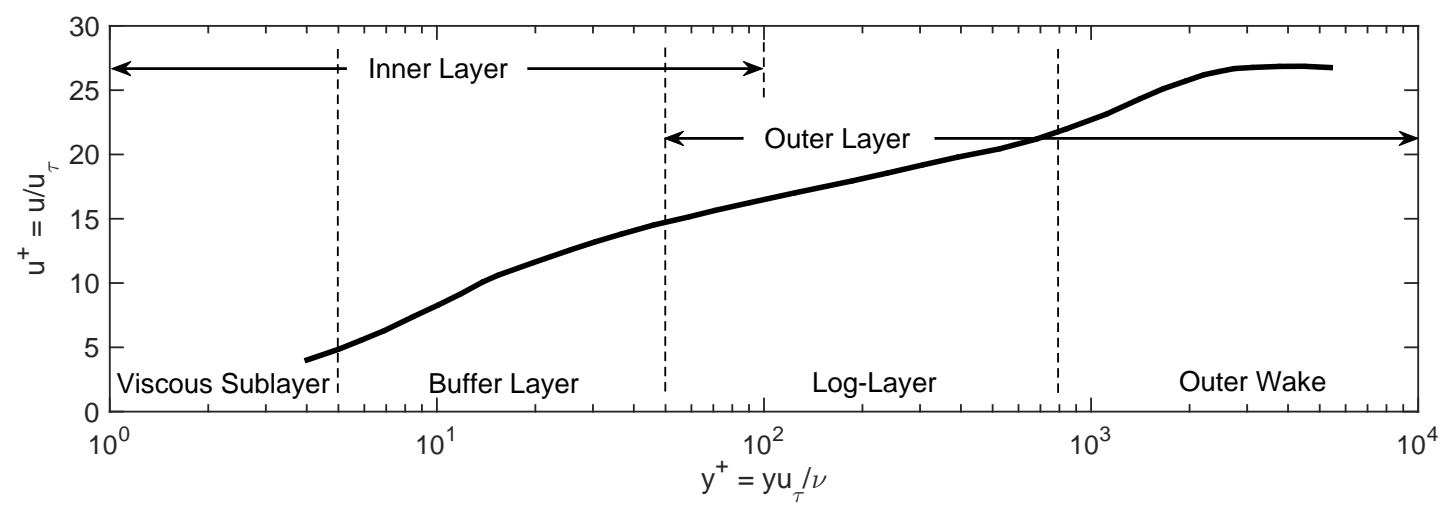

Figure 1.1: Typical mean velocity profile of a low Reynolds number turbulent boundary layer.

\begin{tabular}{|c|c|c|}
\hline Region & Location & Defining Property \\
\hline Inner Layer & $\mathrm{y} / \delta<0.1$ & Mean velocity determined by $u_{\tau}$ and $y^{+}$ \\
\hline Viscous sublayer & $y^{+}<5$ & $\begin{array}{l}\text { Reynolds shear stress is negligible } \\
\text { compared with the viscous stress }\end{array}$ \\
\hline Outer layer & $y^{+}>50$ & $\begin{array}{l}\text { Direct effects of viscosity on mean velocity } \\
\text { are negligible }\end{array}$ \\
\hline Overlap region & $y^{+}>50$ & $\begin{array}{l}\text { Overlap between inner and outer layers } \\
\text { (only visible at large Reynolds numbers) }\end{array}$ \\
\hline Log-law layer & $y^{+}>30, y / \delta<0.3$ & $\begin{array}{l}\text { Normalized mean velocity can be described } \\
\text { using a logarithmic law }\left(u^{+}=\frac{1}{0.41} \ln \left(y^{+}\right)+5.2\right)\end{array}$ \\
\hline Buffer layer & $5<y^{+}<30$ & $\begin{array}{l}\text { Region between the viscous sublayer and } \\
\text { the log-law region }\end{array}$ \\
\hline
\end{tabular}

Table 1.1: Regions and layers within a turbulent boundary layer and their defining properties (adopted from [23])

A typical mean velocity profile can be divided into an inner layer and outer layer, each with smaller regions having unique properties. The inner layer and outer layers are primarily defined based on the degree to which molecular viscosity affects shear stress and the mean velocity profile. In the inner layer these effects are dominant, 
whereas in the outer layer, shear stresses are primarily generated by turbulence interactions or Reynolds shear stress $(\rho\langle u v\rangle$, where $u$ and $v$ denote the streamwise and wallnormal components of fluctuating velocity, respectively). Not all of the regions listed in Table 1.1 may be present for lower Reynolds numbers $\left(R e_{\theta}<5,000\right)$ as some become more clearly defined only at high Reynolds numbers.

Due to the highly complex nature of wall-bounded turbulence the majority of experimental efforts to date have been focused on developing simplified, but realistic, physical models for turbulence production and dissipation mechanisms based on the concept of coherent structures. It is generally agreed upon that the internal 'structure' of turbulence consists of different coherent events, based on wall-normal distance within the boundary layer. A coherent structure is defined as a region of space, and time, that exhibits a characteristic coherent pattern [23, 24]. Experimental research has identified several categories of coherent events, which are commonly identified as follows [23, 24]:

1. low-speed streaks in the region of $0<y^{+} \leq 10$;

2. ejections of low-speed fluid outward from the wall, including lifting low-speed streaks;

3. sweeping motions of high-speed fluid towards the wall;

4. 'arch', or 'hairpin', vortices;

5. near-wall shear layers that form where high-momentum fluid opposes ejections of low-momentum fluid;

6. redirected fluid near the wall, which form 'pockets' in flow visualization measurements;

7. large-scale motions, having sizes on the order of the boundary layer thickness $\delta$; and 
8. outer-region shear layers, which form on the back of large-scale motions.

Flow visualization studies [25] have reported that the streamwise velocity field in the near-wall region $\left(y^{+}<40\right)$ consists of alternating low-speed streaks and high-speed streaks. These streaks undergo a process known as bursting where low-momentum fluid is ejected away from the wall. This process is generally constrained to the buffer layer, and is responsible for generation of the majority of Reynolds stress [24]. These streaks have been attributed to counter-rotating, quasi-streamwise vortices, which lift away from the wall in the form of a hairpin vortex [26]. Schematics of idealized, symmetric, and asymmetric, hairpin vortex structures are shown in Fig. 1.2. As the head of the vortex lifts away from the wall, the vortex becomes increasingly stretched in the streamwise direction, inducing an inrush of high-speed fluid beside the legs, and towards the wall in the downstream direction [27]. These two processes have recently been shown to be the primary mechanisms for hairpin vortex generation [27].
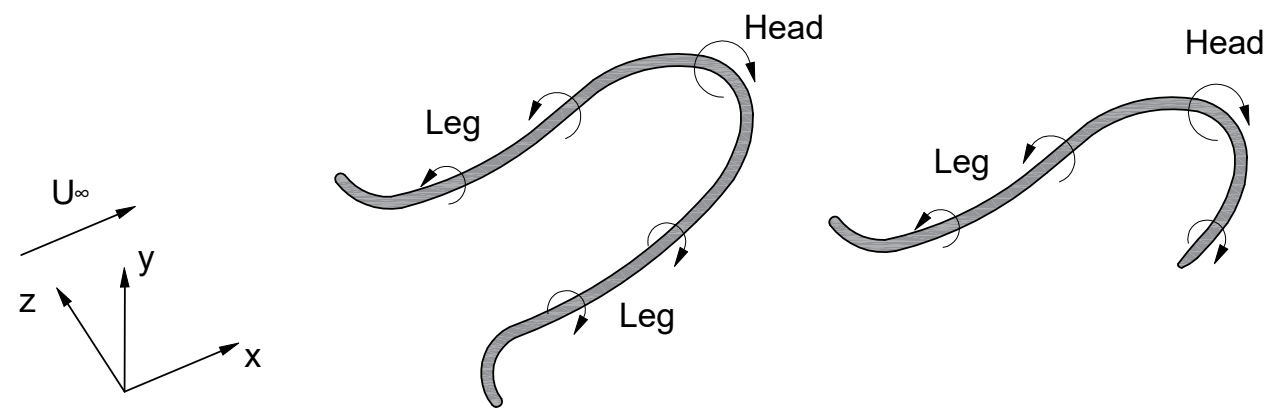

Figure 1.2: Schematic of idealistic, symmetric (left) and asymmetric (right) hairpin vortex structures. Arrows indicate the sense of circulation.

Hairpin vortices, or groups of vortices, can coalesce to form large-scale bulges, on the order of the boundary layer thickness, in the outer region. These large-scale motions can entrain pockets of non-turbulent, irrotational fluid deep into the boundary layer. These slower moving structures are opposed by higher momentum fluid near the edge 
of the boundary layer, which can form large shear layers [24].

The association of wall-pressure to non-local velocity fluctuations is difficult since wall-pressure is an integral quantity, influenced by velocity fluctuations and irrotational motions from all regions within the boundary layer. Advancements in instrumentation, and in our understanding of turbulence generation in wall-bounded flows, have led to marked improvements in the ability to model the wall-pressure field, and extract general characteristics of the boundary layer, based on measured wall-pressure fluctuations. The most pertinent efforts in the literature regarding wall-pressure modeling and studying the organization of coherent structures in TBLs, will be discussed in the following sections.

\subsubsection{Single-point frequency spectrum of wall-pressure fluctuations}

The single-point power spectrum is a common tool for studying the distribution of energy in a random signal, as a function of frequency. It has proved to be very useful for studying the physics of TBLs, and establishing relationships with wall-pressure [28]. Willmarth \& Wooldridge [7] were two of the first authors to provide a detailed account of the wall-pressure field, beneath a TBL, using the power spectrum representation. In addition, they characterized the mean-square pressure energy, and the space-time correlations of the pressure in streamwise and spanwise directions. Using the power spectrum, and the spatial dependence of the convection velocity (defined as the speed of the reference frame, in which the decay of pressure correlations is minimum), Willmarth \& Wooldridge proposed that smaller, slower, moving pressure-producing eddies decay quicker than larger, faster moving eddies. This first account of the statistical characteristics of wall-pressure fluctuations formed the foundation for more rigorous studies, and empirical modeling efforts.

Numerous accounts of the wall-pressure spectrum have since been reported. 
The absolute energy in the spectrum has shown to vary significantly with Reynolds number [7, 29, 30]; however, common features have been identified via normalization with boundary layer parameters. Normalization is based on the concept of self-similarity, in which a function of variables retains the same shape for a wide range of conditions if normalized by appropriate length, velocity and time scales [31]. As previously presented (section 1.2.1, viscous scaling is commonly applied to collapse the mean velocity profile in a TBL. The concept of normalizing the single-point frequency spectrum has been explored by several researchers [28, 29, 31-33]. However, due to the vast range of length and time scales in the boundary layer no single set of variables can collapse the entire curve for all Reynolds numbers. That said, similar to mean velocity scaling, narrowband regions of the pressure spectrum have shown to collapse on distinct combinations of boundary layer variables, which have then been used to infer the origins, and scale, of turbulent events.

In general, a typical spectrum, as shown in Fig.1.3, has four frequency ranges with distinct power law features, namely: low-frequency, mid-frequency, overlap, and highfrequency [11, 29, 31, 34, 35]. The spectral features, and normalization, of these regions will be discussed in detail, with a summary of select experimental measurements from the literature provided in Table 1.2 .

The low-frequency region contributes approximately $1 \%$ to the total root-mean square (RMS) pressure levels [29]; however, accurate modeling of these fluctuations has important implications, as low-frequency pressure fluctuations are more likely to excite the fundamental modes of the structure. Early theoretical models [44-46], and a more recent model by Smol'yakov [47], suggested an increase in low-frequency spectrum energy $\left(\omega \delta / u_{\tau}<5\right)$, proportional to $\omega^{2}$, assuming a frozen pressure field; i.e., where the 


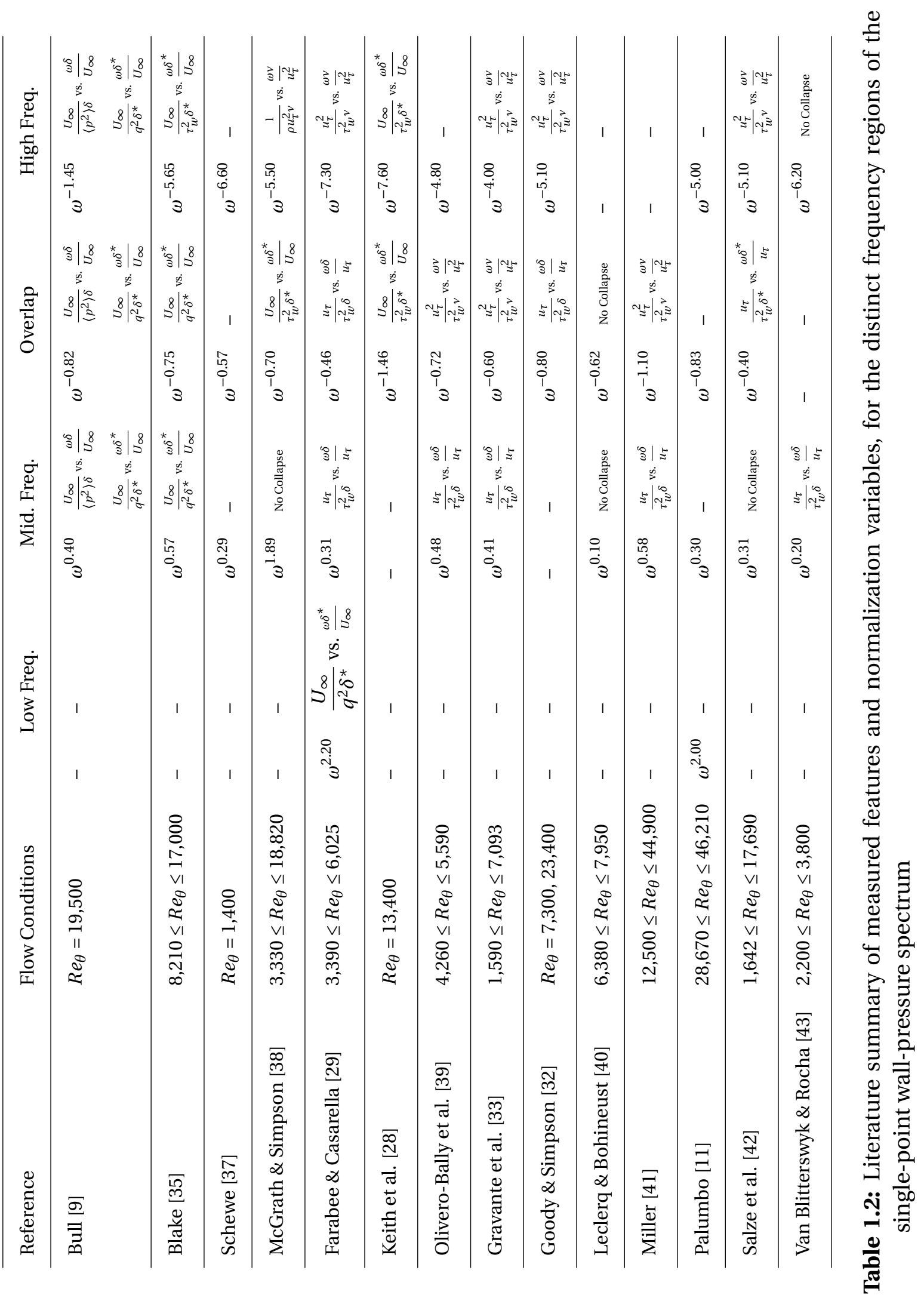




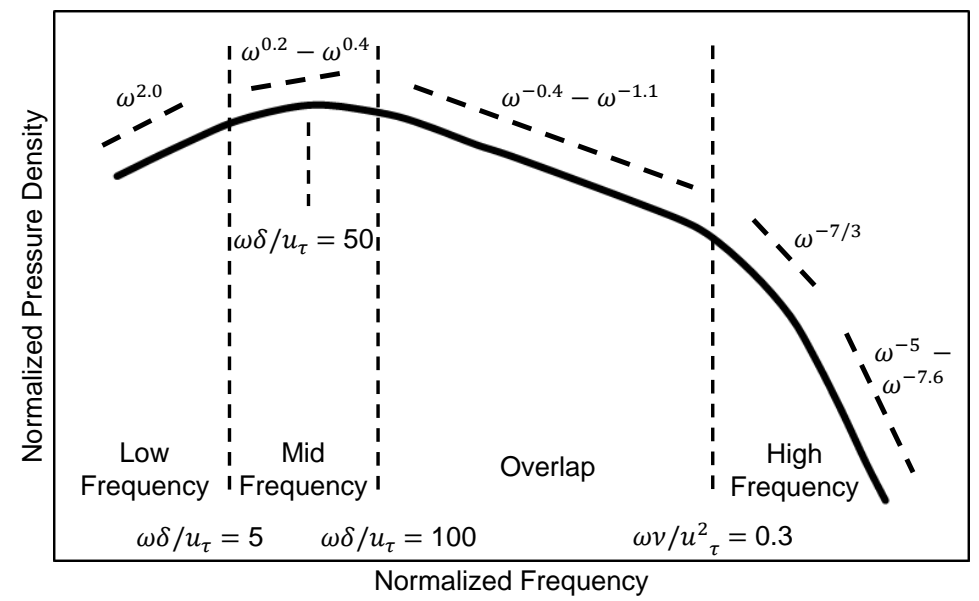

Figure 1.3: Typical wall-pressure spectrum of pressure fluctuations beneath a fully developed turbulent boundary layer (adapted from [36]).

convection speed of pressure-producing eddies is independent of their frequency. Panton and Linebarger [48] removed this assumption, and expanded the theoretical model by Kraichnan [44], which resulted in an increased energy growth rate proportional to $\omega^{1.1}$. Experimental verification of this region has proven to be difficult, due to background noise contamination [7, 9, 43, 49]; therefore, there is limited experimental evidence supporting these predicted low-frequency trends.

Early experimental measurements by Panton et al. [50], of the wall-pressure spectrum over the fuselage of a glider, showed some evidence of a $\omega^{2}$ dependence. These measurements, assumed to be absent of any contamination from background noise sources, showed a decrease in energy for frequencies below the spectrum peak $\left(10<\omega \delta / u_{\tau} \leq 50\right)$; however, instrumentation noise appeared to mask the signal where the $\omega^{2}$ trend was hypothesized to exist. With more advanced microphones and amplifiers, the measurements by Farabee \& Casarella [29], in a low-noise wind tunnel facility, and flight test measurements by Palumbo [11], are the only recent studies to show evidence of a $\omega^{2}$ energy dependence. The in-flight measurements provide a greater level of confidence that these signatures are, in fact, a physical feature of the boundary layer 
- something that could not be confirmed with wind tunnel measurements, requiring noise cancelling procedures to resolve the low-frequency response.

The low-frequency region of the measured spectra, by Farabee \& Casarella [29], collapsed well when scaled on dynamic pressure, $q$, as the pressure scale, and $\delta^{*} / U_{\infty}$, as the time scale (where $U_{\infty}$ and $\delta^{*}$ denote the freestream velocity, and displacement thickness of the boundary layer, respectively). While the implications of this scaling were not fully clarified, these variables suggest that the sources contributing to low-frequency pressure fluctuations are large in scale, and most likely associated with unsteady potential flow above the boundary layer. Keith et al. [28] and Blake [51] hypothesized that wall shear stress, $\tau_{w}$, would also be suitable for pressure scaling; however, their measurements did not extend to frequencies low enough for proper confirmation. Their hypothesis was later confirmed by Goody [31], who re-examined the scaling behaviour of several measurements from the literature, showing that the spectra collapse over a larger frequency range if pressure is scaled using $\tau_{w}$, rather than $q$. He also proposed that the boundary layer thickness, $\delta$, is preferred over $\delta^{*}$ as the length scale, since it is more representative of the scale of the largest coherent structures.

The region identified as the mid-frequency range $\left(5<\omega \delta / u_{\tau}<50\right)$ represents an intermediate growth rate in spectrum energy, between the low-frequency, $\omega^{2}$ region, up to the peak of the spectrum. This region is less defined in theoretical models [44, 48], and early empirical models [52-55], when compared to more recent empirical models [31, 47]. The increase in spectrum energy in the mid-frequency range appears to be relatively consistent between laboratory and in-flight measurements, with studies reporting a frequency dependence ranging from $\omega^{0.2}$ to $\omega^{0.4}[11,29,33,40,42,43]$. Bull [9] and Blake [35] showed that $\left\langle p^{2}\right\rangle$ and $q$ reasonably collapse the spectra; however, Goody \& Simpson [32], who reviewed the experimental spectra from several research groups, found that a combination of inner and outer layer variables, or 'mixed 
scaling, with $\tau_{w}$ as the pressure scale, was more suitable for the vast majority of studies. Literature has shown that this frequency region is insensitive to time scaling variables, with $\delta / u_{\tau}, \delta^{*} / u_{\tau}, \delta / U_{\infty}$, or $\delta^{*} / U_{\infty}$ all showing acceptable collapse (Table 1.2 [28, 29, 32, 33, 38, 39, 51]. Keith et al. [28] showed that the mid-frequency range has a more consistent collapse using mixed scaling for $\operatorname{Re}_{\theta} \leq 4,500$, and on outer layer variables at higher $R e_{\theta}$. This is characteristic of the outer layer of a TBL where the length and time scales of the outer region increases relative to the inner layer as Reynolds number increases [56, 57]. This suggests that the energy in the power spectrum over the mid-frequency range is attributed to motions in the outer regions of the boundary layer [29, 31, 39].

Theoretically, it is hypothesized that an overlap range of the spectrum should exhibit a dependence proportional to $\omega^{-1}$, beyond the peak of the spectrum [48, 51, 58]. This region is believed to exist due to its contribution from the universal, inner part of the boundary layer, where the convection velocity approaches the local mean velocity [59]. Since the convection velocity decreases as frequency increases (non-frozen pressure field), experiments have shown that the overlap region is expected to exhibit a decay closer to $\omega^{-0.7}$ [59]. The more recent model developed by Smol'yakov [47], using empirical functions for the turbulent scales, eddy viscosity and velocity defect profile, predicts a dependence of $\omega^{-1.11}$. Experimental spectra show overlap decays primarily ranging from $\omega^{-0.6}$ to $\omega^{-0.8}[9,32,35,38-40]$, which supports Bradshaw's theory of a $\omega^{-0.7}$ decay; however, experimental decays ranging from $\omega^{-0.4}$ to $\omega^{-1.1}$ have been measured (see Table 1.1). The majority of the studies with experimental decays ranging from $\omega^{-0.6}$ to $\omega^{-0.8}$ were used by Goody [31] in developing an empirical model for the single-point spectrum, for low-to-moderate Reynolds numbers, and therefore, it predicts a decay proportional to $\omega^{-0.7}$.

A unique feature of the model proposed by Smol'yakov [47] is a vanishing overlap 
region on the wall pressure spectrum for $R e_{\theta}<3,500$, which had been reported in several experimental studies on low Reynolds number flows [29, 33, 37, 38, 60]. The growth of the log-layer with Reynolds number, along with the discrepancies between inner and outer layer characteristic scales, is believed to confine the influence of the near-wall ejections to the log-layer. This allows the wake strength of the outer layer to reach an asymptotic value above $\operatorname{Re}_{\theta}>3,500$ [61]. This behaviour suggests that velocity fluctuations, contributing to the overlap region of the spectrum, originate in the log-layer [47, 58]. This is also supported by the collapse of the spectrum with mixed-scaling, as in the mid-frequency range [28, 29, 31-33, 38, 39, 51]. This Reynolds number dependence is also captured in Goody's model [31]; he proposed that the effect of Reynolds number was more aptly described using a time scale, $R_{T}=\left(\delta / U_{\infty}\right) /\left(v / u_{\tau}^{2}\right)$, representing the ratio of outer-layer-to-inner-layer timescales, rather than $R e_{\theta}$.

Empirical modeling of high-frequency pressure fluctuations is difficult due to a strong dependence on spatial attenuation, and distortion from finite-sized pressure transducers [10, 33, 62, 63]. Using pinhole-capped microphones, which reduce the effective size of the transducer, several experimental studies have reported a rapid decay in wall-pressure energy beyond the overlap range, where the spectrum transitions through a region of $\omega^{-7 / 3}[11,29,33,37]$, prior to reaching a 'final' decay rate ranging from $\omega^{-5}$ to $\omega^{-7.6}[11,28,32,33,35,38,39,42,43]$. The existence of a transition region is consistent with the properties of the buffer layer (Fig. 1.1), acting as a region between viscosity-dominated, and turbulence-dominated flow [23]. Spectrum normalization supports this theory, showing a collapse when normalized using $\tau_{w}$ as the pressure scale, and $v / u_{\tau}^{2}$ as the time scale [29, 32, 39, 42]. These inner-layer scales are equivalent to using $u_{\tau}$ as a velocity scale, and $v / u_{\tau}$ as a length scale, which are used to collapse mean velocity profiles in the viscous sublayer, buffer layer and log-layer [32]. Therefore, it is postulated that the high-frequency portion of the spectrum primarily represents the 
signature of near-wall turbulence in the buffer layer [29, 32].

Spectrum normalization has been extensively applied in the literature to study the distribution of energy in a wall-pressure signal, and to loosely associate portions of this energy to regions in the boundary layer. Normalization has also suggested that the organization of turbulent structures depends on the Reynolds number of the flow, which can have pronounced effects on the measured spectra. Accurately capturing these effects in empirical models of the spectrum has been a topic of research for the past 50 years. Several theoretical and empirical models have been proposed (please see references [36, 43, 64] for a summary); however, varying instrumentation and flow conditions among these studies has resulted in substantial uncertainty between the models.

To properly model the aerodynamic excitation induced by a TBL, one must also understand the spatial evolution of the single-point energy as it is convected with the flow. Common statistical descriptors of this behaviour have been proposed in the temporal cross-correlation, and cross-spectrum functions, which will be discussed in section 1.2.3.

\subsubsection{Spatial coherence of wall-pressure fluctuations}

In an attempt to correlate wall-pressure fluctuations to turbulence in the boundary layer, with greater resolution than that achievable using scaling variables, the spatial properties of wall-pressure signatures became the focus of several experimental studies. Common analyses include the space-time cross-correlations, or cross-spectrum correlations (also commonly known as 'coherence'), between spatially distributed signals of pressure, or pressure with velocity. Together, these studies have provided insight into time-averaged relationships between turbulent events and wall-pressure fluctuations.

Willmarth \& Wooldridge [7] showed that the cross-correlation between two microphones, separated by short distances in the streamwise direction, exhibited a local peak 
when represented against time. The cross-correlations between multiple microphones, with varying spatial separations, allowed them to examine the spatial coherent power of the wall-pressure field. Examples of typical cross-correlation curves are shown in Fig. 1.4 .

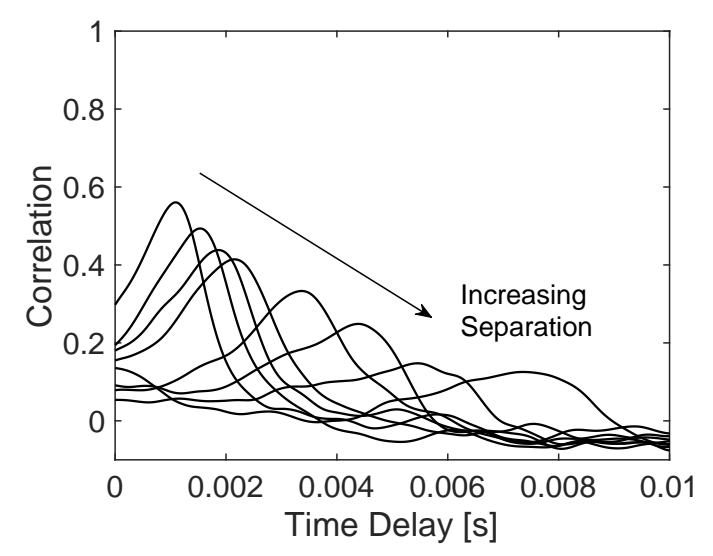

Figure 1.4: Typical cross-correlation function for microphones, with various streamwise separations, from a common reference microphone.

By plotting the peaks in cross-correlation against wavelength-normalized spatial separation $(\xi / \lambda)$, Willmarth \& Wooldridge [7 found that 'pressure-producing eddies' decayed after traveling six wavelengths downstream, with overall signal correlation persisting for $1.5 \delta$. They further utilized the time delays, corresponding to the peak in cross-correlation measurements, to define a mean 'convection' velocity of the pressure signatures. They measured a convection velocity dependence on streamwise separation and frequency, reporting variations ranging from $U_{c} / U_{\infty}=0.83$, to $U_{c} / U_{\infty}=0.69$, between low-frequency and high-frequency pressure fluctuations, respectively. Bull [9] and Blake [35] were some of the first to use pressure transducers small enough to properly capture high-frequency pressure fluctuations. Their studies confirmed the existence of the exponential decay, measured by Willmarth \& Wooldridge [7, but measured much larger decay rates. 
Bull [9] refined the spatial coherence of the pressure, by categorizing the pressure fluctuations into high-frequency and low-frequency families. On average, the highfrequency events decayed after traveling four wavelengths downstream, whereas the low-frequency events lost coherence, seemingly independently of wavelength. He also proposed that the distribution of phase velocities could be used to localize the pressureproducing events within the boundary layer, by superimposing the frequency-banded convection velocities onto the mean velocity profile. Using this approach, he postulated that the low-frequency fluctuations primarily originate in the log-layer, and highfrequency structures in the buffer layer. This approach was later applied by Schewe [37] to localize infrequent, large amplitude pressure peaks in the boundary layer, to a normalized wall distance of approximately $y^{+}=y u_{\tau} / v=21$.

Blake [35] gave special attention to the characteristics of small-scale components in the pressure field and showed that, at very small separations, the broad-band convection receives significant contributions from the buffer layer, especially when considering correlations transverse to the direction of the flow. This influence was supported by the better collapse of streamwise space-time correlations, when normalized using inner-layer scaling, $v / u_{\tau}$, compared to $\delta^{*}$ (as used by Willmarth \& Wooldridge [7]).

Thomas \& Bull [65] studied the temporal correlations between low-frequency and high-frequency pressure fluctuations, and concluded that high amplitude, highfrequency pressure fluctuations could be associated with a low-frequency pressure transition from a large, positive value to a large, negative value. Both low-frequency and high-frequency pressure fluctuations were found to coincide with peaks in wall shear stress; however, this did not provide sufficient evidence to associate these pressure events with turbulent structures.

Palumbo [11] later conducted a similar investigation, to isolate large-amplitude coherent events within pressure signatures, using a 'convected' cross-correlation 
analysis. Phase-shifting the time series by the estimated convection velocity revealed that coherent events maintain higher levels of correlation over their life. Based on this, Palumbo suggested that the rapid decay in broadband correlation, with increasing streamwise separation (see Fig. 1.4), is not representative of individual events, but of the distribution of correlation behaviours for all events contributing to the wall-pressure signature.

Similar to the correlation length, the spatial energy of the wall-pressure can be described using a 'coherence length', defined from the spatial decay rate of the crossspectrum between two microphones. It is well accepted that the cross-spectrum amplitude is dependent on spatial separation, with the peak coherence occurring near the peak frequency of power spectrum, and rapidly decaying at higher frequencies, in a near exponential manner [10]. The loss of coherence at high frequencies indicates that these pressure fluctuations are destroyed rapidly as they are convected. It is thought that the signature of these fluctuations are representative of turbulent motions originating near the wall, or short-lived motions in the outer layer [29, 40]. At frequencies above the cross-spectrum peak, the coherence collapses to an exponential form, when normalized by the spatial separation, $\xi$, and mean convection velocity, $U_{c}$, referred to by Cor$\cos [10]$ as 'similarity scaling'. This indicates that these pressure fluctuations decay at an equal rate, in convecting equal distances downstream proportional to their wavelength [29]. Spanwise cross-correlations exhibit similar features, but decay by almost an order of magnitude more rapidly, with increasing spatial separation [3, 9, 11, 29, 40, 42, 66]. Blake [35] proposed that any correlations in the spanwise direction are a result of upstream disturbances, spawning from presumed outer-layer, low-frequency eddies.

At low frequencies, recent studies [3, 11, 29, 38, 40, 66] have provided evidence that invalidates the use of a coherence length scale, showing approximately constant coherence levels that are well below unity, and coherence lengths that decrease with 
decreasing frequency. This follows from consideration that the boundary layer thickness and shear stresses, limit the scale and lifetime of the physical processes, which generate the pressure fluctuations. These low-frequency signatures also decay much slower than predicted when using the common exponential function for coherent power decay [10].

The exact extent of this coherence is still debated, with recent measurements reporting sustained coherence up to streamwise separations, ranging from $4 \delta$ to $7 \delta$ [11, 29, 40, 50, 67]. This extended coherence was commonly associated with large, irrotational motions outside of the boundary layer [29,40]. However, pressure measurements alone prohibit definitive association of these pressures to turbulent structures. This conjecture was supported by Wilczynski \& Casarella [68], who measured elevated coherence between wall-pressure and simulataneous measurements of velocity near the outer regions of the boundary layer. There is also supporting research that other well-defined events, such as the formation of hairpin packets, could contribute to the extended streamwise coherence [19, 20, 69]. These structures consist of several smaller coherent structures, creating an extended region of low-momentum fluid, which is convected for large distances downstream.

\subsubsection{Identifying coherent structures in the turbulent boundary layer}

Standard time-averaged correlation techniques, and Fourier-based coherence analyses, play an important role in understanding the aggregate characteristics of the wall-pressure signatures. Temporal correlations, however, provide no information about the space scale, and Fourier-based approaches lose all frequency locality in the signal, making it difficult to relate characteristics of the spectrum, to physical features in the flow [12]. For this reason, conditional averaging techniques have been applied with reasonable success $[65,68,70-76]$, to extract features of high amplitude pressure peaks, or 'HAPPs'. HAPP signatures occur infrequently (less than $10 \%$ of the time [70]) 
but contribute up to $60 \%$ of the RMS pressure [22], and have time scales that span the low-frequency to mid-frequency ranges of the wall-pressure spectrum, suggesting that many turbulent events are linked to their occurrence [68, 73, 74].

Conditional averaging is the process of averaging short segments of time surrounding an identified event, or 'trigger event', which is generally defined as a peak in pressure that exceeds some multiple of the RMS pressure level. This approach was used by Thomas \& Bull [65] to confirm results from temporal correlations, relating high-frequency pressure peaks with large, low-frequency, positive-to-negative pressure fluctuations, using high-frequency pressure peaks as the trigger event. They also proposed that the HAPPs are related to a 'bursting' pattern in streamwise velocity fluctuations $(u)$, whereby the velocity fluctuations exhibit a deceleration, followed by a rapid acceleration, and a slow return to the average value. From this, they concluded that the high-pressure patterns convect with large, inclined shear layers, which are formed on the upstream side of the burst event. These events can traverse most of the boundary layer, with a convection velocity of approximately $0.67 U_{\infty}[65]$. Interestingly, these structures did not convect at the same speed as the pressure signatures correlated at large streamwise distances, and it was therefore proposed that those correlated signatures are related to large-scale motions in the outer region of the boundary layer [65, 68, 71].

Guezennec [75] corroborated these results, and also detected two smaller, and more broad, pressure peaks following a large, negative pressure peak, by using Reynolds stress for event detection, rather than strictly streamwise velocity. He also associated large, positive HAPPs with two consecutive turbulence-producing events in opposite directions (sweep-ejection). The two-scale character of the wall-pressure fluctuations was further supported by Kobayashi [71], who showed that burst events initiate from paired streamwise vortices, which appear in the periodic large-scale motions. 
Johansson, Her \& Haritonidis [70] later further developed the conditional averaging technique, into the well-known variable-interval time-averaging (VITA) technique, and added wall-normal velocity measurements to corroborate some of the findings of Thomas \& Bull [65], and Guezennec [75]. The large, positive pressure peaks were mainly found in isolation, or with some smaller pressure peaks occurring less than $10 \%$ of the time. A reduced correlation beyond the buffer region suggested that shear layers were primarily constrained to the inner layer, which contradicted the results from Thomas \& Bull [65]. They also proposed that the negative HAPPs occurred during the deceleration of the sweep event, however, this relationship was not found to be as strong as that for the positive pressure peaks. Regardless, all studies confirm that, during HAPP events, the pressure peak is more closely related with the velocity gradient, than the velocity itself, as first proposed by Bradshaw [59]. Both high-frequency and low-frequency events, identified by Thomas \& Bull [65], contribute to the pressure patterns measured at the wall, as shown by a 'double-hump' cross-spectrum $(\gamma)$ between pressure and velocity within the buffer layer (Fig. 1.5$][68,74]$. This was not directly evident from earlier studies, which showed a decreasing correlation between pressure and streamwise velocity fluctuations, with increasing distance above the buffer layer [65, 70, 76]. Snarski \& Lupetow [74 measured a notable phase offset between wall-pressure and velocity, both near the wall, and in the outer regions of the boundary layer, due to the inclined nature of the shear layer; a likely explanation for the incoherence measured above the buffer layer by the previous studies.

Literature shows that point measurements can be interpreted in slightly different ways, resulting in discrepancies in the physical models, linking velocity and wallpressure. As fluid measurement technology developed, hotwire probes began to fall out of favour to PIV, which became more commonly used in conjunction with wall-pressure 


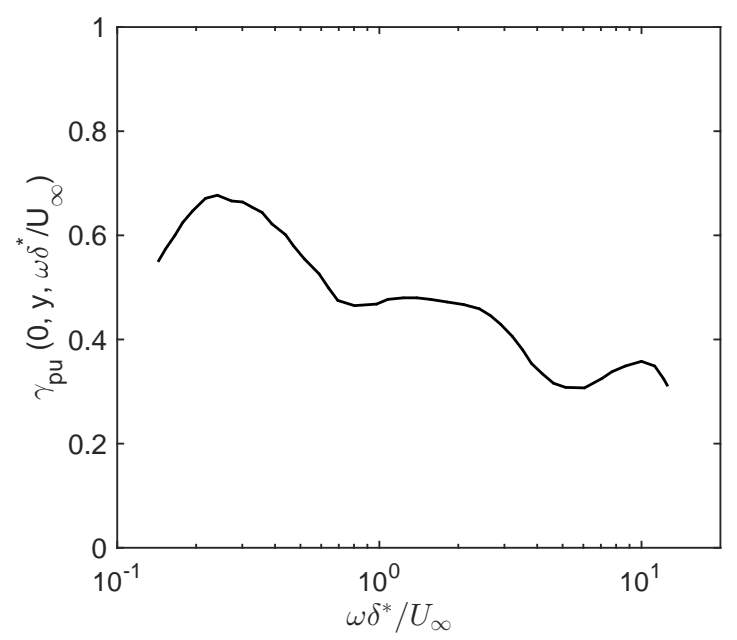

Figure 1.5: Cross-spectrum function between velocity in the buffer layer $\left(y^{+}=14(y / \delta=\right.$ 0.016)), and wall-pressure as measured by Snarksi \& Lupetow [74].

microphones, to develop the understanding of relationships between wall-pressure and coherent turbulent motions. The findings from the most pertinent studies regarding the identification and characterization of coherent turbulent structures in TBLs, and established relationships with wall-pressure fluctuations, will be discussed in the following paragraphs.

De Ojeda \& Wark [77] utilized two-dimensional PIV to resolve the velocity field between the buffer layer, and up to $2 \delta$ in the wall-normal direction, in combination with a microphone array, which spanned $\delta$ in the streamwise direction. Using spacetime correlations, they found that large, negative pressure events are accompanied by smaller, positive pressure events downstream, prior to detection, and large, positive peaks are accompanied by smaller, negative peaks upstream, similar to that measured by Guezennec [75], and Johansson et al. [70]. However, they were able to, more specifically, associate two positive HAPPs with spanwise vorticity, and the stagnation point between high and low-speed fluid at the shear layer interface. The negative pressures were associated with two different velocity events. The first was associated with the 
formation of an eddy, with spanwise vorticity that ejects fluid from the wall, and induces a negative pressure. The second negative pressure event was typically found beneath a structure rotating in the opposite sense of the mean spanwise vorticity, or an 'inverse shear layer', located downstream of a large shear layer. The use of PIV allowed them to detect up to six successive eddies along the shear layer, which could span the entire boundary layer. These consecutive eddies suggest that hairpin vortices occur in groups, or 'packets', where one rides on the back of others. These structures were found to convect between $0.50-0.70 U_{\infty}$, with occassional larger structures, convecting at $0.35 U_{\infty}$, which is in agreement with the shear layer convection measured by Thomas \& Bull [65]. The finding of multiple eddies, on the back of a shear layer, emphasizes a key limitation of the VITA approach, in that typically only one event is identified, due to the necessary high threshold for reliable detection. The negative and positive pressures were commonly separated by 125 viscous units $\left(x^{+}=x u_{\tau} / v\right)$ in the streamwise direction, and closely related with the passing of eddies, which suggests that wall-pressure can be used to identify distinct hairpin structures.

Adrian et al. [19] confirmed that the HAPPs can be explained by the passing of a hairpin packet. These packets include several individual hairpins, that are aligned in the streamwise direction (Fig. 1.6), and convect at a speed of approximately $0.8 U_{\infty}$. The nesting of these smaller hairpins creates the appearance of persistent, uniform streamwise momentum, which retains its coherence, over several boundary layer thicknesses, in the streamwise direction [19, 69]. Adrian et al. [19] also applied the VITA, and quadrant methods of conditional averaging, to the velocity fields to illustrate the physics behind the detected events (Fig. 1.6). The quadrant method is similar to the VITA approach, with the exception that it considers the temporal polarity of both streamwise, and wall-normal velocity fluctuations, for event detection and classification. Adrian et 
al. [19] detected a vast range of scales within the hairpin packets, as shown by the occurrence of numerous distinct events in different frequency bands. This supported the findings of Wilczynski \& Casarella [68], Snarski \& Lupetow [74] and Karangalen et al. [73]. These packets are now believed to be the most likely explanation for the extended coherence measured using the traditional time-averaged cross-correlation and Fourier-based coherence functions.

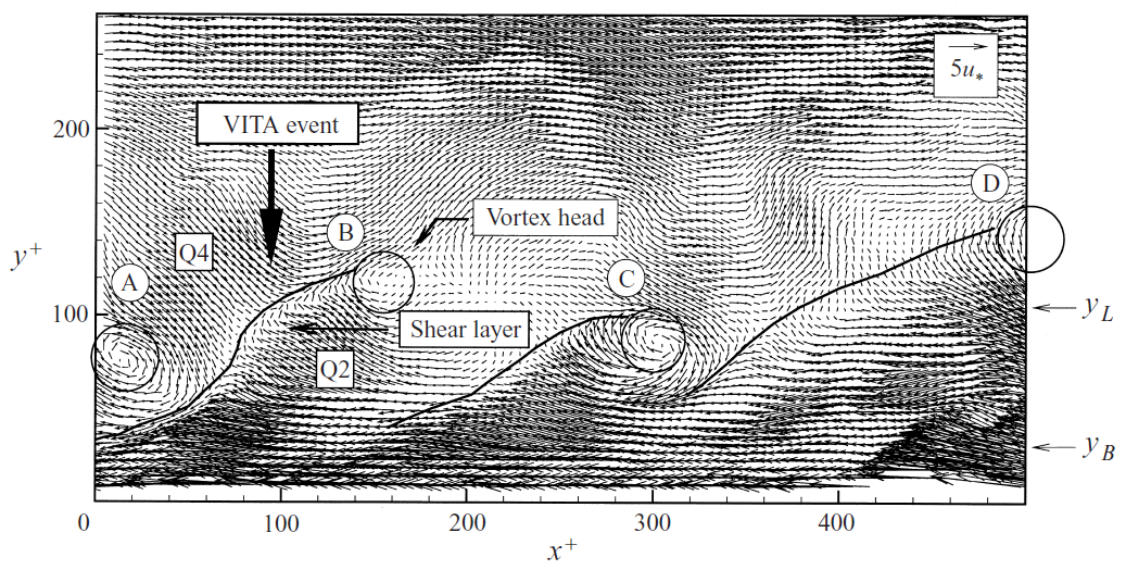

Figure 1.6: The identification of four hairpins, aligned in a streamwise packet, as measured by Adrian et al. [19], using two-dimensional PIV. The velocity field is presented in a frame of reference moving at $0.8 U_{\infty}$. ' $y_{L}$ ' and ' $y_{B}$ ' denote the extents of the log-layer and buffer layer, respectively, and 'Q2' and 'Q4' denote classifications of events detected using the quadrant method.

Ganapathisubramani et al. [20] performed a detailed study, using PIV on hairpin packets, previously identified by De Ojeda \& Wark [77] and Adrian et al. [19]. The velocity fields showed signatures of vortex packets in the log-layer, with groups of legs found to be coherently aligned in the streamwise direction. This organization was found to breakdown outside of the log-layer, however, Marusic [78] suggested that packets of hairpins do not need to extend above the log-region to retain extended streamwise coherence.

In a later study, Ganapathisubramani et al. [79] used two-point correlations to infer more properties about these hairpin packets. Correlations of wall-normal velocity 
were found to occur in discrete, but consecutive zones, extending over large streamwise distances, as would occur from the upwash induced between the hairpin legs in a packet. In the log-layer, these correlations are shorter in the streamwise direction compared to the spanwise direction, and may explain the 'upstream disturbances' responsible for spanwise pressure correlations, as discussed by Blake [35]. Most packets of elevated vorticity were observed with up to three hairpins, separated by 100-150 wall units in the streamwise direction, confirming the spacings measured by De Ojeda \& Wark [77]. Further from the wall $\left(y^{+}=198\right.$ - 503), a series of coherent structures were identified but no pattern of low-speed streaks, or vorticity, in the train of hairpin packets was observed. This suggested that the legs do not extend past $y^{+}>198$, and that only the head is identified as a coherent structure. Ganapathisubramani et al. [79] reported cases of streamwise coherence up to $2 \delta$, which was in good agreement with the measurements by Tomkins \& Adrian [80] (up to 2.5 $)$ ), and Townsend [81] (up to $3 \delta$ ); however, this coincided with the streamwise extent of the PIV illumination domain.

Numerical simulations have been applied sparingly due to high computational expenses, however, Lee \& Sung [82] attempted to characterize the spatial extent of hairpin packets using the same feature extraction technique [20], on a direct numerical simulation of a turbulent boundary layer. They reported hairpin packets in the loglayer, with lengths up to $3 \delta$ - $4 \delta$, with the overall large-scale motion retaining statistical coherence up to $6 \delta$, which is in good agreement with the extent of cross-correlation and cross-spectrum energy in experimental wall-pressure measurements [11, 29, 40, 50, 67].

Ghaemi \& Scarano [22] and Ghaemi et al. [21] used three-dimensional and two-dimensional PIV measurements, respectively, in conjunction with wall-pressure measurements from a flush-mounted transducer, to study the relationship between wall-pressure and turbulent motions within the boundary layer. Ultimately, no definitive correspondence could be made between HAPPs and a specific arrangement of 
the low-speed and high-speed streaks [22]; however, using instantaneous realizations, Ghaemi \& Scarano [22] made several useful observations. Negative HAPPs appeared to be located within the core of a quasi-streamwise vortex, with positive pressure fluctuations observed in the upstream and downstream locations of the negative HAPP (Fig. 1.7).

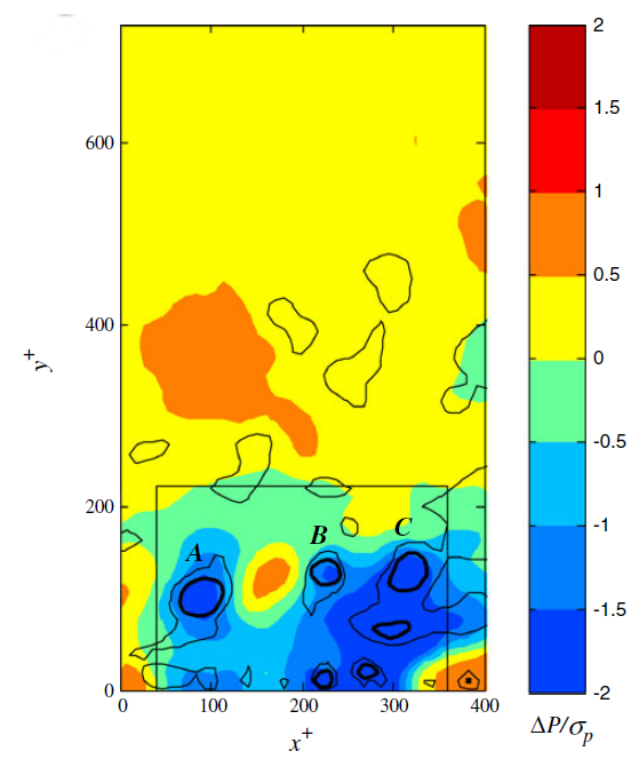

(a)

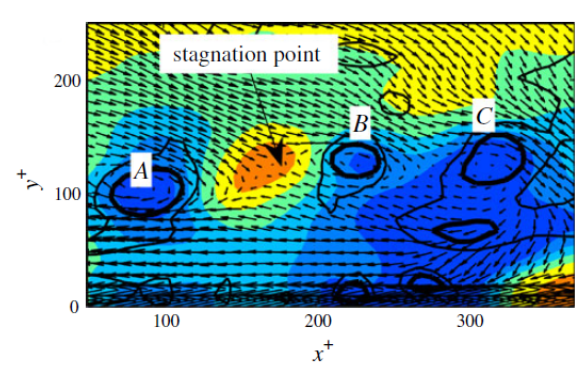

(b)

Figure 1.7: The instantaneous pressure field in a turbulent boundary layer from Ghaemi et al. [21]: a) full extent of the measurement domain, and b) a magnified view of the identified vortices near the wall. The labels ' $\mathrm{A}$ ', ' $\mathrm{B}$ ', and ' $\mathrm{C}$ ' represent three vortex heads identified from the velocity field (vector lines shown in b), relative to a convecting frame of reference at $0.78 U_{\infty}$ ).

The positive HAPPs were typically surrounded by vortical structures. The identified structures eject low-speed fluid into the outer region of the boundary layer, which is opposed by induced flow from the upstream vortex, creating a shear layer [21, 22]. The positive HAPP occurred under the stagnation point, on the upstream side of the shear layer, beneath decelerating high-speed fluid, as reported by De Ojeda \& Wark [77]. Previous studies had suggested that negative HAPPs were associated with large-scale 
sweep events, but Ghaemi \& Scarano [22] concluded this was not the source of the HAPP, as large-scale motions from the outer layer covered two positive pressure fluctuations. Finally, it was proposed that successive HAPPs (alternating positive then negative) occur as the recovery to previous events, with acceleration region of the negative HAPP acting as the recovery region for the upstream positive HAPP, and vice versa.

PIV systems and numerical simulations have been instrumental in advancing the understanding of coherent structures and their organization within a TBL, and establishing some fundamental relationships with wall-pressure; however, a review of the literature shows that a complete understanding of these relationships have not yet been clarified. This is in part due to some limitations of existing instrumentation. PIV systems require a critical trade-off between resolution and illumination volume, which typically hinders characterization of the full spatial coherence of large-scale turbulent structures. PIV systems also suffer from measurement noise and tend to attenuate the amplitude of pressure fluctuations [22]. Numerical simulations are computationally expensive, especially for studying Reynolds numbers of relevance to transportation vehicles.

Therefore, it is believed that wall-pressure measurements, obtained with flushmounted microphones, may compliment measurements from PIV or hotwire sensors to study the relationships between wall-pressure and turbulence. Wall-pressure microphones are non-intrusive, enabling the convection of coherent motions to be tracked, which would be far more difficult with instruments such as hotwire sensors. The spatial resolution of wall-pressure measurements, with flush-mounted transducers, is highly customizable, and can exceed that of PIV domains, without compromise in frequency resolution. The high-sensitivity of these transducers, over a large, dynamic frequency range, offers potential to serve as a supplementary technique for studying the organization and convection of smaller-scale structures, which contribute to persistent pressure 
signatures. If more definitive relationships between wall-pressure and coherent structures could be established, microphone arrays may also be used to develop active control strategies for near-wall turbulence [83]. Microphones may also be implemented for in-service studies, whereas PIV and hotwire sensors are generally restricted to laboratory environments.

While wall-pressure arrays cannot outright replace other instruments for turbulence studies, they have shown good potential for studying properties of large-scale coherent structures, and simultaneously establishing a better understanding of their relationships with the wall-pressure field [17, 18]. However, a suitable processing technique has yet to be developed for wall-pressure measurements that enables sufficient resolution for studying the multi-scale nature of coherent motions.

\subsection{Objectives of the Thesis}

This thesis aims to use statistical analyses of wall-pressure measurements to study the organization of the coherent structures in a TBL, and characterize their streamwise coherence using arrays of aligned microphones. More specifically, the efforts include:

1. Evaluation of the accuracy of existing single-point models, through comparison against experimental spectra. This was an objective of a preliminary study [43], however, the flow conditions did not permit proper evaluation under fully developed boundary layer conditions. Since that study, several modifications were made to the facility to improve the flow conditions, which will be discussed in this thesis.

2. Characterization of the spatial coherence of wall-pressure fluctuations, using traditional space-time cross-correlations, and Fourier-based cross-spectrum analyses, with comparisons made to measurements in the literature. 
3. Investigation of the level of energy contributed to the wall-pressure spectrum, from velocity fluctuations in various wall-normal positions within the boundary layer, using a cross-spectrum analysis between wall-pressure and velocity fluctuations.

4. Exploration of two techniques, for processing wavelet transformations of wallpressure measurements, to identify coherent events in the boundary layer. In the first approach, a wavelet cross-correlation, defined using wavelet transforms from two microphones, is used to extract events based on a correlation amplitude threshold. Previous studies have shown that the multi-scale nature of large-scale structures may be extracted using the kurtosis (intermittency) of measured velocity signals $[14,15,84]$; however, the relationships have yet to be fully clarified, and to the authors knowledge, this approach has also yet to be studied using wallpressure fluctuations. Therefore, the second approach utilizes the intermittency of energy in the wavelet coefficients to identify periods in the wall-pressure signal with high activity. The kurtosis is computed over all scales, and over discrete frequency bands to further study the organization of smaller events contributing to the large-scale periods of high activity. Features of the wall-pressure fluctuations during the high activity periods are also used to infer characteristics of individual hairpins, and hairpin packets, such as: spatial arrangement, length, convection velocity, and spatial coherence. These analyses will be used to provide insight into the statistical properties of wall-pressure fluctuations obtained using traditional time-averaged analyses. 


\subsection{Structure of the Thesis}

Chapter 1 provided a detailed overview of the most pertinent literature regarding the modeling of the wall-pressure energy at a single point, and the spatial coherence of this energy. Further, the state of the art in identifying coherent structures, in wall-bounded flows, is summarized along with measured relationships between coherent structures and wall-pressure signatures.

Chapter 2 describes the experimental facility, instrumented test panels, hotwire anemometry system, design of the hotwire probe traverse, microphone calibration and noise filtering approaches, and the procedure used to select the boundary layer tripping system.

Chapter 3 presents the statistical theory used to characterize wall-pressure fluctuations along with empirical models for the single-point wall-pressure spectrum, convection velocity, and streamwise cross-spectrum.

The results from statistical analyses of wall-pressure measurements are presented and discussed in Chapter 4. The single-point frequency spectrum, cross-correlations, convection velocity, cross-spectra, and coherence lengths are all presented first. These traditional analyses are used to establish a fundamental understanding of wall-pressure fluctuations and its relation to coherent motions in the boundary layer. The novel approach for identifying coherent structures within wall-pressure signatures, using the intermittency of wavelet coefficient energy, is demonstrated on a sample ensemble of pressure. The statistics are interpreted using findings from the literature, and used to study the length, convection speed and multi-scale composition of large-scale coherent motions.

A summary of the results, and recommendations for future work is provided in Chapter 5 . 


\section{Chapter 2}

\section{Experimental Setup and Instrumentation}

This chapter describes the experimental facility in which the experiments were performed, including: modifications made to the original test section, the selection procedure for the boundary layer trip, the integrated hotwire anemometry system for mean flow characterization, and the microphone-instrumented test panels. Information on data acquisition and calibration methodologies are also provided.

\subsection{Experimental Facilities}

The experiments were conducted in the subsonic, closed-return wind tunnel facility at Carleton University. The airflow is provided by a fan with an outer diameter of $1.19 \mathrm{~m}$, which is driven by a $37.3 \mathrm{~kW}(50 \mathrm{HP}) \mathrm{DC}$ motor that operates at speeds up to $900 \mathrm{RPM}$. A variable frequency drive (VFD) modulates the rotational frequency of the fan at a resolution of $1.0 \mathrm{~Hz}$, and a pitot-static probe, located just downstream of the inlet, was used to calibrate the linear relationship between motor frequency and wind tunnel velocity. From the calibration it was determined that the VFD could control the flow speed in increments of $0.9 \mathrm{~ms}^{-1}$, up to a maximum speed of approximately $45 \mathrm{~ms}^{-1}$. A series of turbulence grids precede a 10:1 contraction, which reduced the turbulence intensity 
levels in the center of the test section to less than $0.27 \%$, as measured for speeds up to 15 $\mathrm{ms}^{-1}$. The tunnel has a removable, rectangular test section measuring $1.83 \mathrm{~m}$ in length and $0.76 \mathrm{~m} \mathrm{x} 0.51 \mathrm{~m}$ in width and height, respectively. $3 \mathrm{~mm}$ trip wires were installed at $30 \mathrm{~mm}$ and $20 \mathrm{~mm}$ upstream of the inlet to promote boundary layer transition.

\subsection{Test Section Design and Modifications}

The test section was constructed out of 0.75 inch plywood with a small plexiglass viewing window near the back of the test section, and a small access door on the side wall to permit access to the $0.55 \mathrm{~m}$ long, $0.45 \mathrm{~m}$ wide, flush-mounted test panel within the floor of the test section. The test section is formed by two separate pieces. At the onset of the work, the ceiling and sides of the test section were defined by a large acoustically treated C-channel, with a 1 inch-thick wedged foam lining. The side walls fit over a lower chamber, $1.52 \mathrm{~m}$ in length, to form the floor. In this configuration the leading edge of the test panel was located $0.49 \mathrm{~m}$ downstream of the inlet, and a $0.31 \mathrm{~m}$ gap was left in the floor just upstream of the diffuser. A schematic of the assembled original test section is shown in Fig. 2.1.
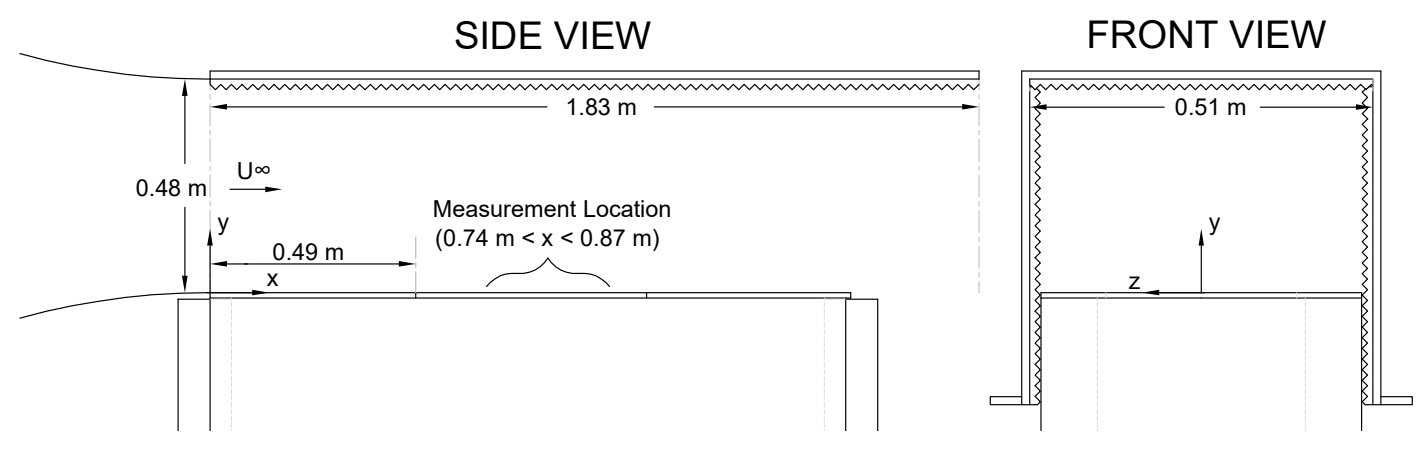

Figure 2.1: Diagram of wind tunnel test section in its initial configuration.

To accommodate installation, the downstream end was designed with an adjustable collar (Fig. 2.2), which enabled the test section to be shortened when being moved into 


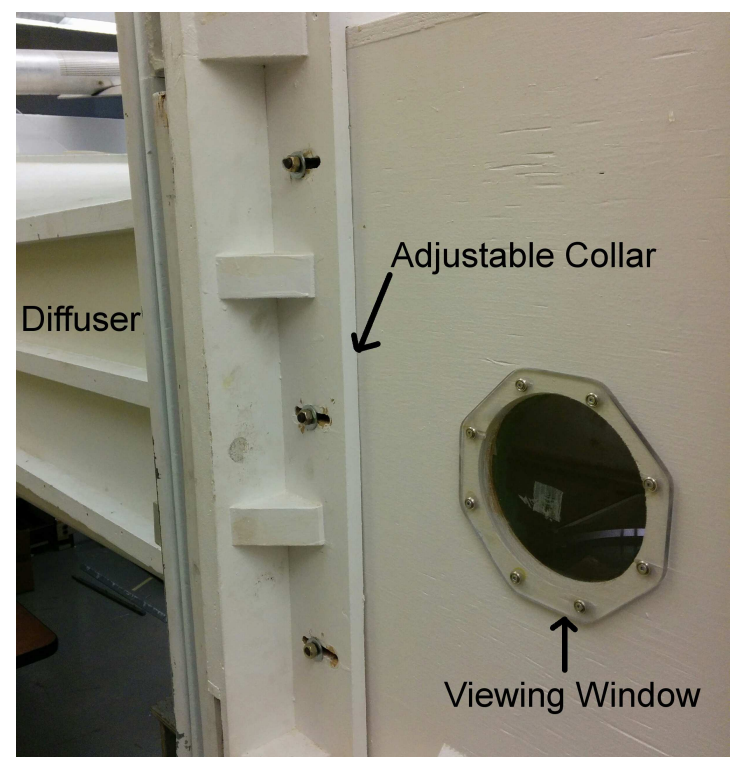

Figure 2.2: Adjustable collar used to facilitate installation of the test section within the wind tunnel circuit.

place, and then extended to mate with the face plate of the diffuser. The collar could be tightened in place using 10 bolts, around the periphery of the test section, with the overall section clamped to the face plates of the inlet and diffuser.

The lower section, which formed the floor, sat on a large platform with adjustable legs to ensure the floor was level and flush with the inlet. The lower chamber was acoustically treated to facilitate later stages of research focussed on sound radiation from thin panels subjected to boundary layer excitation. The chamber, forming the floor of the test section, was of composite construction, consisting of a 1/8 inch sheet of aluminum, bonded to $1 / 2$ inch-thick MDF board.

The immobile nature of the assembled test section was not conducive for short turnaround times between experiments requiring different test sections. Therefore, a support cart was designed and constructed to allow the entire assembly to be easily rolled in and out of place in the tunnel (Fig. 2.3. The rectangular base frame was outfitted 
with four smaller platforms in each corner, on which lab jacks were mounted to support the lower section. This eliminated the previous large and heavy base platform, but maintained the capability to carefully control the position of the lower section relative to the inlet. To support the upper section, a second rectangular frame was offset from the lower frame by four support legs ('Upper Frame' in Fig. 2.3). Two angle brackets were welded on the outside of the longer sides of this frame to support the wide, lower edge of the upper section (see front view in Fig. 2.1). Holes were drilled along this channel to bolt the upper section to the frame.

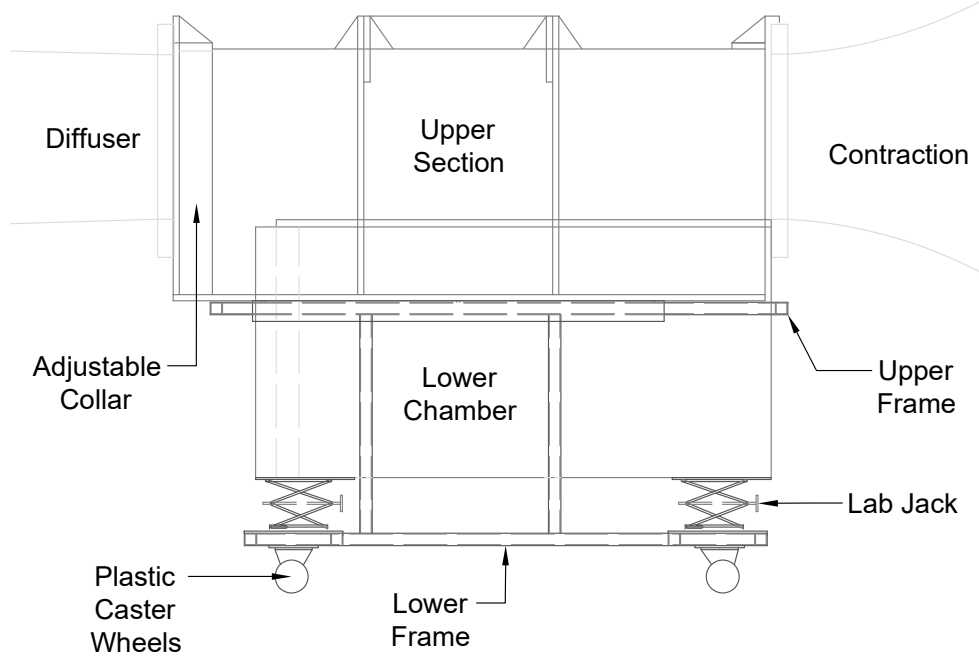

Figure 2.3: Schematic of assembled test section with new support cart (bold).

\subsubsection{Design of the adjustable ceiling}

The foam-covered interior walls of the test section caused rapid boundary layer growth and a moderate streamwise favourable pressure gradient. To compensate for boundary layer growth on the floor and ceiling, a flexible ceiling was designed, manufactured 
and installed. To avoid permanent modifications to the test section structure, the ceiling and supports were designed to be removable and self-contained on a two-track rail system. A continuous sheet of semi-flexible, 1/8 inch-thick MDF particle board was suspended from the ceiling using eight sets of evenly-spaced $(\Delta x=203 \mathrm{~mm})$ attachments. Each set was connected by a $1 / 4$ inch-diameter, aluminum rod that spanned the width of the test section to stiffen the ceiling (Fig. 2.4a). The ability to adjust the ceiling at eight streamwise locations provided a good compromise between load distribution and profile resolution. To maintain alignment during installation, and to increase the rigidity of the ceiling support system, the supports were interconnected using an aluminum frame (Fig. 2.4b).

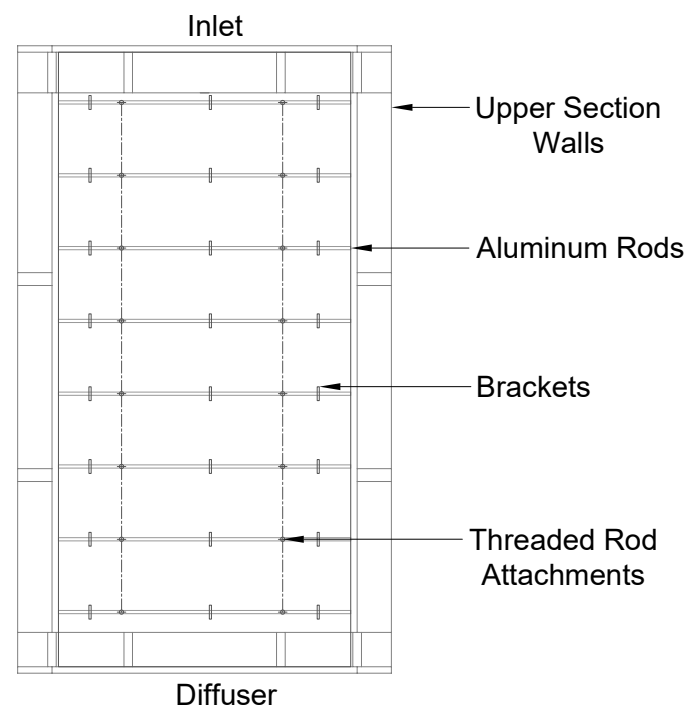

(a)

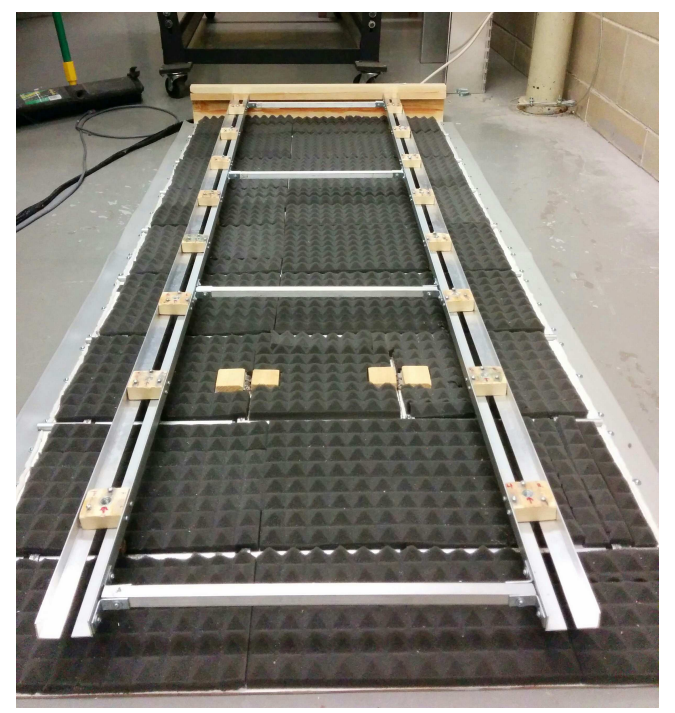

(b)

Figure 2.4: Flexible ceiling designed for the test section: a) top view schematic of ceiling support layout and, b) completed ceiling structure prior to installation.

The ceiling supports were made adjustable using a threaded rod assembly, as shown schematically in Fig. 2.5. To permit relative rotation of between the aluminum bars and threaded rods, compression fit couplings were permanently bonded to the ends of the threaded rods. To interface the two, a nut was bonded to the end of the threaded 
rod, which was then bonded to the coupling. Bonded to the compression fit insert was a threaded insert, within which a flat head screw was bonded. The head of the screw formed the interface between the aluminum bars and the ceiling support. The threaded rods were supported on the other end by recessed, threaded coupling nuts within support blocks. The blocks were outfitted with pegs to enable the supports to slide within the rails. Each support provided 35-50 mm of vertical travel. The ceiling was slotted over $150 \mathrm{~mm}$, between the sixth and seventh streamwise attachment points $(x=1.22 \mathrm{~m}$ to $1.42 \mathrm{~m}$ ) from the inlet, to permit streamwise traversing of a hotwire sensor. Flexible rubber skirts were also installed along the edges of the flexible ceiling and along the traverse opening to minimize air leakage. The upper section was necessarily raised to accommodate the ceiling support system, which sat approximately $9 \mathrm{~cm}$ below the original ceiling. The ceiling construction permits zero-pressure-gradient, mild favourable-pressure-gradient, or strong, un-sustained pressure gradient conditions to be imposed on the flow.

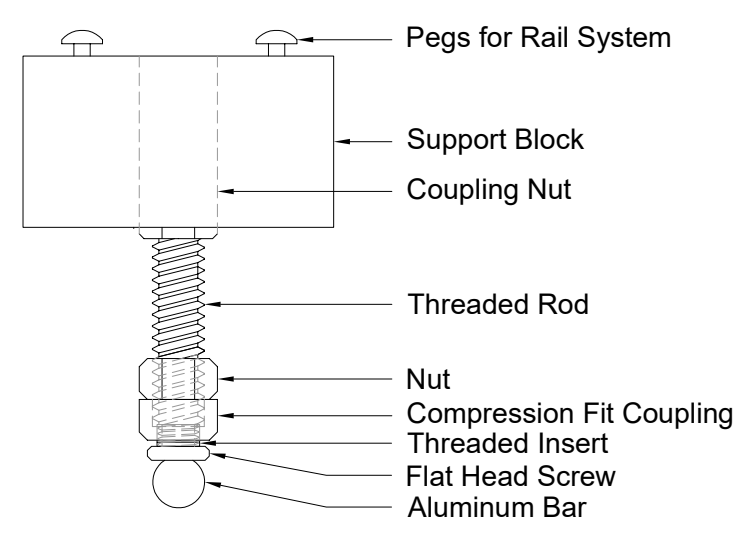

Figure 2.5: Schematic assembly of an adjustable ceiling support.

After installation, the streamwise pressure gradient was estimated using velocity measurements from a pitot-static probe positioned along the centerline of the test section. Measurements were collected for speeds ranging from $U_{\infty}=7.6 \mathrm{~ms}^{-1}$ to 
$U_{\infty}=10.4 \mathrm{~ms}^{-1}$, between the inlet and the most aft position of the test panel $(x=1.25 \mathrm{~m})$. After each set of measurements, the threaded rods in the ceiling were adjusted, and the pressure gradient re-checked. This procedure was repeated until zero-pressure-gradient conditions were achieved over the test plate. The spanwise pressure gradient was also checked using the pitot-static probe and a digital level at each of the eight attachment points.

\subsubsection{Test section floor}

The original composite floor was prone to warping due to asymmetric bonding of the aluminum face sheet with the MDF board; an issue, which did not become apparent until approximately two years after construction. To resolve this, the floor was replaced with a continuous, $12.7 \mathrm{~mm}$-thick aluminum plate, which was extended to $1.83 \mathrm{~m}$ from the original length of $1.52 \mathrm{~m}$ to cover the gap at the downstream end of the floor. This also allowed the test panel to be moved further aft for greater boundary layer development downstream of the trip. To minimize the amount of air leakage into the test section, the junctions between the side walls and the aluminum floor were sealed with rubber strips. Remaining gaps at the inlet were sealed using clay caulking, and the entire perimeter of the inlet was sealed using flexible weatherstrips. The test section, in its modified form, is shown schematically in Fig. 22.6, and an image of the interior of the test section is shown in Fig. 2.7.

\subsubsection{Test panels}

A portion of the test section floor was removed, via waterjet cutting, to allow a test panel to be recessed within the floor. The panel was supported by two L-channel sections 


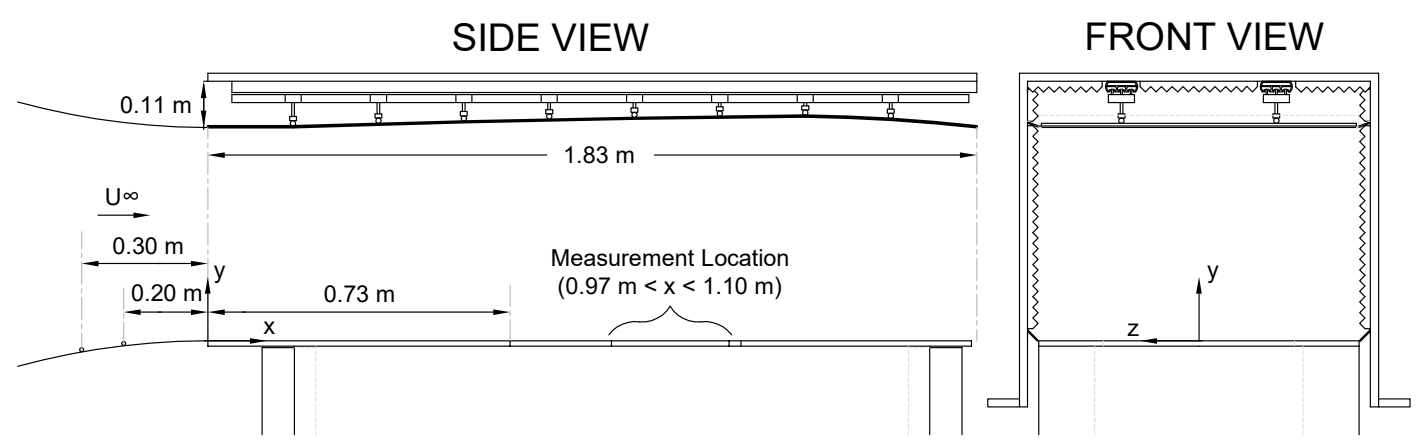

Figure 2.6: Schematic of the test section in its final configuration.

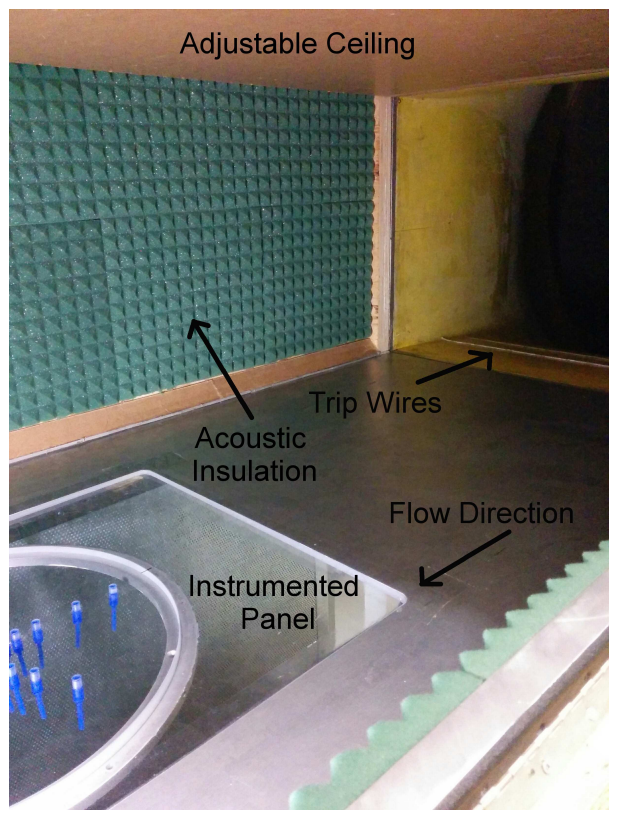

Figure 2.7: Image of the interior of the test section.

fastened to the inner side walls, and the junction between the floor and the panel was sealed with packing tape. Two test panels were machined to position flush-mounted microphones in various spatial arrangements. Both panels were machined out of $19 \mathrm{~mm}$ thick acrylic, to closely approximate a rigid surface, so that fluid-structure coupling can be neglected; a key assumption associated with existing wall-pressure spectrum models. The two microphone arrays are shown schematically in Fig. 2.8, both of which will be described next. 


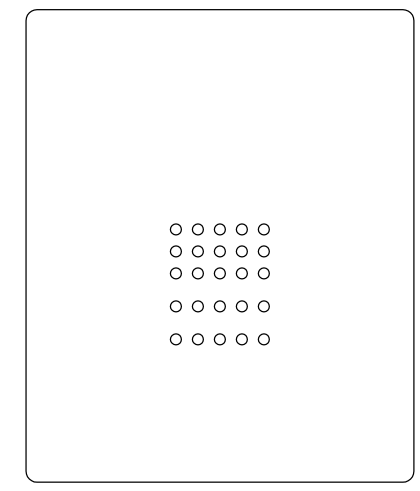

(a)

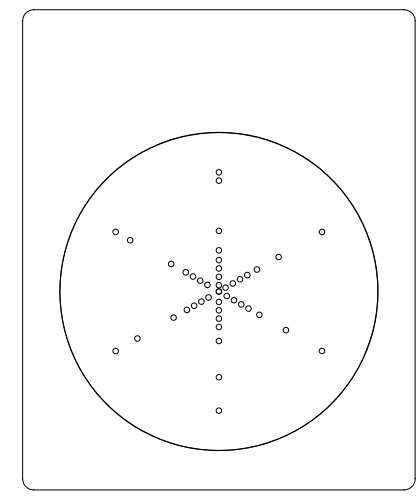

(b)

Figure 2.8: Instrumented test panels for measurement of wall-pressure fluctuations: a) 25-position, rectangular array and, b) 45-position, rotating array.

The first panel was machined with a rectangular array of 25 threaded slots (Fig. 2.8a). Microphones are flush-mounted with the surface of the test panel using specialized, two-piece capsules, which house the microphones (Fig. 2.9a). The upper part of the capsule threads into the panel and forms a collar to stabilize the microphone. The lower piece threads onto the upper piece and supports the bottom of the microphone, allowing precise control over the position of the microphone relative to the surface. Custom, threaded plugs were designed to occupy the vacant holes in the panel. The array permits wall-pressure measurements to be made over a span of $127 \mathrm{~mm}$ and $102 \mathrm{~mm}$ in the streamwise and spanwise directions, respectively. The first row of microphone slots is located $254 \mathrm{~mm}$ from the leading edge of the panel. The first three rows of holes are spaced $25.4 \mathrm{~mm}$ apart, in the spanwise direction, with the last two rows spaced $38.1 \mathrm{~mm}$ apart. The microphone spacing in the spanwise direction is $25.4 \mathrm{~mm}$.

Preliminary measurements by Van Blitterswyk \& Rocha [43, 67] using the first panel, highlighted that much smaller spacing between microphones was required to fully capture the convective nature of the high-frequency wall-pressure signature. The spacing 


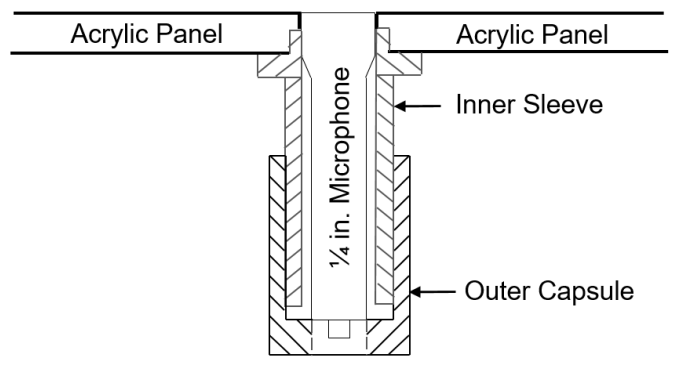

(a)

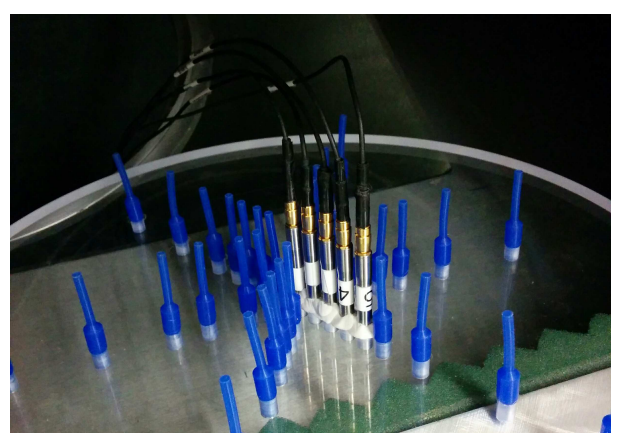

(b)

Figure 2.9: Techniques for flush-mounting microphones: a) custom two-piece threaded capsules for panel 1 and, b) adhesive clay for panel 2.

required for full characterization was estimated by Salze et al. 42 to be $1 \mathrm{~mm}$. This spacing cannot be obtained using standard 1/8-inch or 1/4-inch microphones placed in fixed arrays. A number of researchers have proposed rotating microphone arrays to drastically improve the spatial coverage and resolution of their wall-pressure measurements, using the assumption of statistical stationarity [3, 42, 49]. Stationarity implies that the average statistics of a random signal are independent of time origin, allowing one to characterize the wall-pressure field using separate tests with the microphone array in different orientations. In the work by Arguillat et al. [3] and Salze et al. 42 the microphones were arranged in a linear array, which could be incrementally rotated to capture the convective nature over, a large diameter, with high resolution. Robin et al. [49] proposed multiple linear arrays with radial offsets to achieve a greater spatial coverage, with fewer measurements, and at a spatial resolution on the order of $2 \mathrm{~mm}$. This was considered greatly advantageous for this work due to the limited number of microphones available. Therefore, the microphone arrangement proposed by Robin et al. [49] was used as inspiration for the design of the second test panel.

The circular insert of the second panel (Fig. 2.8b) contains a 45-hole rotating array with six linear patterns, each offset by $60^{\circ}$, and a non-uniform radial distribution along each linear array. The radial offset between adjacent microphone arrays was varied from 
$1.6 \mathrm{~mm}$, between for each of the first four transducers, increasing up to $6.4 \mathrm{~mm}$ at the outer most regions. Without rotation, the panel allows measurements of wall-pressure to be collected with a minimum spacing of $7.1 \mathrm{~mm}$. When rotated by $60^{\circ}$, measurements can be obtained with a spacing of $1.6 \mathrm{~mm}$. To permit such close spacing between microphones, custom capsules could not be used, as with the first test panel. Instead, the microphones were temporarily fixed to the panel with an adhesive clay, and all unoccupied holes were filled with silicon plugs (Fig. 2.9b). To rotate the panel, the circular cut-out is flush-mounted within a rectangular acrylic panel having the same overall geometry as the first panel. To support the cut-out, a large ring bearing was bonded to the underside of the rectangular panel, to which the cut-out was bonded on the inner ring. This enabled the same panel support structure to be used for both panels. The panel-bearing assembly is shown in Fig. 2.10.

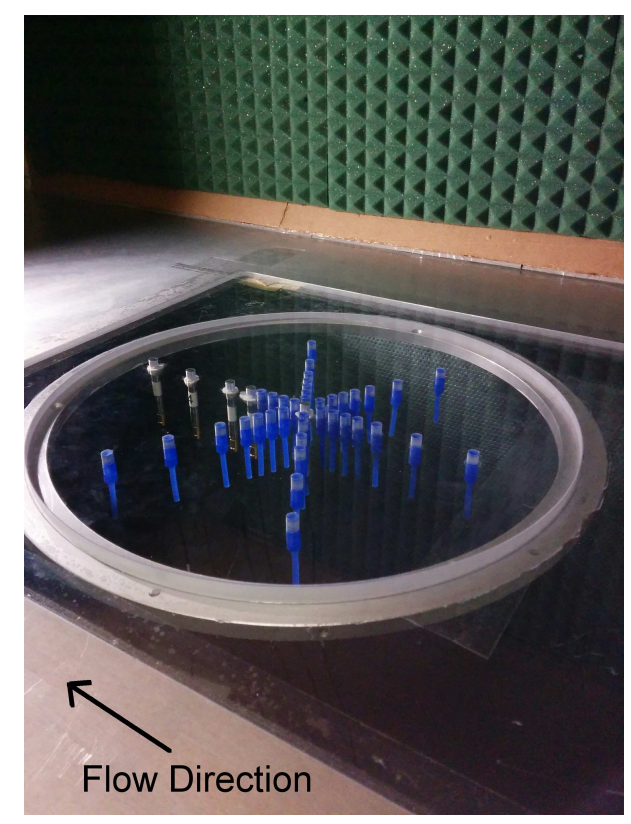

Figure 2.10: Image of the rotating panel assembly installed in the test section floor. 


\subsection{Experimental Techniques}

\subsubsection{Hotwire anemometry system and traverse}

A miniature, platinum-plated, tungsten hotwire anemometer probe, with a single sensor of $5 \mu \mathrm{m}$ in diameter, $d$, and $1.25 \mathrm{~mm}$ in length, $l$, was used to measure boundary layer velocity fluctuations over the test panels. The anemometer system (AN 1003 from AA Lab Systems), operated in a 1:1 bridge configuration and at an overheat ratio of 1.7, was equipped with a built-in amplifier and low-pass filter. A National Instruments (NI) PXIe1071 chassis data acquisition system was used to sample the conditioned signal from the anemometer at $6 \mathrm{kHz}$ for 65 seconds to achieve statistical convergence on mean flow properties. Spectral analysis of signals obtained at $12 \mathrm{kHz}$ verified proper resolution of high-frequency velocity fluctuations. When velocity and wall-pressure measurements were recorded simultaneously, the sampling frequency was increased to $8 \mathrm{kHz}$ to synchronize both instruments.

The probe is suspended by a stem protruding through the slotted ceiling. The stem consists of a hollow tube, from which a tapered sting extends at its end. The sting is used to support a slender, angled steel rod and a small, rapid prototyped, fairing to house the probe support. A schematic of the entire assembly is shown in Fig. 2.11.

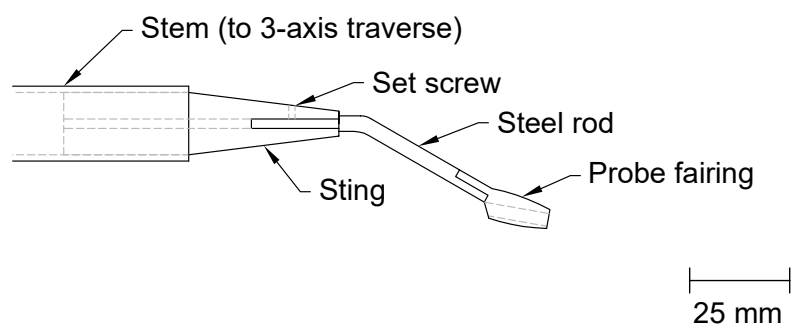

Figure 2.11: Schematic of the hotwire probe support structure. 
The probe then extends from the fairing at an angle of $10^{\circ}$, based on the work by Roberts [85], which was found to minimize probe-induced flow disturbances within the boundary layer. To isolate the hotwire traverse system from the test section, a simple support structure was designed and machined to suspend the traverse system from a rigid gantry structure above the test section (Fig. 2.12a). Mounted on this structure was a three-axis traverse stage, with a travel of $120 \mathrm{~mm}, 120 \mathrm{~mm}$ and $47 \mathrm{~mm}$ in the streamwise, spanwise, and wall-normal directions, respectively. The traverse system is composed of four, Edmund Optics linear stages (Fig. 2.12b) that are connected using custom aluminum brackets. To extend the wall-normal travel, two concatenated precision stages are used, with the first providing $1 \mu \mathrm{m}$ resolution, up to $y=25 \mathrm{~mm}$, and the second stage providing $0.1 \mathrm{~mm}$ resolution between $25 \mathrm{~mm} \leq y \leq 47 \mathrm{~mm}$. The wall-normal stages were mounted onto two perpendicular stages, with $1 \mathrm{~mm}$ resolution, to enable traverses in the streamwise and spanwise directions. The support stem for the probe is finally connected to the first vertical stage via a custom clamp fixture.

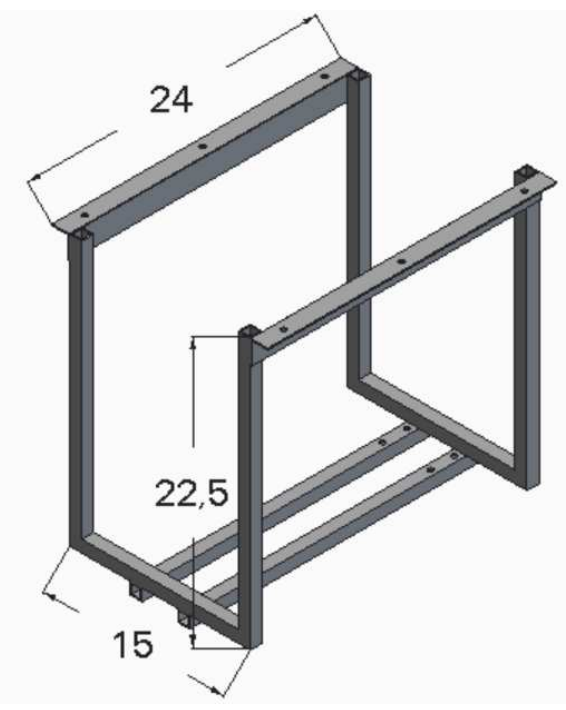

(a)

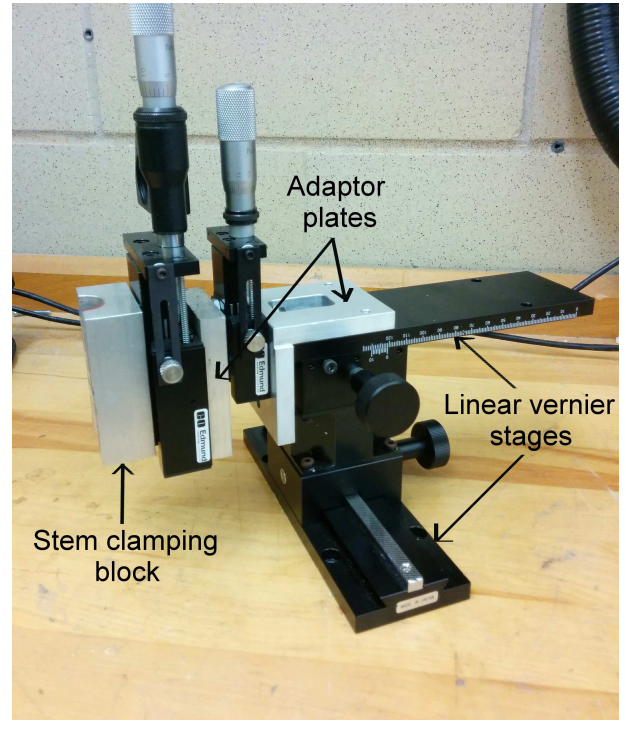

(b)

Figure 2.12: Hotwire traverse system: a) custom frame to suspend three-axis traverse (overall dimensions in inches) and, b) picture of assembled traverse system. 


\subsubsection{Hotwire calibration}

Calibration of the hotwire sensor was performed directly in the wind tunnel. The sensor was positioned in the center of the test section with a pitot-static probe mounted above, and slightly behind, the hotwire sensor so as to not disturb velocity measurements. The hotwire probe was calibrated specifically for the velocity range of interest in the current work $\left(0\right.$ to $\left.12.5 \mathrm{~ms}^{-1}\right)$ at a resolution of $0.9 \mathrm{~ms}^{-1}$. Temperature was measured inside the test section and used to correct voltage measurements using the approach proposed by Bruun [86] (Eq. 2.1)).

$$
E_{c}=E_{a} \cdot\left(\frac{T_{w}-T_{o}}{T_{w}-T_{a}}\right)^{0.5}
$$

In Eq. 2.1, $E_{c}$ and $E_{a}$ denote the hotwire voltage, corrected and uncorrected for temperature effects, respectively, and $T_{w}, T_{o}$ and $T_{a}$ denote the wire temperature, reference temperature, and ambient temperature, respectively. Once corrected, a fourthorder polynomial was used to obtain the calibration coefficients relating velocity to voltage. Typical regression errors were less than $1 \%$, and if larger scatter was observed, the calibration procedure was repeated. A typical calibration curve is shown in Fig. 2.13.

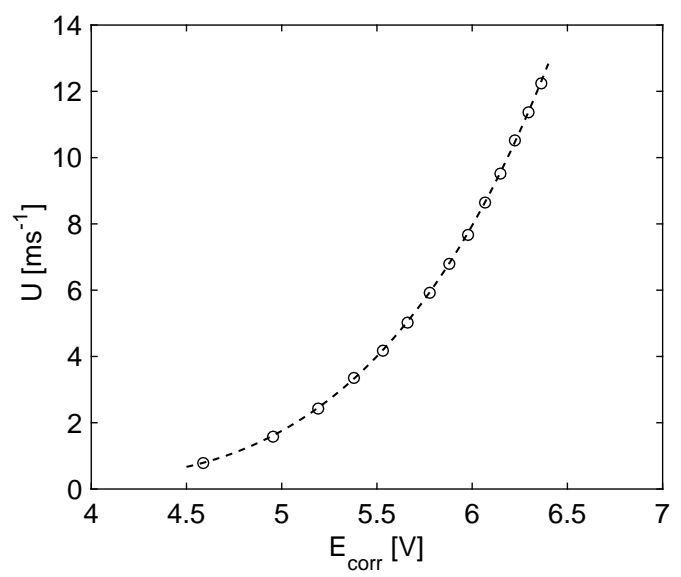

Figure 2.13: Typical hotwire calibration curve. The dashed line denotes a fourth-order calibration polynomial. 
Signal drift in hotwire anemometry measurements is one of the largest sources of error [85, 86] and therefore, the probe calibration was checked frequently; typically after each boundary layer traverse, during the first hour of wind tunnel operation, and every second traverse thereafter. To minimize signal drift, the wind tunnel was run continuously at the target velocity until the temperature reached a quasi-steady value $\left( \pm 0.1^{\circ} \mathrm{C}\right)$. The use of acrylic test panels also minimized the effects of heat transfer near the wall, and therefore no correction for these effects was applied. The measured velocity also has uncertainty associated with the temperature measurement $\left( \pm 0.05^{\circ} \mathrm{C}\right)$, calibration fitting ( $1 \%$ full scale), non-parallel orientation of the probe with respect to the wall $\left( \pm 2.5^{\circ}\right)$, errors in calibration of the freestream velocity with the fan speed $(2 \%$ full scale), and anemometer drift $(280 \mu V)$. The combined uncertainty of the velocity measurements was estimated to be $3 \%$, except for measurements very near the wall, where the error was estimated to be $6 \%$.

\subsubsection{Wall-pressure transducers, calibration and noise filtering}

Up to five, 1/4 inch-diameter, Brüel and Kjaer 4944A type microphones were used to measure wall-pressure fluctuations. The microphones have a flat frequency response, between $50 \mathrm{~Hz}$ and $10 \mathrm{kHz}$, with an upper dynamic frequency limit of $70 \mathrm{kHz}$, and sensitivities ranging between $0.83 \mathrm{mV} / \mathrm{Pa}$ and $0.90 \mathrm{mV} / \mathrm{Pa}$. A single tone calibration, completed for each microphone, gave very similar sensitivities to the values provided by the manufacturer, and therefore, this correction was used for all measurements thereafter. The microphones were outfitted with $0.5 \mathrm{~mm}$-diameter pinhole caps $\left(d^{+}=d u_{\tau} / v=15\right)$, which has been shown to be sufficient for resolving the high-frequency response of the pressure field $\left(d^{+}<19\right.$ and $d^{+}<18$, as proposed by Schewe [37], and Gravante et al. [33], respectively). Standard grid caps were considered in a preliminary study by the author [43], however, it was found that the large effective sensing area associated with these 
caps resulted in unacceptable attenuation of the high frequency response. This will be discussed in greater detail in chapter 4 .

The microphone signals were sampled using the same NI PXIe-1071 data acquisition system at $75 \mathrm{kHz}$ for 65 seconds to achieve convergence of the first two statistical moments of pressure $\left(\langle p\rangle\right.$ and $\left.\sigma_{p}\right)$. The sampling frequency was set based on a Helmholtz resonance of approximately $32 \mathrm{kHz}$ that was measured in a previous study by the author [43]. When correlating wall-pressure and velocity fluctuations, the sampling rate of the microphone signal was reduced to $8 \mathrm{kHz}$.

Regarding measurement uncertainty, the manufacturer's calibration, for each microphone, has an associated uncertainty of $\pm 0.3 \mathrm{~dB}(1.04 \mathrm{~Pa})$, rel. $1 \mathrm{~Pa}$. Incorporated in this value are uncertainties associated with the pre-amplifier gain, non-linearity of the analyzer, repeatability and rounding errors. In addition, the data acquisition card has an input line voltage uncertainty of $\pm 0.1 \%$ of full scale. Other flow-induced sources of error arise, such as attenuation of high-frequency fluctuations, due to spatial averaging of the microphone, and background noise contamination. These sources of uncertainty will be discussed next, however, techniques have yet to be established for characterizing the associated uncertainty. Therefore, a microphone with a calibration constant of $0.83 \mathrm{mV} / \mathrm{Pa}$ has an estimated uncertainty of $4 \%$.

\subsubsection{High-frequency attentuation}

A microphone of finite size is limited in its ability to measure high-frequency pressure fluctuations, as its response is proportional to a spatial average of pressure over its face, causing the microphone to act as a low-pass filter. The level of uncertainty in the high frequency response is difficult to quantify and has received much attention over the past 40 years [10, 37, 62, 63, 87]. Corcos [10], was one of the first to develop a correction for high-frequency attenuation based on the cross-spectral response of the flow, the 
response kernel of a circular transducer, and the Strouhal number. However, White [88] and Lupetow [63] raised concerns that Corcos' correction could lead to over-corrections at high frequencies. Further, Geib Jr. [62] identified that not all transducers exhibit a separable spatial response function in the frequency domain, as was assumed by Corcos [10]. The appropriateness of this correction has still not been definitively confirmed and therefore, this correction was not applied.

\subsubsection{Filtering Background Noise}

Experimental measurements collected in wind tunnels are commonly contaminated by acoustic modes from the facility, which have high spectral energy at low frequencies. Common noise cancellation techniques rely on temporal subtraction of two spanwise transducer signals, separated by a few boundary layer thicknesses, so as to assume the wall-pressure signatures are uncorrelated [89-91]. However, differentiating between background disturbances, and the signal from low-frequency turbulent fluctuations near the edge of the boundary layer, is difficult with this technique. An alternative approach was used by Forest [90], who attempted to eliminate portions of the frequency spectrum based on elevated cross-spectrum amplitudes between two microphones. This approach is more effective for selectively filtering portions of the signal with a low signal-to-noise ratio. Forest [90] used a constant value of 0.08 for the crossspectrum threshold. In this work, cross-spectrum measurements between streamwise velocity fluctuations, at the centerline of the test section, and a reference microphone were used to establish a threshold for filtering out disturbances unrelated to the boundary layer.

The normalized cross-spectrum between the microphone of interest, at location ' $p$ ', and a reference microphone, ' $P$ ', is calculated using Eq. 2.2. For pressure-velocity correlations, the single-sided power spectrum of the reference microphone, $\Phi_{P}(f)$, would 
be replaced by that of the hotwire signal, $\Phi_{u}(f)$, and the cross-spectrum, $\Phi_{p P}(f)$ by $\Phi_{p u}(f)$. The power spectral density and cross-spectral densities were estimated by ensemble averaging over 300 sub records using the formulation from Snarski \& Lupetow [74].

$$
\gamma_{p P}^{2}=\frac{\left|\Phi_{p P}(f)\right|^{2}}{\Phi_{p}(f) \Phi_{P}(f)}
$$

The normalized cross-spectrum distribution, measured between the centerline velocity and wall-pressure fluctuations, is shown in Fig. 2.14 for the flow speeds considered in this work $\left(U_{\infty}=7.6 \mathrm{~ms}^{-1}\right.$ and $\left.U_{\infty}=10.4 \mathrm{~ms}^{-1}\right)$.

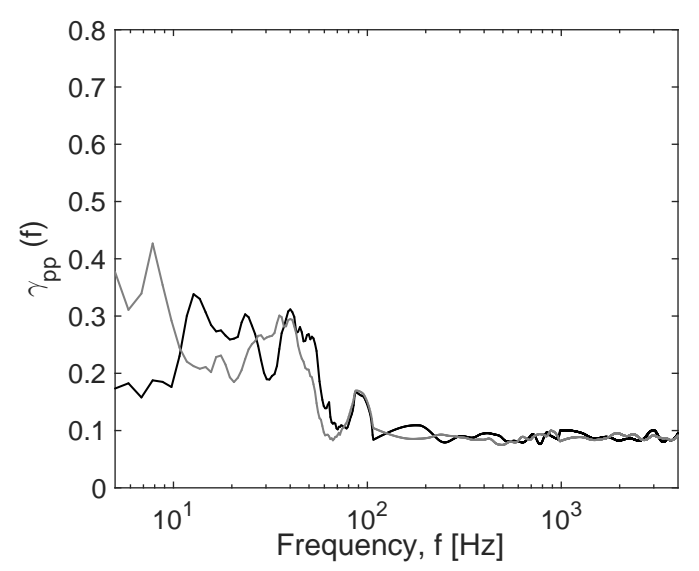

Figure 2.14: Distribution of cross-spectrum energy between freestream velocity fluctuations and wall-pressure fluctuations; 一, $U_{\infty}=7.6 \mathrm{~ms}^{-1} ;-, U_{\infty}=10.4 \mathrm{~ms}^{-1}$.

In all cases, the cross-spectrum is elevated below $55 \mathrm{~Hz}$, indicating that the contributions to the wall-pressure signature are from fluctuations in the freestream or background aberrations. Therefore, the threshold for filtering the complete wallpressure spectrum was then set as the value of the cross-spectrum, between the two microphones, at $55 \mathrm{~Hz}$. 


\subsection{Boundary Layer Trip Device Selection}

With limited distance available for boundary layer development within the test section, a tripping device was required to promote transition. An experimental study was performed to iteratively select the geometry and position of the tripping device, such that a fully-developed boundary layer profile was closely achieved over the over the microphone measurement locations $(0.93 \mathrm{~m} \leq x \leq 1.15 \mathrm{~m})$. Based on techniques found in the literature, the following tripping devices were considered for flow speeds ranging from $7.6 \mathrm{~ms}^{-1}$ up to $10.4 \mathrm{~ms}^{-1}$. To study the relative effectiveness of promoting transition all tripping devices were initiating at $5 \mathrm{~cm}$ downstream from the inlet.

\footnotetext{
${ }^{1}$ Commissioning measurements were used to establish the upper free stream velocity limit for which fully developed conditions were achievable
} 
1. Single Trip Wire: $2.74 \mathrm{~mm}$ diameter.

2. 4 Trip Wires: $2.74 \mathrm{~mm}$ diameter, spaced 5.5 diameters apart ${ }^{2}, 58 \mathrm{~mm}$ streamwise extent.

3. Cylindrical Pins: $10 \mathrm{~mm}$ diameter, $1.5 \mathrm{~mm}$ height, $15 \mathrm{~mm}$ center-to-center spacing, $40 \mathrm{~mm}$ streamwise extent.

4. 3 Rectangular Steps: $3 \mathrm{~mm}$ height, $9 \mathrm{~mm}$ width, $7 \mathrm{~mm}$ spacing, $49 \mathrm{~mm}$ streamwise extent.

5. 3 Velcro Strips: $2 \mathrm{~mm}$ height, $19 \mathrm{~mm}$ width, $57 \mathrm{~mm}$ streamwise extent.

6. 6 Velcro Strips: $2 \mathrm{~mm}$ height, $19 \mathrm{~mm}$ width, $114 \mathrm{~mm}$ streamwise extent 3 .

For $R e_{\theta}<5,000$, the flow field downstream of a trip is influenced by the transition trip mechanism and upstream flow history, such as freestream turbulence levels. Therefore, one cannot decipher if a flow has properly reached a 'fully-developed state' based on Reynolds number alone [56, 61]. As the boundary layer grows, the inner and outer layers (see section 1.2.1) interact strongly creating a strong Reynolds number dependence for the skin friction coefficient, $C_{f}$, boundary layer shape factor, $H$ and wake strength, $\Pi$ [94]. Therefore, each was considered when evaluating the state of the natural and forced-transition boundary layers (Fig. 2.15).

From this study, it was determined that the trip wire and Velcro strips were most effective at promoting fully-developed flow. The trip wire provided comparable stimulation to the Velcro, with the added benefit of a greater development length, and therefore, the trip wire was selected. To maximize the streamwise development length,

\footnotetext{
${ }^{2}$ The trip wire spacing was selected based on the work by Braslow [92], which was shown to reduce the critical Reynolds number for transition.

${ }^{3}$ The streamwise extent of the cylindrical pins, rectangular steps, and Velcro strips, were chosen to closely match that used by Rona \& Soueid [93] under very similar flow conditions.
} 


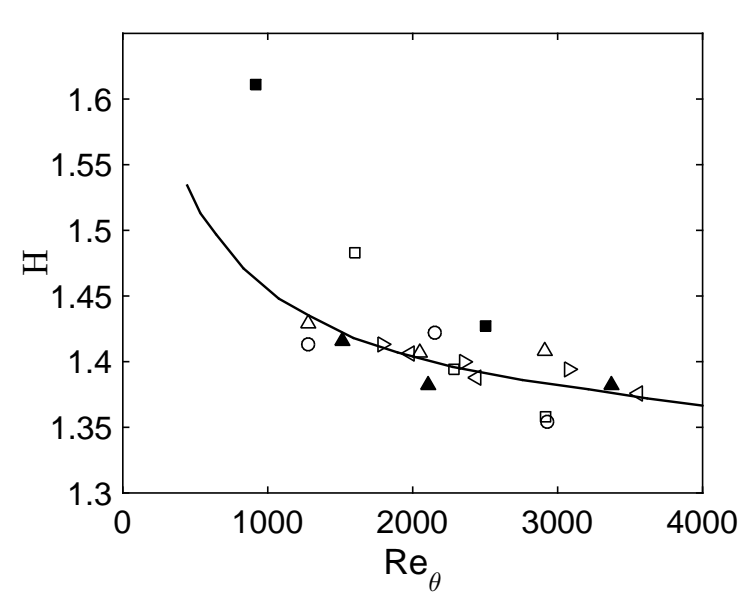

(a)

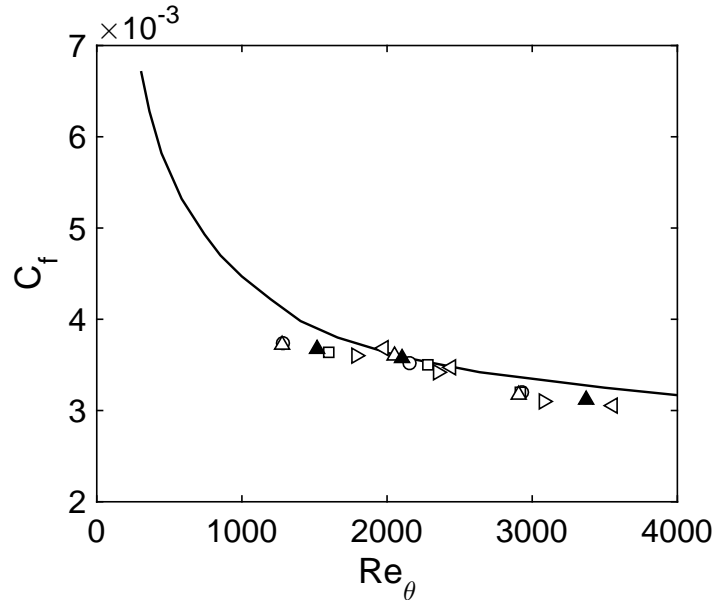

(b)

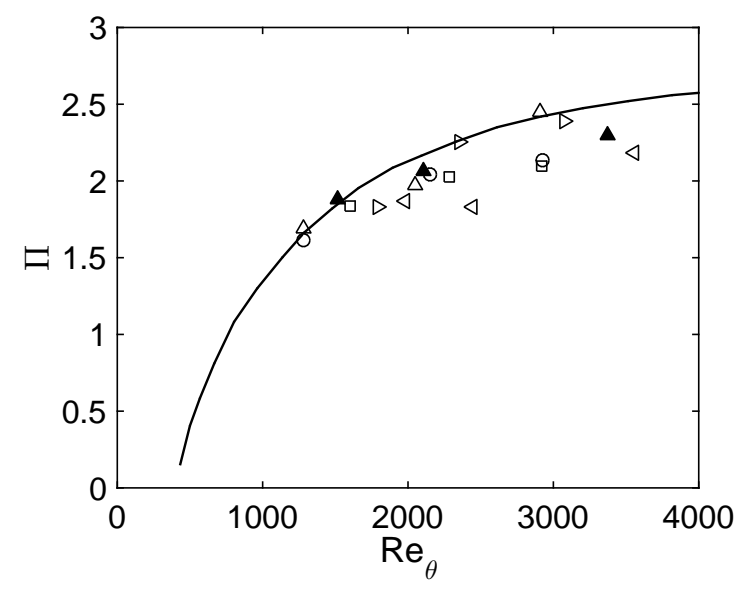

(c)

Figure 2.15: Measured boundary layer parameters compared to ideal, fully-developed boundary layer trends: a) shape factor, b) wake strength, and c) skin friction coefficient. In a) and c) —, empirical trend from Coles [61]; in b) —, empirical trend from Finley [95]; $\mathbf{\square}$, natural boundary layer; $\triangleright, 1$ trip wire; $\triangleleft, 4$ trip wires; $\bigcirc$, cylindrical pins; $\square, 3$ rectangular steps; $\triangle$, 3 Velcro strips; $\triangle$, 6 Velcro strips.

and enhance the level of stimulation imparted to the flow, two $3 \mathrm{~mm}$ trip wires were placed inside the inlet at $x=0.3 \mathrm{~m}$ and $x=0.2 \mathrm{~m}$ upstream of the inlet in the contraction, as inspired by Forest [90]. This trip configuration has an estimated $R e_{h} \approx 795$ and $R e_{\theta} \approx 190$, satisfying the conditions for trip geometry selection put forth by White $\left(R e_{h} \approx 900\right)[96]$ and Compton $\left(R e_{\theta} \approx 200\right)[97 \mid$, respectively. 


\section{Chapter 3}

\section{Statistical Modeling of Wall-Pressure}

\section{Fluctuations}

Due to the stochastic nature of the wall-pressure and velocity fluctuations, within and beneath a TBL, statistical tools are developed to describe their spatial and temporal characteristics. In this chapter, a brief overview of relevant statistics theory is presented, prior to introducing empirical models for statistical descriptors used to study wall-pressure fluctuations. The theory is presented in the form of an arbitrary random function, as these descriptors apply to both wall-pressure and velocity measurements.

\subsection{Statistical Descriptors of Stationary Random Func- tions}

For a specific time, $t$, a random function, $X(t)$, is one that cannot be predicted based on past values. The Probability Density Function (PDF), (Eq. 3.1) $F_{X}\left(x_{n}, t_{n}\right)$, is the set of all possible values that the random variable, $x$, can assume and their associated 
probabilities, $P(X=x)$ (or $P(x)$ ).

$$
F_{X}\left(x_{n}, t_{n}\right)=P\left(X\left(t_{n}\right) \leq x_{n}\right)
$$

The mean, or expected value, of a random function, $\langle X\rangle$, describes the central tendency of $F_{X}\left(x_{n}, t_{n}\right)$ and is defined by Eq. 3.2.

$$
\langle X\rangle=\frac{1}{N} \sum_{n=1}^{N} x_{n}=\sum_{n=1}^{N} x_{n} P(x)
$$

where; $N$, denotes the total number of points in the measured time series and the brackets $\langle\ldots\rangle$ denote an ensemble average. This is also referred to as the first statistical moment of the random variable. The second statistical moment is variance, $\sigma$, as defined in Eq. 3.3 . The square-root of the variance is commonly referred to as the root mean-square value, $X_{r m s}$.

$$
\sigma_{X}=X_{r m s}^{2}=\frac{1}{N} \sum_{n=1}^{N}\left(x_{n}-\langle X\rangle\right)^{2}=\sum_{n=1}^{N}\left(x_{n}-\langle X\rangle\right)^{2} P(x)
$$

The third and fourth statistical moments are known as the skewness, $S_{X}$ (Eq. (3.4)), and kurtosis, $K_{X}$, or flatness, (Eq. 3.5) respectively. Skewness describes the level of asymmetry in the probability distribution, and the kurtosis of a random variable, $K_{X}$, describes the relative weight in the tails of the probability distribution [98]. A high kurtosis is an indication of peaks or intermittent behaviour in the signal [37].

$$
\begin{aligned}
& S_{X}=\frac{1}{N \sigma_{X}^{3}} \sum_{n=1}^{N}\left(x_{n}-\langle X\rangle\right)^{3}=\frac{1}{\sigma_{X}^{3}} \sum_{n=1}^{N}\left(x_{n}-\langle X\rangle\right)^{3} P(x) \\
& K_{X}=\frac{1}{N \sigma_{X}^{4}} \sum_{n=1}^{N}\left(x_{n}-\langle X\rangle\right)^{4}=\frac{1}{\sigma_{X}^{4}} \sum_{n=1}^{N}\left(x_{n}-\langle X\rangle\right)^{4} P(x)
\end{aligned}
$$


The above descriptors provide no information regarding length, or time scale, associated with the random variable. A joint probability function (Eq. (3.6) ) may be used to extract such information using two distinct measurements of the same random variable, say $X$ and $Z$, or time shifting the function as the second instance of the random variable $\left(X_{1}, X_{2}\right)$ [99.

$$
F_{X Y}\left(x_{n}, z_{m}, t_{n}, t_{m}\right)=P\left(X\left(t_{n}\right) \leq x_{n}, Z\left(t_{m}\right) \leq z_{m}\right)
$$

This can be used to define the autocorrelation, $R_{X X}(\tau)$, as a function of time delay, $\tau$, as in Eq. 3.7), or the cross-correlation, $R_{X_{1} X_{2}}(\xi, \eta, \tau)$, as a function of spatial separation ( $\xi=$ streamwise direction, $\eta=$ spanwise direction) and time delay, as in Eq. (3.8).

$$
\begin{gathered}
R_{X X}(\tau)=\sum_{n=1}^{N} \sum_{m=1}^{M} x_{n} x_{m} F_{X Y}\left(x_{n}, x_{m}, t_{n}, t_{m}\right)=\langle X(x, z, t) X(x, z, t+\tau)\rangle \\
R_{X_{1} X_{2}}(\xi, \eta, \tau)=\sum_{n=1}^{N} \sum_{m=1}^{M} x_{n} z_{m} F_{X Y}\left(x_{n}, z_{m}, t_{n}, t_{m}\right)=\langle X(x, z, t) X(x+\xi, z+\eta, t+\tau)\rangle
\end{gathered}
$$

The autocorrelation and cross-correlation functions provide information on the time and space scales, of the random process, respectively, and are commonly expressed in normalized form (Eq. (3.9) and Eq. (3.10), respectively).

$$
\begin{gathered}
r_{X X}(\tau)=\frac{\langle X(x, z, t) X(x, z, t+\tau)\rangle}{\sqrt{\left\langle X(x, z, t)^{2}\right\rangle\left\langle X(x, z, t+\tau)^{2}\right\rangle}} \\
r_{X_{1} X_{2}}(\xi, \eta, \tau)=\frac{\langle X(x, z, t) X(x+\xi, z+\eta, t+\tau)\rangle}{\sqrt{\left\langle X(x, z, t)^{2}\right\rangle\left\langle X(x+\xi, z+\eta, t)^{2}\right\rangle}}
\end{gathered}
$$

For two transducers aligned in the streamwise or spanwise direction, the crosscorrelation shows a definitive peak, when plotted against the time delay, between signals. This peak is centered on the time delay where the two pressure signals are most similar. This time delay, and the spatial separation between the two transducers, can then be used to calculate the 'convection velocity' of the pressure field. 
The aforementioned time domain descriptors can be transformed into the frequency domain to extract information regarding the distribution of energy in the random function. The two primary statistical descriptors include the power spectral density (PSD), which describes the energy in the signal measured at a single point, or the crossspectrum, which describes the spatial coherency of the energy. The single-sided PSD, is computed using the Fourier transform of the autocorrelation of a single ensemble of data, or periodogram, as in Eq. (3.11] [99].

$$
\phi_{X}(f)=\frac{2}{N}\left|\sum_{n=1}^{N} R_{X X}(\tau) e^{-i \omega n T}\right|=\frac{2}{N}\left|\sum_{n=1}^{N} p(t) e^{-i \omega n T}\right|
$$

Similarly, the cross-spectrum, $\Gamma(\xi, \eta, f)$, may be calculated by taking the Fourier transform of the cross-correlation $R_{X_{1} X_{2}}$ or by using Eq. 3.12] [100].

$$
\Gamma(\xi, \eta, f)=|\phi(x+\xi, z+\eta, f)|=\phi_{p}(x, z, f) \phi_{p}(x+\xi, z+\eta, f)^{*}
$$

In Eq. (3.12 '*' denotes complex conjugation. The cross-spectrum is commonly normalized $(\gamma(\xi, \eta, f))$ by the respective single-point power spectra (Eq. (3.13)).

$$
\gamma(\xi, \eta, f)=\frac{|\phi(x+\xi, z+\eta, f)|}{|\phi(x, z, f)|^{0.5}|\phi(x+\xi, z+\eta, f)|^{0.5}}
$$

Consistent estimators for the true PSD and cross-spectrum, with significantly reduced variance, is obtained by averaging several independent periodograms of the random variable. This reduced variance is obtained at the expense of decreased spectral resolution. This process will be described for the PSD, although it also applies to the crossspectrum as well. Spectral leakage is a consequence of computing a periodogram based on a finite time series, which results in artificial energy contributions to the PSD at high frequencies [101]. To mitigate the effects of spectral leakage, by reducing the amplitude 
of discontinuities at the ends of the time series, the time ensembles are multiplied by a window function $W(t)$, creating a windowed ensemble, $X_{w}(t)=X(t) W(t)$. To minimize the effect of the window function, on the resulting amplitudes in the periodogram, the spectrum of the window function should closely resemble that of an impulse function [101]. In this work, the periodograms were windowed using the Hamming window, and 200 ensembles of data, each containing 67,500 points. The window function and ensemble length were selected based on convergence of the first four statistical moments of pressure, and a good compromise between variance and frequency resolution in the spectrum. For the cross-spectrum functions, a greater number of ensembles were required to reduce the variance to an acceptable level. In that case, 400 rectangular windows were used, each containing 67,500 points. The Hamming and rectangular window functions have the form shown in Eq. 3.14, and Eq. 3.15) respectively.

$$
\begin{gathered}
W(t)= \begin{cases}0.5+0.5 \cos \left(\frac{2 \pi t}{T}\right), & |t|<\frac{T-1}{2} \\
0, & \text { otherwise }\end{cases} \\
W(t)= \begin{cases}1, & |t|<\frac{T-1}{2} \\
0, & \text { otherwise }\end{cases}
\end{gathered}
$$

The window-average estimates for the PSD, and cross-spectrum function, are computed using Eq. 3.16) and Eq. 3.17, respectively.

$$
\begin{gathered}
\phi_{w}(f)=\frac{2}{U M L} \sum_{l=1}^{L}\left|\sum_{n=1}^{M} X_{w}(t) e^{-i \omega n T}\right| \\
\gamma_{w}(f)=\frac{1}{L} \sum_{l=1}^{L} \gamma_{l}
\end{gathered}
$$

In Eq. (3.17), $L$ denotes the number of ensemble averages, and $U$ denotes the energy in 
the window function. The windowed periodograms are divided by the energy in the window function to avoid creating bias in the PSD amplitude. The energy in a rectangular window is unity, and was therefore omitted from Eq. (3.17).

As a final note, the autocorrelation, cross-correlation, and their Fourier counterparts assume that the variables of interest (be it pressure or velocity) are statistically stationary and ergodic, or homogeneous. A random variable is said to be statistically stationary if all statistics are invariant on temporal origin [23, 99]. This is analogous to the assumption of steady-state forced vibration. A random function is considered ergodic when the statistics are invariant on spatial origin, and variant only on spatial separation. For wallpressure measurements, the condition of stationarity is reasonably satisfied so long as the boundary layer is quasi-two dimensional, and has reached an equilibrium state. To be considered ergodic, the boundary layer properties must not vary significantly to limit the variance in statistical characteristics over the spatial separations considered. Both of these conditions were verified prior to taking wall-pressure measurements, as will be discussed in section 4.2 ,

\subsection{Empirical Models for Studying Turbulence-Induced Wall-Pressure Fluctuations}

This section presents the empirical models studied in this work for predicting the singlepoint PSD, cross-correlation and cross-spectrum functions, and the convection velocity of wall-pressure fluctuations. 


\subsubsection{Single-point wall-pressure spectum models}

In the current study, the applicability of various existing empirical models are investigated for predicting the PSD at low Mach number flows. The experimental wall-pressure spectra were compared to six empirical models including those developed by: Low-

son [102], Robertson [54], Laganelli [55], Efimtsov [53], Smol'yakov [47], Goody [31], and Rackl \& Weston [30]. The survey of models discussed in this section is not meant to serve as an exhaustive list, and instead as a review of the most popular models developed over the past 50 years.

\subsubsection{Lowson's model}

Lowson [102] developed a model for subsonic and supersonic flow speeds using the empirical curves from Bies [103], which had the form of Eq. 3.18],

$$
\phi(f)=\frac{q^{2}\left(\frac{\overline{p^{2}}}{q^{2}}\right)}{\omega_{o}\left(1+\left(\frac{2 \pi f}{\omega_{o}}\right)^{2}\right)^{3 / 2}}
$$

where:

$$
\omega_{o}=\frac{8 U_{\infty}}{\delta}, \quad \frac{\overline{p^{2}}}{q^{2}}=\frac{0.006^{2}}{\left(1+0.14 M^{2}\right)^{2}} .
$$

In Eq. (3.18), $q$ denotes dynamic pressure, $M$, Mach number, $\delta$, the boundary layer thickness, and $U_{\infty}$, the mean freestream velocity. 


\subsubsection{Robertson's model}

Using further subsonic and supersonic wind tunnel measurements, Robertson [54] adjusted the model proposed by Lowson [102], to improve the predicted high-frequency decay, and increase amplitudes at low frequencies. The dimensional form of the power spectrum is given in Eq. (3.19),

$$
\phi(f)=\frac{q^{2}\left(\overline{\frac{p^{2}}{q^{2}}}\right)}{\omega_{o}\left(1+\left(\frac{2 \pi f}{\omega_{o}}\right)^{0.9}\right)^{2}},
$$

where:

$$
\omega_{o}=\frac{U_{\infty}}{2 \delta}
$$

\subsubsection{Lagaelli's model}

Laganelli [55] made further, more significant, adjustments to Lowson's model [102] to account for viscous effects, compressibility, and heat transfer effects using subsonic and supersonic wind tunnel measurements. Laganelli proposed a model in the form of Eq. 3.20a.

$$
\begin{gathered}
\phi(f)=\frac{q_{\infty}^{2} \delta^{*}\left(0.00002293 F_{c}^{-0.5733}\right.}{U_{\infty}\left[1+F_{c}^{2.867}\left(\frac{2 \pi f \delta^{*}}{U_{\infty}}\right)^{2}\right]} \\
F_{c}=\frac{1}{2}+\frac{h_{w}}{h_{a w}}\left(\frac{1}{2}+r \frac{\gamma-1}{2} M^{2}\right)+0.22 r \frac{\gamma-1}{2} M^{2}
\end{gathered}
$$

In Eq. 3.20b), $F_{c}$ is a transformation function that accounts for compressibility effects. In this work the ratio of heat transfer coefficients for the actual wall to an adiabatic wall 
$\left(h_{w} / h_{a w}\right)$, was assumed to be unity. Further, $\delta^{*}$ represents the boundary layer displacement thickness, and $r$ is a correction factor (typically 0.896).

\subsubsection{Efimtsov's model}

Efimtsov [53] developed two models using extensive flight testing and wind tunnel measurements over subsonic and supersonic Mach numbers. The more recent model will be considered in this study, which describes the PSD as a function of Reynolds number based on friction velocity, $R e_{\tau}$, and Strouhal number, $S h$ (Eq. (3.21)),

$$
\phi(f)=2 \pi \alpha u_{\tau}^{3} \rho^{2} \delta \frac{\beta}{\left(1+8 \alpha^{3} S h^{2}\right)^{\frac{1}{3}}+\alpha \beta R e_{\tau}\left(\frac{S h}{R e_{\tau}}\right)^{\frac{10}{3}}}
$$

where:

$\alpha=0.01, \quad \beta=\left(1+\left(\frac{R e_{\tau_{0}}}{R e_{\tau}}\right)^{3}\right)^{\frac{1}{3}}, \quad S h=\frac{2 \pi f \delta}{u_{\tau}}, \quad u_{\tau}=U_{\infty} \sqrt{\frac{C_{f}}{2}}, \quad R e_{\tau}=\frac{\delta u_{\tau}}{v_{w}}, \quad R e_{\tau_{0}}=\frac{\delta u_{\tau}}{v}$.

In Eq. (3.21), $C_{f}$ denotes the skin friction coefficient, $u_{\tau}$, the friction velocity, $v$, the kinematic viscosity of the freestream, and $v_{w}$ the kinematic viscosity at the wall.

\subsubsection{Smol'yakov's model}

Using several sets of data from the literature, Smol'yakov 47] developed a three-part model to capture the distinct behaviours of the power spectrum in the low-frequency, overlap, and high-frequency ranges. The first term in the following equations describes the fundamental behaviour in each range, with the second term $(\{\ldots\})$ describing the transition between frequency ranges. The low-frequency, overlap, and high-frequency portions of the single-point power spectrum can be predicted using Eq. 3.22a, 
Eq. 3.22b, and Eq. 3.22c, respectively.

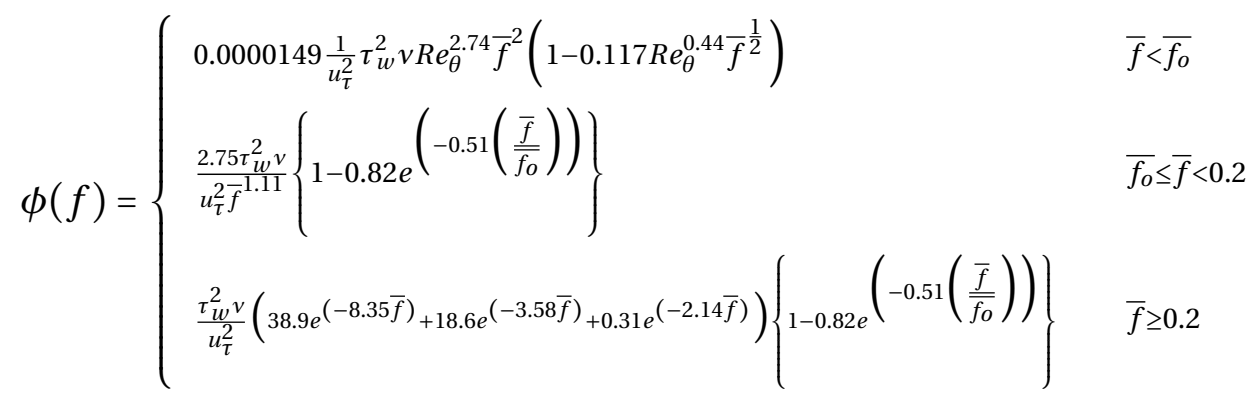

where:

$$
\overline{f_{o}}=49.35 R e_{\theta}^{-0.88}, \bar{f}=\omega v / u_{\tau}^{2}
$$

Further, in Eq. 3.23, $\tau_{w}$ denotes the wall shear stress, and $\operatorname{Re}_{\theta}$, the Reynolds number based on boundary layer momentum thickness.

\subsubsection{Goody's model}

Goody [31] proposed a modified form of the Chase-Howe model [104] to better match experimentally-measured high-frequency decay rates, spectral behaviour in the overlap range and amplify spectral levels at low frequencies. The Goody model has the form of Eq. 3.23,

$$
\phi(f)=\frac{3\left(2 \pi f \tau_{w}\right)^{2}\left(\frac{2 \pi f \delta}{U_{\infty}}\right)^{3}}{\left(\left(\frac{2 \pi f \delta}{U_{\infty}}\right)^{\frac{3}{4}}+0.5\right)^{3.7}+\left(1.1 R_{\tau}^{-0.57}\left(\frac{2 \pi f \delta}{U_{\infty}}\right)\right)^{7}},
$$

where:

$$
R_{\tau}=\left(\frac{u_{\tau} \delta}{v}\right) \sqrt{\frac{C_{f}}{2}}, \quad \tau_{w}=q C_{f}
$$




\subsubsection{Rackl \& Weston's model}

Based on measurements of the wall-pressure fluctuations on the side of a TU-144LL supersonic aircraft, Rackl \& Weston [30] developed correction functions for Efimtsov's model (Eq. (3.21) [53] to adjust the high-frequency roll-off response, and capture a narrow band peak in the spectrum around a Strouhal number of 0.6. The resulting model has the form of Eq. (3.24a), with the correction factors in the form of Eq. (3.24b) and Eq. $3.24 \mathrm{c}$.

$$
\begin{gathered}
\phi(f)=\phi_{\text {Efimtsov }}(f)+\phi_{1}+\phi_{2} \\
\phi_{1}(f)=\frac{1}{4}\left[\tanh \left(\log _{10}\left(\frac{f}{1000}\right)\right)+1\right](M-1.65) \log _{10}(f), \\
\phi_{2}(f)=1.5 e^{\left(-\left(\ln \left(\frac{2 \pi \delta^{*} f}{U_{\infty}}\right)-\ln (0.6)\right)^{2}\right)} .
\end{gathered}
$$

\subsubsection{Coherent energy of wall-pressure fluctuations}

Expanding the measurements at a single point to an array, in the directions parallel and transverse to the flow, allows the characterization of the spatial extent of the energy of wall-pressure fluctuations. Signals measured from each microphone in the array can then be correlated in the time-domain, to establish a 'correlation length scale', or in the frequency domain, to establish a 'coherence length scale', for the wall-pressure fluctuations.

\subsubsection{Space-time cross-correlations and convection velocity}

The conventional approach to extracting the correlation length, $L_{x}$, is to fit an exponential function (Eq. (3.25) to the peaks of the cross-correlation taken from a reference 
microphone to other microphones in the array [7, 9, 11].

$$
\hat{r}_{p p}(\xi, \eta, \tau)=e^{-\frac{|\xi|}{L x}}
$$

Panton et al. [50] proposed that the summation of two exponential functions was more appropriate for capturing the decay of the convected wall-pressure energy. The model proposed by Panton et al. [50] has the form of Eq. (3.26). The two exponential functions suggest that the pressure fluctuations primarily consists of two decay lengths, and the coefficients represent the relative contribution to the total wall-pressure energy at the reference point.

$$
\hat{r}_{p p}(\xi, \eta, \tau)=A e^{-\frac{|\xi|}{L_{1}}}+B e^{-\frac{|\xi|}{L_{2}}}
$$

As previously mentioned, the time delay at peak correlation and spatial separation, between the two transducers, can then be used to calculate the 'convection velocity' of the pressure field. The accuracy of models for the frequency-dependent convection velocity, as proposed by Smol'yakov [47], and Leclerq \& Bohineust [40], are assessed in this work. According to Smol'yakov [105], the convection velocity should vary, as a function of frequency, according to the empirical relation in Eq. (3.27).

$$
\frac{U_{c}}{U_{\infty}}=a \frac{\frac{\omega \delta^{*}}{U_{\infty}}}{1+b\left(\frac{\omega \delta^{*}}{U_{\infty}}\right)^{2}}+c
$$

Smol'yakov reported empirical constants $a, b$ and $c$, with calibrated values of $1.6,16$ and 0.6, respectively. Leclerq \& Bohineust [40] found that, at all frequencies, the measured convection velocity increased with increasing $\xi$. This spatial dependence is not directly accounted for in Smol'yakov's model [105]. 
Based on their measurements, Leclerq \& Bohineust [40] proposed the following model for the frequency-dependent convection velocity (Eq. (3.28)).

$$
U_{c}= \begin{cases}0.07 U_{\infty} \ln \left(\sqrt{v f^{3} \xi^{2}} / u_{\tau}^{2}\right)+0.78 U_{\infty}, & \omega \delta / u_{\tau}<50 \\ U_{c_{M A X}}, & \omega \delta / u_{\tau}=50 \\ U_{c_{M A X}}-0.7 u_{\tau} \ln \left(2 \pi f \delta / 50 u_{\tau}\right), & \omega \delta / u_{\tau}>50\end{cases}
$$

Leclerq \& Bohineust [40] assumed that the peak convection velocity occurs at the same frequency $\left(\omega \delta / u_{\tau}=50\right)$, even though their measurements show a slight spatial dependence. Above this frequency, the convection velocity decreases as a function of the dimensionless number $\sqrt{v f^{3} \xi^{2}} / u_{\tau}$. This dimensionless number was also found to collapse measured cross-spectra, which will be discussed in the following section.

\subsubsection{Cross-spectral density and coherence lengths}

Corcos [10] proposed the most well-known model for predicting spatial coherence between two points. His model describes the spatial coherence by the product of coherent decay in the streamwise and spanwise directions, with the decay constants, $L_{x}$ and $L_{z}$, representing coherence length scales in the respective directions (Eq. (3.29)).

$$
\gamma(\xi, \eta, f) \propto e^{-|\xi| / L_{x}} e^{|\eta| / L_{z}} e^{i \omega \xi / U_{c}}
$$

The length scales can be computed by integrating the cross-spectral density in space, as shown in Eq. 3.30, but is more commonly computed by fitting an exponential function to the cross-spectral density, in the form of Eq. 3.31.

$$
L_{x}=\int_{0}^{\infty} \frac{\gamma(\xi, 0, f)}{\phi(0,0, f)} d \xi, L_{z}=\int_{0}^{\infty} \frac{\gamma(0, \eta, f)}{\phi(0,0, f)} d \eta
$$




$$
L_{x}=e^{-2 \pi f \alpha_{x}|\xi| / U_{c}}, L_{z}=e^{-2 \pi f \alpha_{z}|\eta| / U_{c}} .
$$

The constants, $\alpha_{i}$, are calibrated empirically, and the convection velocity, $U_{c}$, can be determined using temporal correlations, as described in section 3.2.2.1, or for simplicity, it can be assumed to be a constant fraction of the mean freestream speed (typically 0.6$\left.0.8 U_{\infty}\right)$. The inverse dependence of length scale on frequency results in over-prediction of the cross-spectrum at low frequencies. This was addressed by Efimtsov [52], who offered a corrected model of the form,

$$
L_{x}=\delta\left[\left(\frac{2 \pi f \delta a_{1}}{U_{c}}\right)^{2}+\frac{a_{2}^{2}}{\left(2 \pi f \delta / u_{\tau}\right)^{2}+\left(a_{2} / a_{3}\right)^{2}}\right]^{-1 / 2}
$$

where: the empirical constant $a_{1}$ constrains the amplitude of mid- to high-frequency coherence lengths, similar to the constants in Corcos' model [10], the constant $a_{2}$ controls the low-frequency break-away from Corcos' model, and $a_{3}$ controls the lowfrequency roll-off.

Recent experimental measurements by Leclerq \& Bohineust [40], and simulations by Singer [106], have shown that the separation of variables approach, assumed by Corcos [10], for the spatial coherence of wall-pressure fluctuations (Eq. 3.29), underestimates the coherence for off-axis positions. Instead, they reported an elliptic distribution of coherent energy. Leclerq \& Bohineust [40] proposed a model for the normalized streamwise cross-spectrum in the form of Eq. 3.33.

$$
\gamma(\xi, 0, f)=e^{-\sqrt{v f^{3} \alpha_{L}^{2} \xi^{2}} / u_{\tau}^{2}} e^{-\sqrt{\sqrt{\beta_{L}^{2} \xi^{2}}} u_{\tau} / f \delta^{2}}
$$

Reported values for the empirical constants, $\alpha_{L}$ and $\beta_{L}$, are 0.43 and 0.25 , respectively [40]. The same form was assumed for the spanwise cross-spectrum, and the coefficients were found to be $\alpha_{L}=2.98$ and $\beta_{L}=5.53$. To the author's knowledge, the cross-spectrum 
functions have yet to be validated by other experimental measurements. The accuracy of this model will be assessed in the streamwise direction using experimental measurements in this thesis.

\subsection{Event Detection Using Wavelet Transform and Wavelet Cross-Correlation}

An alternative approach to studying the wall-pressure signatures, generated by turbulent motions, is through the wavelet transform. The wavelet transform describes the distribution of energy in the signal as a function of scale and time. The scale information is contained within the wavelet coefficients, which are computed using Eq. (3.34) [12].

$$
w(s, t)=\frac{1}{\sqrt{s}} \int_{-\infty}^{\infty} p_{1}(t) \Psi\left(\frac{t-\tau}{s}\right) d \tau
$$

In Eq. (3.34), $\Psi(t)$, denotes the mother wavelet, $\tau$, denotes the time delay, and, $s$, denotes scale. The mother wavelet is dilated and translated to obtain the energy in the signal, as a function of scale, or frequency. The complex-valued Morlet wavelet, as in Eq. 3.35, was chosen as it provides good space and frequency localization [17], and has been shown to match the form of observed long-life phenomena in turbulence-induced wall-pressure fluctuations [18].

$$
\Psi_{M}(t)=\frac{1}{\sqrt{f_{b} \pi}} e^{i 2 \pi f_{c} t} e^{-t^{2} / f_{b}}
$$

The Morlet wavelet is a plane wave, constrained by a Gaussian envelope of unit width, and is characterized by the center frequency, $f_{c}$, and the envelope bandwidth, $f_{b}$. For the 
purposes of demonstrating the feasibility of this analysis, the default MATLAB smoothing was selected, with a moving average of 7 and 20 points, in scale and time, respectively. The range of wavelet scales were selected to cover frequencies between $55 \mathrm{~Hz}$ and 2,000 Hz. The lower and upper frequency limits were set based on background facility noise, and the upper extent of wall-pressure energy in the PSD, respectively.

Analogous to the space-time cross-correlation, a wavelet cross-correlation function describes the correlations of energy as a function of scale and time delay. Two pressure signals, $p_{1}(t)$ and $p_{2}(t)$, are transformed into the time, $t$, and scale, $s$, components using the wavelet transform (Eq. [3.34), with the corresponding wavelet coefficients defined by $w_{1}(s, t)$ and $w_{2}(s, t)$, respectively. The energy, as a function of scale, in the microphone of interest $\left(w_{2}(s, t)\right)$ is then correlated with the energy in the reference microphone $\left(w_{1}(s, t)\right)$, over a range of time delays, as in Eq. 3.36 [16].

$$
w_{12}(s, \tau)=\lim _{T \rightarrow \infty} \frac{1}{T} \int_{-2 / T}^{2 / T} w_{1}^{*}(t, s) w_{2}(t+\tau, s) d \tau
$$




\section{Chapter 4}

\section{Results \& Discussion}

This section presents the results from several analyses of wall-pressure measurements for the purpose of studying the energy at single-point, and its spatial coherence. Preliminary measurements by Van Blitterswyk \& Rocha [43], with the test section in its initial configuration, are presented in section 4.1. Measurements of the mean flow pressure gradient and boundary layer characterization, following modifications to the wind tunnel, are presented in section 4.2 . The spectral features, characteristics, and scaling parameters for the single point wall-pressure spectrum are then discussed in section 4.3. Comparisons are also made with predictions from several empirical models to assess their accuracy. Wall-pressure spatial correlations and cross-spectrum measurements are then presented in section 4.4. Comparisons with empirical models and measurements from the literature are presented for validation of cross-spectrum measurements. A wavelet cross-correlation analysis is used to extract information about coherent turbulent structures from wall-pressure measurements in section 4.5 . The results from a novel statistical analysis, for processing wavelet transformations of wallpressures measurements, are presented and discussed in section 4.6 . 


\subsection{Single Point Wall-Pressure Spectrum: Original Config- uration}

Preliminary measurements, collected with the test section in its initial configuration are presented first (please see section 2.2). These measurements were collected under the direction of a National Sciences and Engineering Research Council (NSERC) Engage project, in partnership with Bombardier Aerospace. The objective of that study was to assess the accuracy of existing empirical models, for the PSD, at freestream flow speeds of $20 \mathrm{~ms}^{-1}, 30 \mathrm{~ms}^{-1}$ and $40 \mathrm{~ms}^{-1}$. The effects of two flush-mounted microphone cap configurations on the measured wall-pressure spectra were also investigated. At the time these measurements were collected, the hotwire anemometry system had not been acquired. Therefore, empirical relations were used to estimate integral boundary layer parameters required in empirical models for the spectrum ( $\delta$ (Eq. (4.1)), $\delta^{*}$ (Eq. (4.2)), $\theta$ (Eq. (4.3)) and $C_{f}$ (Eq. (4.4))).

$$
\begin{gathered}
\delta=0.37 x \operatorname{Re}_{x}^{-1 / 5}\left(1+0.144 M^{2}\right)^{0.35} \\
\delta^{*}=\frac{\delta\left(1.3+0.43 M^{2}\right)}{10.4+0.5 M^{2}\left(1+2 \mathrm{e}-8 R e_{x}\right)^{1 / 3}} \\
\theta=\frac{\delta}{10.4+0.5 M^{2}\left(1+2 \mathrm{e}-8 R e_{x}\right)^{1 / 3}} \\
C_{f}=0.37\left(\log _{10} R e_{x}\right)^{-2.584}
\end{gathered}
$$

In Eq. (4.1) - Eq. (4.4), $R e_{x}$ denotes the Reynolds number based on distance from the boundary layer origin, $x$ (assumed to start at the inlet). Wall-pressure spectra measured under these conditions, using a standard $1 / 4$ inch grid cap and a $0.5 \mathrm{~mm}$-diameter pinhole cap on the microphones, are shown in Fig. $4.1 \mathrm{a}$ and Fig. $4.1 \mathrm{~b}$, respectively. Both sets 
of measurements are truncated just below the resonant frequency for the microphone cap.

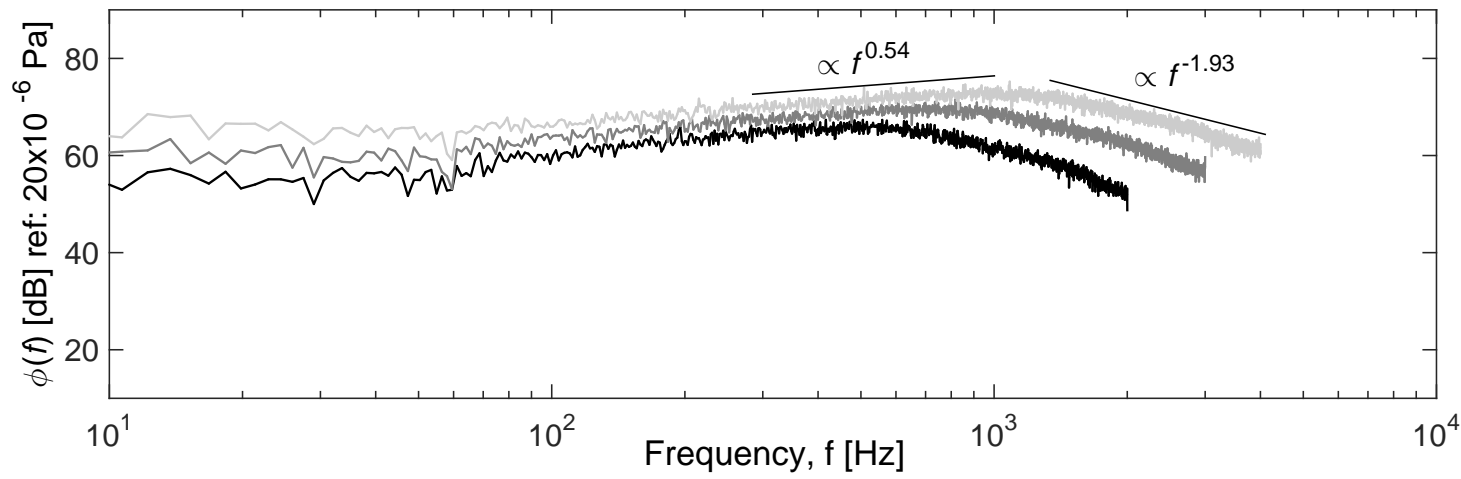

(a)

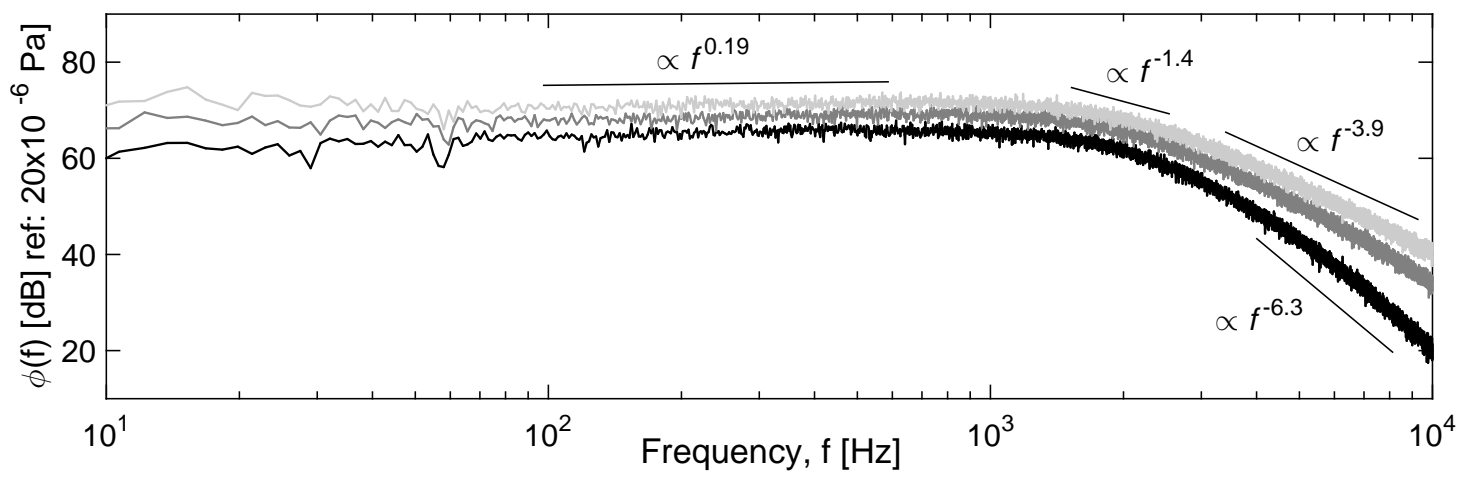

(b)

Figure 4.1: Single-point wall-pressure spectrum at $x=1.10 \mathrm{~m}$ as measured using: a) a grid cap microphone, and b) a $0.5 \mathrm{~mm}$-diameter pinhole microphone; - $\operatorname{Re}_{\theta} \approx$ 2,$516 ;-, R e_{\theta} \approx 3,576 ;-, R e_{\theta} \approx 4,500$. $R e_{\theta}$ estimated based on predicted $\theta$ (Eq. (3.3).

The background noise filtering technique used in this phase of work was adopted from Simpson et al. [89]. Using this approach, it is assumed that the background noise and turbulence-induced noise are separable at any frequency. The noise can then be removed through temporal subtraction of the measured pressure signal at the reference microphone, from that measured at the point of interest, and taking the meansquare value of the result. It is further assumed that the turbulence contributions are not 
correlated so long as the two microphones are separated by at least $\delta$ in the spanwise direction. This technique enabled spectral energy to be captured for frequencies as low as $1 \mathrm{~Hz}$; however, neither spectra exhibited the expected slope, proportional to $f^{2}$, in this range. Over the mid-frequency range, the measured spectra exhibited an increase in energy proportional to $f^{0.19}$, which is in excellent agreement with expected trends $[11,29,33,40,42,43]$. The grid cap measurements show a greater spectral energy slope, proportional to $f^{0.54}$, but one that remains on the order of magnitude of the expected behaviour.

Measurements with the grid cap configuration show a spectral slope proportional to $f^{-1.93}$ beyond $1 \mathrm{kHz}$. This exceeds the commonly reported slope of $f^{-1}$ for the overlap range, which is believed to be a result of spatial attenuation due to the larger effective transducer size. Moreover, the grid cap measurements are truncated at much lower frequencies, compared to the pinhole cap, due to resonance from the slotted surface of the cap. The spectra measured using the pinhole configuration are in better agreement with the literature over the mid- and overlap-frequency ranges, with the decay rate transitioning briefly through $f^{-1.4}$, prior to reaching $f^{-3.9}$, for $f \geq 2,000 \mathrm{~Hz}$.

The estimated $R e_{\theta}$, for the flow conditions tested, indicates that an overlap region should be identifiable at the higher speeds $\left(R e_{\theta} \geq 3,500\right.$ [47]); however, both wall-pressure spectra have minimal frequency bands over which the expected overlap frequency range can be identified. This suggests that the boundary layer, above the measurement location, has not reached a fully-developed state. This was confirmed through spectral normalization, which showed that spectra in this frequency range could not be collapsed using any set of scaling variables [43]. Therefore, the experimental measurements could not be used to properly assess the accuracy of existing empirical models, as all models assume fully-developed TBL conditions. Full details of this study are provided in reference [43]. 


\subsection{Mean Flow Pressure Gradient and Wall Boundary}

\section{Layer}

A hotwire anemometry system was acquired to verify that the boundary layers in the preliminary measurements were not fully-developed, and then establish a proper range of flow speeds under which fully-developed conditions could be achieved. Measurements of the boundary layer velocity profile indicated that the boundary layers, for $U_{\infty}=20-40 \mathrm{~ms}^{-1}$, was indeed not fully-developed. The measured streamwise velocity profile, at the centerline of the test section, also showed a moderate favourablepressure-gradient due to rapid boundary layer growth along the top and side walls of the test section. To correct these issues, several modifications were made to the test section, as detailed in section 2.2 . A tripping device and reduced flow speeds $\left(U_{\infty} \leq 10.4 \mathrm{~ms}^{-1}\right)$ were required to achieve fully-developed TBL conditions over the test panel.

Two flow speeds $\left(U_{\infty}=7.6 \mathrm{~ms}^{-1}\right.$ and $\left.U_{\infty}=10.4 \mathrm{~ms}^{-1}\right)$ were considered for more extensive studies of the wall-pressure fluctuations. After installation of the ceiling, the streamwise pressure gradient was sampled at four speeds, between $7.6 \mathrm{~ms}^{-1}$ and $10.4 \mathrm{~ms}^{-1}$, using a pitot-static probe between the inlet and the most aft position of the test panel, $x=1.20 \mathrm{~m}$, to ensure zero-pressure-gradient conditions were achieved. The evolution of the streamwise and spanwise pressure gradient $\left(C_{p x}=1-U / U_{i n l e t}\right.$, $\left.C_{p z}=1-U / U_{z=0}\right)$ is shown in Fig. $4.2 \mathrm{a}$ and Fig. $4.2 \mathrm{~b}$, respectively. The centerline velocity gradient, over the test panel, was also confirmed using hotwire measurements. The Clauser parameter (Eq. 4.5) [107] was used to verify that the conditions over the panel approximately represent zero-pressure-gradient.

$$
\beta_{C}=\frac{\delta^{*}}{u_{\tau}} \frac{d p}{d x} \approx 0
$$




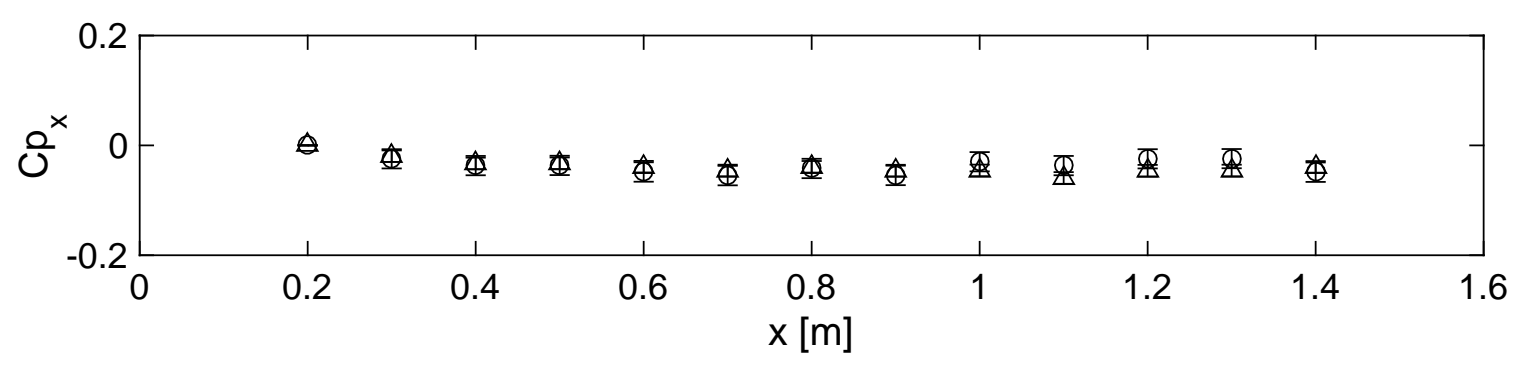

(a)

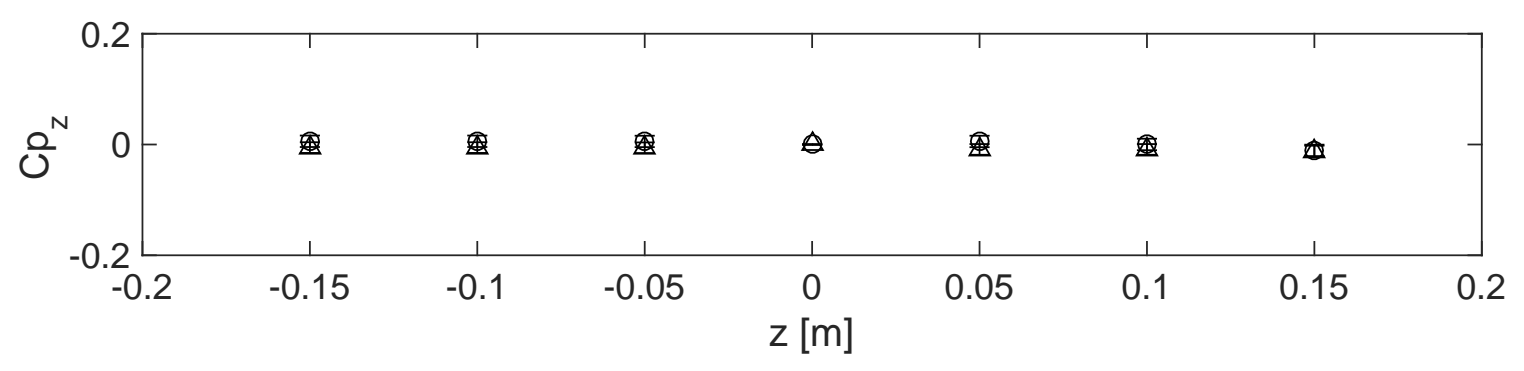

(b)

Figure 4.2: Mean pressure gradient profiles measured within the test section: a) streamwise, and b) spanwise at $x=1.05 \mathrm{~m} ; \bigcirc, U_{\infty}=7.6 \mathrm{~m} / \mathrm{s} ; \triangle, U_{\infty}=10.4 \mathrm{~m} / \mathrm{s}$.

Over the test panel $(0.9 \mathrm{~m} \leq x \leq 1.2 \mathrm{~m}), \beta_{C}$ varied between -0.031 and -0.054 for $U_{\infty}=7.6 \mathrm{~ms}^{-1}$ and $U_{\infty}=10.4 \mathrm{~ms}^{-1}$, respectively. For moderate adverse and favourble pressure gradients, $\beta_{c}$ is expected to have values near 1.0 and -1.0 , respectively [58]. Therefore, for all practical purposes, this represents zero-pressure-gradient conditions. The very small pressure gradient is likely due to separated flow and boundary layer growth along the insulated sidewalls, the effects of which could not be fully removed with the travel of the ceiling supports.

Measured boundary layer profiles of viscous-normalized, mean velocity and normalized Reynolds stress, over the center of the panel $(x=1.05 \mathrm{~m})$, are shown in Fig. 4.3a and Fig. 4.3b, respectively. The integral boundary layer parameters, listed in Table 4.1, were computed from the mean velocity profiles. 


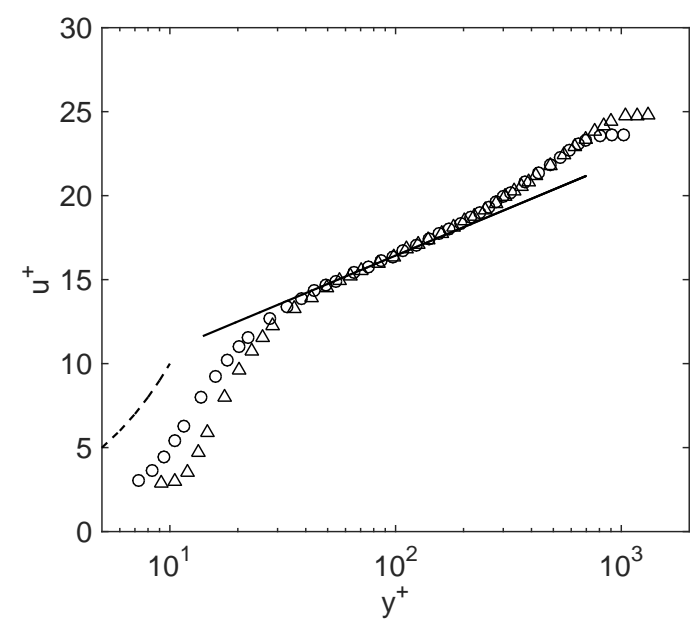

(a)

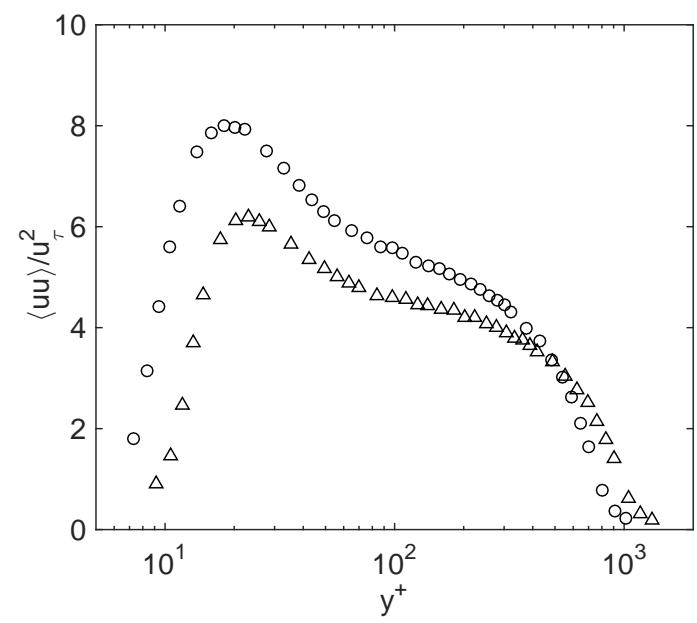

(b)

Figure 4.3: Boundary layer characterization over the center of the test panel $(x=1.05 \mathrm{~m})$ : a) viscous-normalized, mean velocity profiles, and b) normalized Reynolds stress distributions; $\bigcirc, U_{\infty}=7.6 \mathrm{~m} / \mathrm{s} ; \triangle, U_{\infty}=10.4 \mathrm{~m} / \mathrm{s}$. In a): - - -, denotes $u^{+}=y^{+}$, and 一, denotes the logarithmic law $\left(u^{+}=1 / 0.41 \ln \left(y^{+}\right)+5.1\right)$.

\begin{tabular}{llllll}
\hline$U_{\infty}[\mathrm{m} / \mathrm{s}]$ & $U_{\tau}[\mathrm{m} / \mathrm{s}]$ & $\delta[\mathrm{mm}]$ & $\delta^{*}[\mathrm{~mm}]$ & $\theta[\mathrm{mm}]$ & $R e_{\theta}$ \\
\hline 7.6 & 0.34 & 32.6 & 5.1 & 3.5 & 1886 \\
10.4 & 0.44 & 32.5 & 5.0 & 3.5 & 2546 \\
\hline
\end{tabular}

Table 4.1: Measured boundary layer properties at $x=1.05 \mathrm{~m}$

The boundary layer was measured over the test panel at three streamwise locations $(x=0.97 \mathrm{~m}, x=1.05 \mathrm{~m}, x=1.10 \mathrm{~m})$, and three spanwise locations $(z=0 \mathrm{~m}$, $z= \pm 0.06 \mathrm{~m}$ ), at each of the streamwise positions, to ensure the boundary layer had reached a quasi-equilibrium state (i.e.: satisfies the criterion for ergodicity (section 3.1)). This was verified using the criterion defined by Castillo \& Wang [108] (Eq. 4.6).

$$
\Lambda=\frac{\theta}{\rho U_{\infty}^{2} d \theta / d x} \frac{d p_{\infty}}{d x}
$$

For equilibrium boundary layers, $\Lambda$ should be close to zero. In this work, $\Lambda$ was calculated to be -0.01 and -0.003 for $R e_{\theta}=1,886$ and $R e_{\theta}=2,546$, respectively, confirming that 
the boundary layers were ergodic for the purposes of evaluating empirical models for the single-point wall-pressure spectrum.

\subsection{Single-Point Wall-Pressure Spectrum - Final Configu- ration}

The wall-pressure spectra for $R e_{\theta}=1,886$ and $R e_{\theta}=2,546$ are presented in Fig. 4.4. Also shown in Fig. 4.4 is the cross-spectrum distribution between the microphone of interest and the reference microphone, which was used to filter background contamination using the procedure outline in section 2.3.3.2. This noise-filtering approach considers the time-averaged correlation between the two microphones, rather than assuming that the difference in signals, at any instant, can be attributed to background noise. This results in more reliable isolation of background noise from flow-related, low-frequency pressure signatures. The cross-spectrum was computed between the microphone of interest $(z=0 \mathrm{~m})$, and a reference microphone located $0.142 \mathrm{~m}(z \approx 4 \delta)$ away in the spanwise direction.

The single-point spectra show two distinct power-law regions: an overlap range where the energy decays proportional to $f^{-0.76}(200 \mathrm{~Hz}<f<700 \mathrm{~Hz})$, and a highfrequency range $\left(f>1,500 \mathrm{~Hz}\right.$ ), where the energy decay ranges from $f^{-4.9}$ (for $R e_{\theta}=1,886$ ), to $f^{-6.6}$ (for $R e_{\theta}=2,546$ ). These spectrum decay rates are in good agreement with the literature [11, 32, 38, 39]. The more rapid decay, in the high-frequency region of the spectrum, at $R e_{\theta}=2,546$, may be due to the reduced Reynolds stress below the buffer layer $\left(y^{+}<30\right.$ in Fig. 4.3b). At $R e_{\theta}=2,546$, there appears to be a transition region between the overlap and high-frequency ranges, where the spectrum decays proportional to $f^{-3.1}$, which is slightly higher than the expected decay, proportional to 

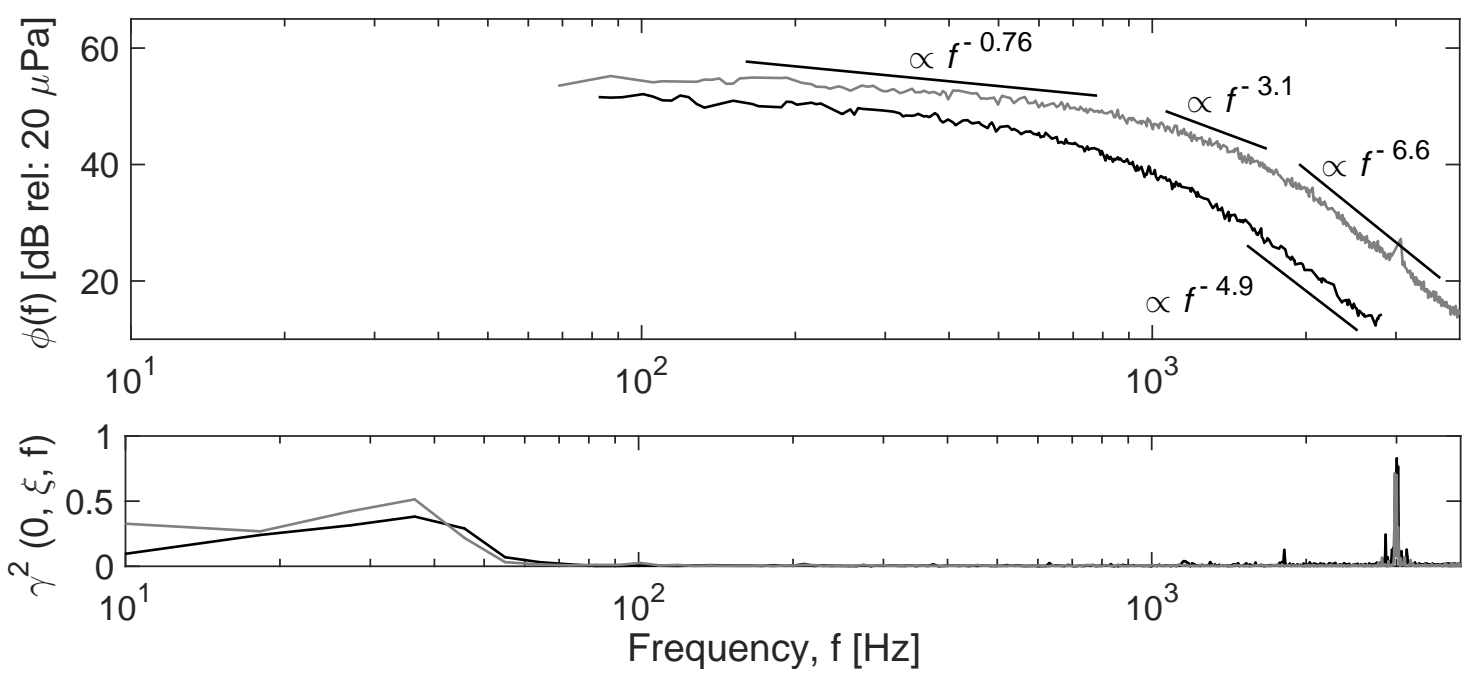

Figure 4.4: Single point wall-pressure spectrum as measured at $x=1.10 \mathrm{~m}$, and crossspectrum with reference microphone used for filtering: $-, R e_{\theta}=1,866 ;-, R e_{\theta}$ $=2,546$.

$f^{-7 / 3}$, as reported in the literature [11, 29, 33, 37]. The spectra exhibit other expected features for low Reynolds number boundary layers, including: a lengthening of the overlap region and a subtle increase in peak frequency, with increasing $R e_{\theta}$.

Spectral normalization is required to compare the current measurements with those taken at higher speeds (section 4.1), and others from the literature. Particular attention is given to the high-frequency and overlap regions of the spectrum, to assess the effects of the test section modifications. There is a general agreement in the literature that the high-frequency range of the spectrum collapses when normalized using inner-layer scales, $\phi(f) u_{\tau}^{2} / \tau_{w}^{2} v$, as a function of $\omega v / u_{\tau}^{2}$ [29, 32, 39, 42]. In Fig. 4.5a, the normalized spectra, measured for $R e_{\theta}=1,886$ and $R e_{\theta}=2,546$, are compared against the normalized spectra measured at $R e_{\theta} \approx 4,500[43]$, and measurements from the literature. The innerlayer normalized spectra are in excellent agreement with other measurements from the literature, and collapse well over the majority of the measured frequency range. This suggests a strong influence of inner-layer turbulence on the wall-pressure fluctuations. The spectra do not collapse as well near the spectrum peak, suggesting that turbulent 
motions contributing energy in this range have larger length and time scales, and likely originate in the outer layer.

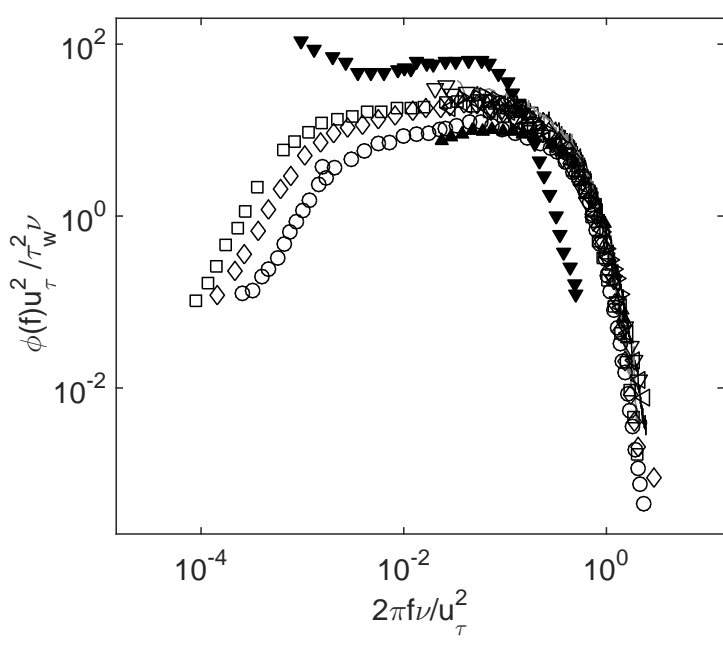

(a)

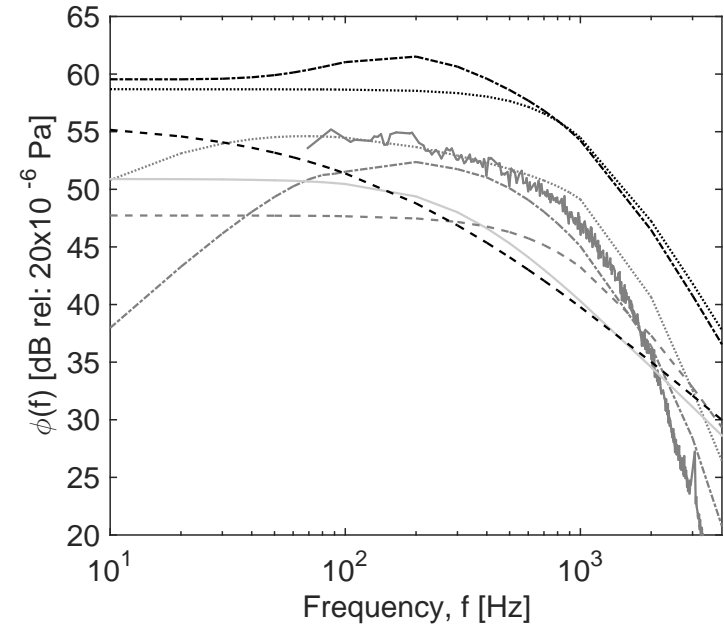

(b)

Figure 4.5: Experimental power spectra of wall-pressure: a) normalized by inner variables and compared to previous measurements by the authors and other literature, and b) compared to the models by Lowson [102], Robertson [54], Laganelli [55], Efimtsov [53], Smol'yakov [47], Goody [31], and Rackl \& Weston [30]; ○, FC 3,$386 ; \diamond$, FC 4,487; $\square$, FC 6,025; $\nabla$, VBR 2,517; $\Delta$, SC 1,400; $\triangle$, GR 1,577; $\triangleright$, GR 2,$953 ; \triangleleft$, GR 3,509; $\nabla$, GR 6,241; —, $R e_{\theta}=1,886 ;-, R e_{\theta}=2,546 ; \ldots .$. , Efimtsov model; - -. , Rackl \& Weston model; ....., Goody model; -. - , Smol'yakov model, - , Laganelli model, ---, Lowson model, ....., Robertson model. The measurements from the literature are identified using a combination of letters, indicating the authors, and numbers, indicating the $R e_{\theta}$ of the data set: $\mathrm{FC}=$ Farabee \& Casarella [29], VBR = Van Blitterswyk \& Rocha [43], SC = Schewe [37], GR = Gravante et al. [33.

The spectrum measured at $R e_{\theta} \approx 4,500$ do not collapse onto these measurements, nor measurements from the literature. The vertical shift in normalized spectrum energy is most likely due to an over-prediction of the wall shear stress derived from predicted boundary layer parameters (section 4.1). Aside from this, it is clear that shape of the wall-pressure spectrum has been altered significantly by the test section modifications, 
showing higher spectrum energy in the overlap frequency range, which brings the normalized spectrum in line with expected levels from the literature for similar $R e_{\theta}$.

The accuracy of existing empirical models is briefly addressed using the new measurements. The wall-pressure spectrum measured for $R e_{\theta}=2,546$ is compared against predictions from the models by Lowson [102], Robertson [54], Laganelli [55], Efimtsov [53], Smol'yakov [47], Goody [31], and Rackl \& Weston [30] as shown in Fig. 4.5b. The agreement between the models in the low-frequency region cannot be addressed, due to background noise contamination (Fig. 4.4), and therefore, discussions are focussed on the overlap and high-frequency regions.

From Fig. 4.5b, it is observed that the models by Efimtsov [53] and Rackl \& Weston [30] are not suitable for modeling the low-Reynolds number wallpressure spectrum. These models over-predict the spectrum amplitude by approximately $5 \mathrm{~dB}$ over the entire frequency range, and under-predict the highfrequency decay. This is expected, as the two-part model is extrapolated to much lower Reynolds numbers than those used to develop these models $\left(33,000 \leq R e_{\theta} \leq 255,000\right)$. The same can be said for the models by Lowson [102], Robertson [54] and Laganelli [55], as these models were developed for transonic and supersonic flow speeds. Extrapolation of these models results in an under-prediction of spectrum amplitudes for $f \leq 2,000 \mathrm{~Hz}$, and the high-frequency spectrum decay rate. These models also do not predict an energy peak in the spectrum [53-55, 102], while others do [30, 31, 47]. As expected, the models by Smol'yakov [47] and Goody [31], developed for low Reynolds numbers, are in better agreement with the measurements.

The accuracy of the empirical models is quantified using a number of metrics, including: peak frequency, $f_{\text {Peak }}$, spectrum amplitude, $A$, and power law growth/decay rates, $D$, at the center of each frequency range, as shown in Table 4.2 . In Table 4.2 , $e$, denotes the error, relative to the measured properties at $R e_{\theta}=2,546$, and the amplitude is 
defined in decibels, relative to $20 \mu \mathrm{Pa}$. Spectral amplitude errors were computed based on pressure, and error in the spectrum slopes are computed based on the power-law exponent, which describes the decay of spectral energy in the overlap and high frequency ranges.

\begin{tabular}{|c|c|c|c|c|c|c|c|c|c|}
\hline $\begin{array}{l}\text { Spectrum/ } \\
\text { Model }\end{array}$ & $\begin{array}{l}f_{\text {Peak }} \\
{[\mathrm{Hz}]}\end{array}$ & $\begin{array}{l}A_{o}^{*, * * *} \\
{[\mathrm{~dB}]}\end{array}$ & $\begin{array}{l}e^{* *} \\
(\%)\end{array}$ & $D_{o}^{* * *}$ & $\begin{array}{l}e \\
(\%)\end{array}$ & $\begin{array}{l}A_{h}^{*, * * *} \\
{[\mathrm{~dB}]}\end{array}$ & $\begin{array}{l}e^{* *} \\
(\%)\end{array}$ & $D_{h}^{* * *}$ & $\begin{array}{l}e \\
(\%)\end{array}$ \\
\hline$R e_{\theta}=2,546$ & 87 & 52.2 & - & $\omega^{-0.76}$ & - & 28.8 & - & $\omega^{-6.6}$ & - \\
\hline Lowson 102] & N/A & 46.6 & -72.7 & N/A & - & 34.9 & 310.2 & $\omega^{-3.0}$ & -54.5 \\
\hline Robertson [54 & $\mathrm{N} / \mathrm{A}$ & 44.8 & -82.0 & $\mathrm{~N} / \mathrm{A}$ & - & 33.4 & 190.4 & $\omega^{-1.7}$ & -74.4 \\
\hline Laganelli [55] & $\mathrm{N} / \mathrm{A}$ & 46.0 & -76.0 & $\mathrm{~N} / \mathrm{A}$ & - & 32.7 & 147.2 & $\omega^{-2.0}$ & -70.5 \\
\hline Efimtsov [53] & $\mathrm{N} / \mathrm{A}$ & 57.9 & 271.5 & $\mathrm{~N} / \mathrm{A}$ & - & 44.3 & 3,473 & $\omega^{-3.1}$ & -53.0 \\
\hline Smol'yakov [47] & 100 & 50.6 & -30.8 & $\omega^{-1.1}$ & 44.7 & 40.3 & 1,332 & $\omega^{-4.7}$ & -28.8 \\
\hline Goody [31 & 80 & 8.1 & -3.4 & $\omega^{-0.7}$ & -7.9 & 36.3 & 470.2 & $\omega^{-5.0}$ & -24.2 \\
\hline Rackl \& Weston [30] & 200 & 59.1 & 389.8 & $\omega^{-1.9}$ & 150 & 45.3 & 4,398 & $\omega^{5.2}$ & -20.9 \\
\hline
\end{tabular}

${ }^{*}$ : Amplitudes extracted at $f=450 \mathrm{~Hz}$ and $f 2,500 \mathrm{~Hz}$ for the overlap and high-frequency . ranges, respectively.

**: Error in amplitude calculated based on Pa and not dB.

***: 'o' denotes overlap and ' $h$ ' denotes high-frequency

Table 4.2: Accuracy of empirical models for predicting experimental wall-pressure spectrum

As shown in Table 4.2, the Goody model is slightly more accurate in predicting the overlap spectrum amplitude and decay rate, with an accuracy of $3.4 \%$ and $7.9 \%$, respectively. The Goody model is also most accurate for predicting the location of the spectrum peak and the rate of decay in the overlap region. The Goody model and Smol'yakov model bound the high-frequency spectrum amplitude up to approximately 2,000 $\mathrm{Hz}$, beyond which the measurements decay slightly faster than predicted by either model $\left(f^{-6.6}\right)$. Coincidental crossings of the Lowson, Laganelli, and Robertson models with 
the experimental spectrum, at the center frequency of the high-frequency range, are responsible for the lower error associated with these models. However, none of these models accurately predict the shape of the spectrum, nor do they predict a peak frequency. Therefore, the comparisons of empirical models with experimental spectra suggest that the Goody model [31] is most appropriate for low Reynolds number flows.

For modeling wall-pressure excitation, it is important to not only have a complete description of the spectrum at a single point, but also its evolution in space. This is most commonly described using the 'correlation length', derived from the cross-correlation in the time domain, or 'coherence lengths', derived from cross-spectra for each frequency. Both representations are considered in the next section for characterizing the spatial evolution of wall-pressure signatures in the streamwise direction.

\subsection{Wall-Pressure Spatial Coherence and Correlations}

This section presents the space-time correlations and cross-spectra computed for microphones aligned in the streamwise direction, up to a maximum separation of $x=4.2 \delta$. From the cross-correlation functions, the correlation length scales and mean convection velocity are computed. The accuracy of existing empirical models for the cross-spectral density and coherence length scales are also investigated. Comparisons with published experimental data are provided to validate the current measurements.

\subsubsection{Streamwise spatial correlations and convection velocity of wall- pressure fluctuations}

The cross-correlation and cross-spectra are computed using measurements from four microphone configurations, as shown in Fig. 4.6. In the configurations shown, measurements were taken with each of the microphones positioned downstream of the central 
microphone, and aligned with the freestream flow. The numbers shown in Fig. 4.6 indicate the order of the microphone in the streamwise array when the panel is rotated. The radial offsets, between linear arrays, allow wall-pressure measurements to be collected with a spacing of $1.6 \mathrm{~mm}$ when rotated by $60^{\circ}$. The last two configurations were not rotated, as cross-correlations and cross-spectra are less sensitive to spatial resolution at large streamwise separations [40]. The spatial separations between each microphone in the array, and the reference microphone (' 1 ' in Fig. 4.6), are listed in Table 4.3 .

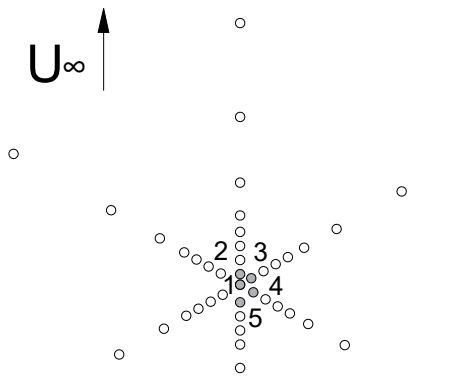

(a)

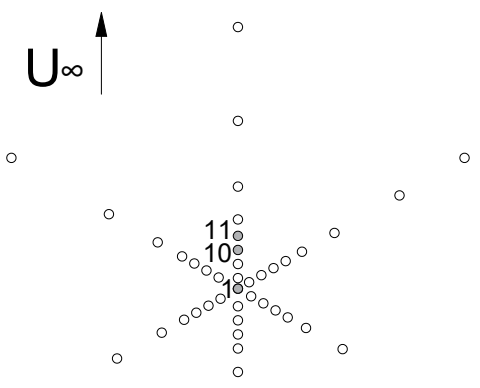

(c)

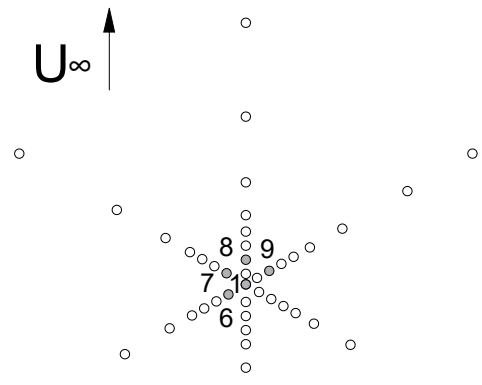

(b)

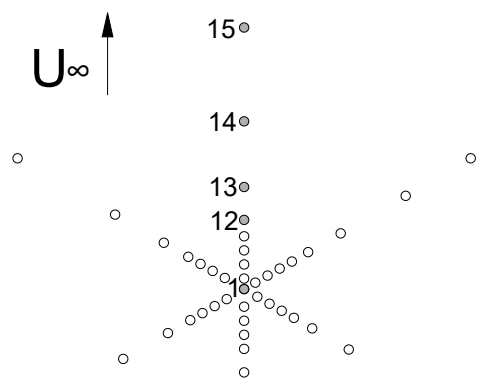

(d)

Figure 4.6: Schematic of microphone arrangements used to measure streamwise crosscorrelations and cross-spectra.

Examples of cross-correlations, at $R e_{\theta}=2,546$, are shown in Fig. 4.7a, The peaks in the cross-correlations are extracted and plotted in isolation, for $R e_{\theta}=1,886$ and $R e_{\theta}=2,546$, in Fig. 4.7b. The measurements presented in Fig. 4.7a were bandpass 


\begin{tabular}{ll|ll}
\hline Microphone & $\xi[\mathrm{m}]$ & Microphone & $\xi[\mathrm{m}]$ \\
\hline 1 & 0.0000 & 9 & 0.0197 \\
2 & 0.0086 & 10 & 0.0276 \\
3 & 0.0102 & 11 & 0.0372 \\
4 & 0.0118 & 12 & 0.0468 \\
5 & 0.0134 & 13 & 0.0657 \\
6 & 0.0149 & 14 & 0.1038 \\
7 & 0.0165 & 15 & 0.1420 \\
8 & 0.0181 & & \\
\hline
\end{tabular}

Table 4.3: Streamwise spacing between each microphone in the array and the reference microphone

filtered, between $55 \mathrm{~Hz}$ and 2,000 Hz, to remove background noise. The crosscorrelation analysis was also performed on the unfiltered data set (not shown), which exhibited similar trends, but with higher scatter and slightly elevated correlations at the largest spatial separations. The most notable difference was the widening of the correlation peaks, in the unfiltered data set, which made peak detection more difficult and unreliable for large spatial separations.

The correlation length is proportional to the decay constant of an exponential fitting to the peaks in the cross-correlations measured at each microphone in the array (Eq. (3.25)). The exponential fitting, represented by the dotted lines in Fig. 4.7b, over-predicts the correlation peaks for $\xi<0.06$, and under-predicts thereafter, similar to the behaviours observed for the in-flight data analyzed by Palumbo [11]. The correlation lengths were calculated to be $0.027 \mathrm{~m}$ and $0.019 \mathrm{~m}$ for $R e_{\theta}=1,886$ and $R e_{\theta}=2,546$, respectively. This suggests that the wall-pressure fluctuations retain their energy for longer at lower flow speeds. This longer correlation length, at $R e_{\theta}=1,886$, is driven by the higher correlation peaks at smaller separations $(\xi \leq 0.03 \mathrm{~m})$. However, at 


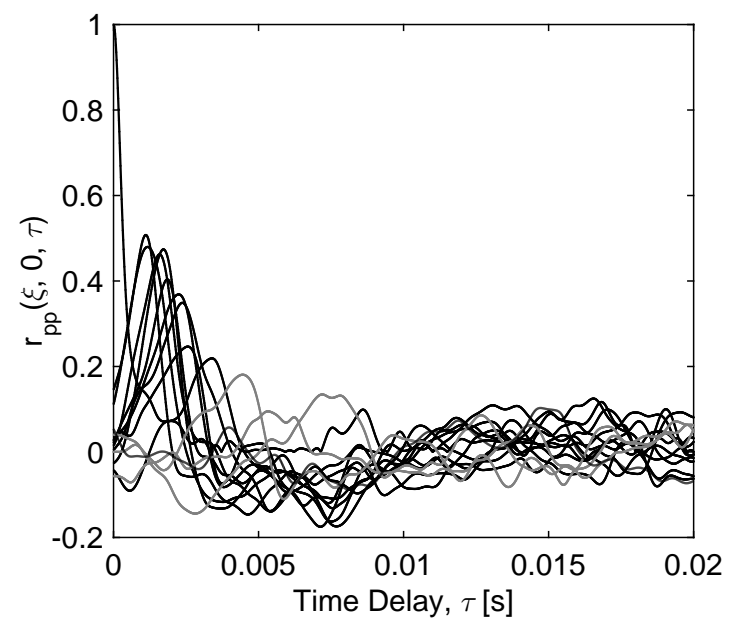

(a)

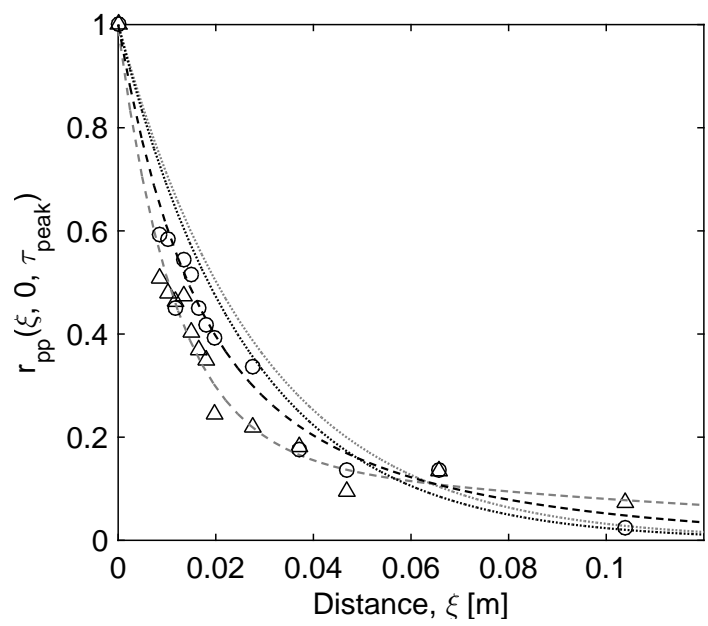

(b)

Figure 4.7: Space-time correlations of wall-pressure fluctuations in the streamwise direction: a) - $\xi / \delta \leq 1.0 ;-, 1.0<\xi / \delta \leq 2.0 ;-. ., \xi / \delta>2.0$ and, b) $\bigcirc, R_{\theta}=1,886$; $\triangle, R e_{\theta}=2,546$; dotted lines $=\exp \frac{-|\xi|}{L_{x}}$; dashed lines $=A \cdot \exp \frac{-|\xi|}{L_{1}}+B \cdot \exp \frac{-|\xi|}{L_{2}}$; black and grey denote $R e_{\theta}=1,886$ and $R e_{\theta}=2,546$, respectively.

larger separations the correlation peaks have lower amplitude. This indicates that the large-scale motions do not remain coherent as long, compared to $\operatorname{Re}_{\theta}=2,546$.

A better fit to the experimental cross-correlation peaks is obtained using the double exponential form proposed by Panton et al. [50] (Eq. (3.26]). These regression fittings are shown in dashed lines in Fig. 4.7b. This form is consistent with the findings of Bull [9], who proposed that the wall-pressure field is comprised of two families of convected structures; one associated with turbulent motions, which are coherent for distances on the order of their wavelength, and the second associated with large-scale outer layer eddies, which decay much slower, and seemingly independent of wavelength.

The model parameters for $R e_{\theta}=1,886$ are: $A=0.62, B=0.38, L_{1}=0.013 \mathrm{~m}$ and $L_{2}=0.050 \mathrm{~m}$, and for $R e_{\theta}=2,546: A=0.78, \quad B=0.22, L_{1}=0.013 \mathrm{~m}$ and $L_{2}=0.157 \mathrm{~m}$. According to Panton et al. [50], the model constants suggest that the single-point spectrum, at $R e_{\theta}=1,886$, receives approximately $62 \%$ of its energy from small-scale structures $(A=0.62)$, and $38 \%$ from large-scale structures $(B=0.38)$. The 
proportions shift slightly, at higher $R e_{\theta}$, with $78 \%$ of the single-point signature contributed by the smaller scale structures. At $R e_{\theta}=2,546$, the correlation length of the large-scale structures also increases by over $310 \%$. These behaviours suggest a change in turbulence structure with increasing $R e_{\theta}$.

Literature shows that, at low Reynolds numbers $\left(R e_{\theta}<5,000\right)$, large-scale motions appear as single hairpin vortices, with little distinction between large and small scales of the flow. At higher $R e_{\theta}$, the difference in length scales are more defined, with the larger structures commonly appearing as quasi-streamwise packets of hairpin vortices [19, 61, 109, 110]. Large zones of uniform momentum, representing the aggregate response of aligned hairpins or packets, may be formed and retained for larger streamwise separations than individual hairpin vortices [19, 111, 112]. This explains the rapid decay in the correlation of smaller structures and sustained coherence at large separations. The signature of individual structures quickly become lost due to growth, turbulent interactions, or viscous diffusion, but the large-scale signature is retained for longer since it is less sensitive to the exact organization of the smaller constituent structures [111].

Further information, regarding the association of wall-pressure cross-correlation features to turbulent motions, can be obtained using the convection velocity, $U_{c}$. The experimental convection velocity, as function of spatial separation, is shown in Fig. 4.8 for both flow conditions. Other measurements from the literature are included for comparison.

The spatial evolution of the convection velocity has the same general form as other measurements from the literature, showing a rapid increase from $U_{c}=0.67 U_{\infty}$, at $\xi=1.6 \delta^{*}$, to a near asymptotic value of $U_{c}=0.82 U_{\infty}$, for $\xi \geq 10 \delta^{*}$. Following from the cross-correlation analysis, this suggests that there are at least two groups of turbulent motions contributing to the wall-pressure signature: motions that decay within 


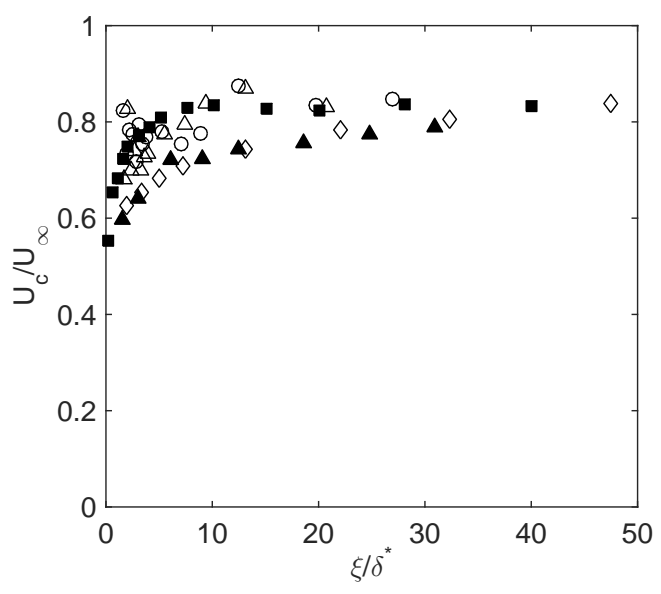

Figure 4.8: Convection velocity derived from cross-correlations: $\bigcirc, R e_{\theta}=1,886 ; \triangle, R e_{\theta}$ $=2,546 ; \mathbf{\square}$, Willmarth \& Wooldridge [7]; $\diamond$, Leclerq \& Bohineust [40]; $\Delta$, Bull [9].

$\xi \geq 10 \delta^{\star}(\xi \leq 50 \mathrm{~mm})$, and those that remain coherent for $\xi>20 \delta^{\star}$.

Using the correlation length scales and the convection velocity, the frequency ranges of these groups can be estimated if it is assumed that the correlation lengths are proportional to the average wavelength of the events. For simplicity, it is assumed that the correlation length scale is constant for all events in each group. For example, the wallpressure fluctuations that retain their energy for $\xi<10 \delta^{*}(0.053 \mathrm{~m})$ are assumed to have a correlation length of $0.013 \mathrm{~m}$ for $R e_{\theta}=1,886$ and $R e_{\theta}=2,546$, and longer-lasting signatures are assumed to have correlation lengths of $0.05 \mathrm{~m}$ and $0.157 \mathrm{~m}$ for $R e_{\theta}=1,886$ and $R e_{\theta}=2,546$, respectively. Average convection velocities, for each of these two groups, are then used to estimate an average frequency. The smaller scale structures $\left(\xi \leq 10 \delta^{*}\right)$ are assumed to convect at $0.72 U_{\infty}$, and larger scale structures $\left(\xi>10 \delta^{\star}\right)$ at $0.82 U_{\infty}$.

For both speeds, the average frequency of the small-scale structures is estimated to be $575 \mathrm{~Hz}$, where as the larger scale pressure fluctuations occur, on average, at $125 \mathrm{~Hz}$ and $54 \mathrm{~Hz}$ for $R e_{\theta}=1,886$ and $R e_{\theta}=2,546$, respectively. This implies that the pressure fluctuations occurring with frequencies near the spectrum peak are responsible for the extended streamwise energy, whereas pressure fluctuations that fall within the overlap 
frequency range decay within $10 \delta^{*}$.

Alternatively, the approach by Bull [9] can be used to roughly locate the events, within the boundary layer, by superimposing the convection velocity onto the mean velocity profile (Fig. 4.3a). This technique suggests that the smaller-scale structures are positioned within $0.10 \leq y / \delta \leq 0.32$, with larger-scale motions originating at $y / \delta \geq 0.32$. These locations correspond to the log-layer, and the upper edge of the log-layer/outer wake, respectively (see Fig. 1.1). This corroborates the notion that the low-frequency structures originate/extend into the outer regions of the boundary layer, and retain their coherence for large distances downstream (up to $3 \delta$ ), whereas high frequency events originate closer to the wall, and lose their coherent energy much quicker [9, 29, 32]. The cross-spectrum analysis will now be considered to study the frequency distribution of the coherent energy between microphones.

\subsubsection{Streamwise cross-spectrum and coherence lengths of wall- pressure fluctuations}

The cross-spectrum provides a visualization of the coherent energy in the wall-pressure signatures, as a function of frequency. Preliminary measurements of cross-spectra, at $R e_{\theta}=1,886$ and $R e_{\theta}=2,593$, from a study by Van Blitterswyk \& Rocha [67], are shown in Fig. $4.9 \mathrm{a}$ and Fig. $4.9 \mathrm{~b}$, respectively. These measurements were collected using the first test panel to investigate the sensitivity of the cross-spectrum to spatial resolution.

Measurements at both speeds show that the coherent power of the low-frequency pressure fluctuations ( $f \leq 200 \mathrm{~Hz}$ ) exceeds the streamwise extent of the microphone array, indicating that these events can retain their signature for at least $3.6 \delta$ in the streamwise direction. The cross-spectrum for $\xi / \delta=0.73$ shows that the energy of pressure fluctuations occurring at $f \geq 700 \mathrm{~Hz}$ cannot be captured using the rectangular microphone 


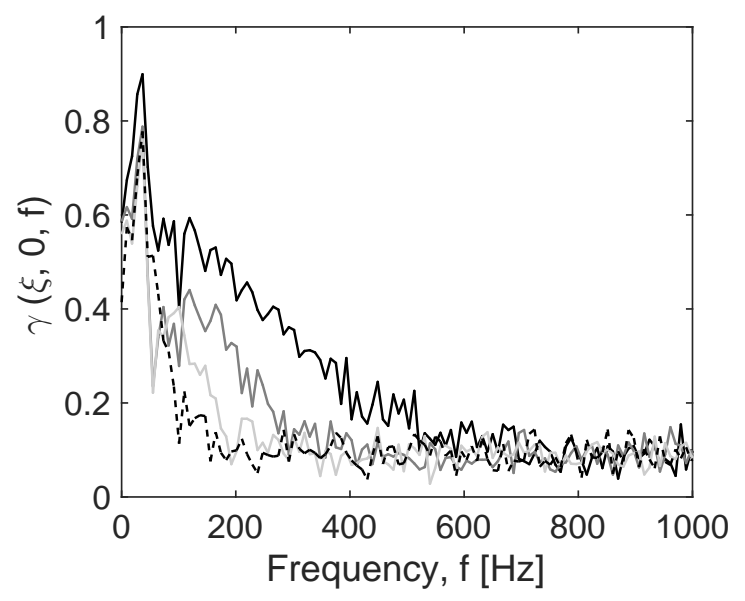

(a)

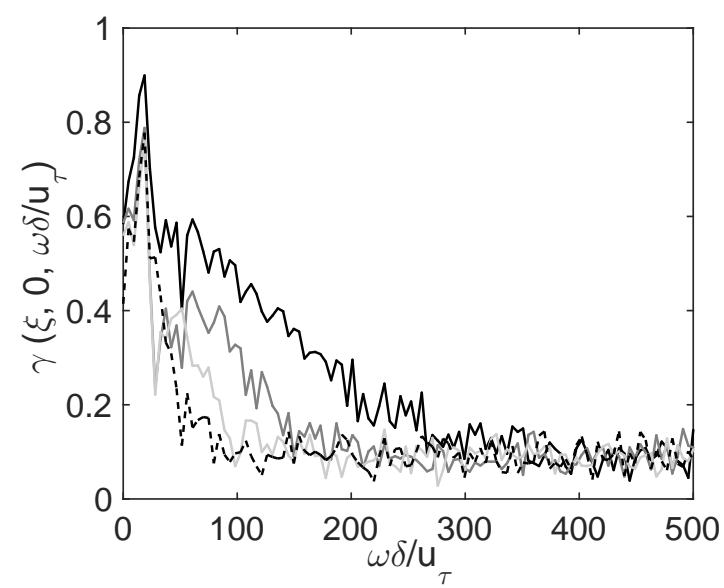

(b)

Figure 4.9: Streamwise cross-spectra between microphones of various streamwise separations as measured for $R e_{\theta}=2,593$ : a) as a function of frequency, and b) as a function of non-dimensional frequency, $\omega \delta / u_{\tau} ;-, \xi / \delta=0.73 ;-, \xi / \delta=1.45$; $\longrightarrow, \xi / \delta=2.54 ;-\cdots, \xi / \delta=3.63$.

array. Based on measurements of the cross-correlation, pressure fluctuations in this frequency range are considered small-scale, and contribute up to $78 \%$ of the single-point spectrum. Therefore, it was concluded that there was a need to develop a new test panel, which would allow proper measurements of the cross-spectrum to be made using with a finer spatial resolution.

Measured cross-spectra, collected using the rotating test panel, with microphones in the configurations shown in Fig. 4.4, are presented in Fig. 4.10a and Fig. 4.10b for the cases of $R e_{\theta}=1,886$ and $R e_{\theta}=2,546$, respectively.

At very low frequencies $\left(\omega \delta / u_{\tau}<30, f<50 \mathrm{~Hz}\right)$, the cross-spectra all exhibit elevated power associated with background noise. Very near to this region is a local peak in coherence, which occurs consistently near $\omega \delta / u_{\tau}=50(f \approx 100 \mathrm{~Hz})$. This second peak corresponds to the peak in the single-point frequency spectrum. The coherence remains well below unity, which is attributed to mean shear effects and the thickness of the boundary layer, which limit the scale and lifetime of coherent structures [40]. 


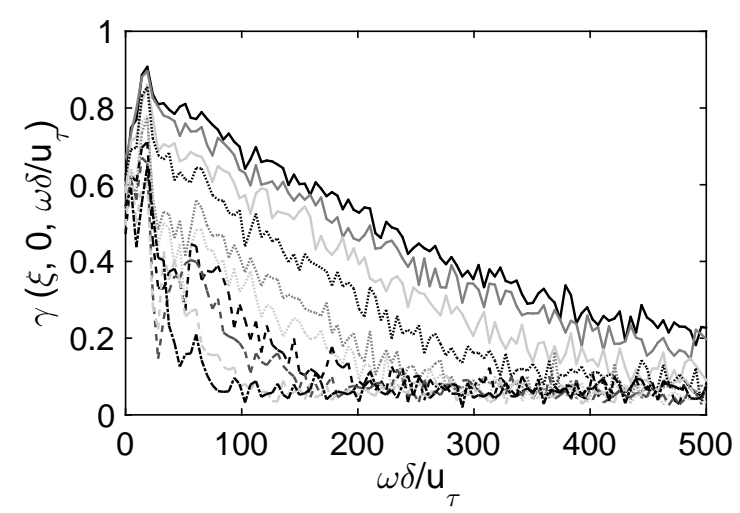

(a)

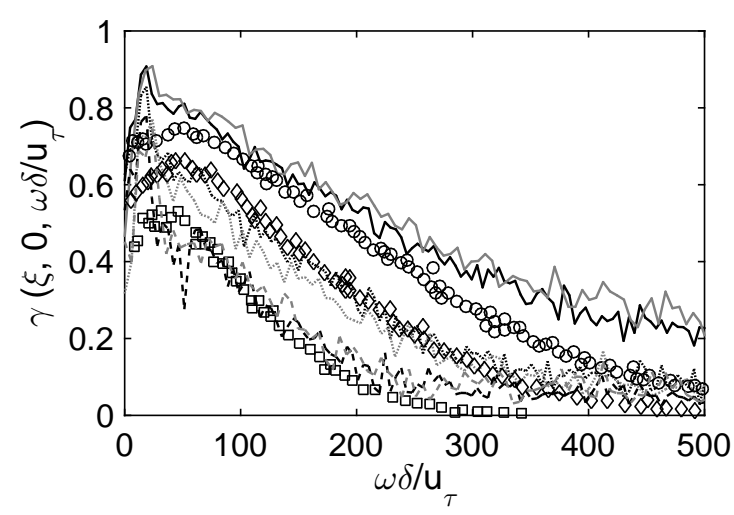

(c)

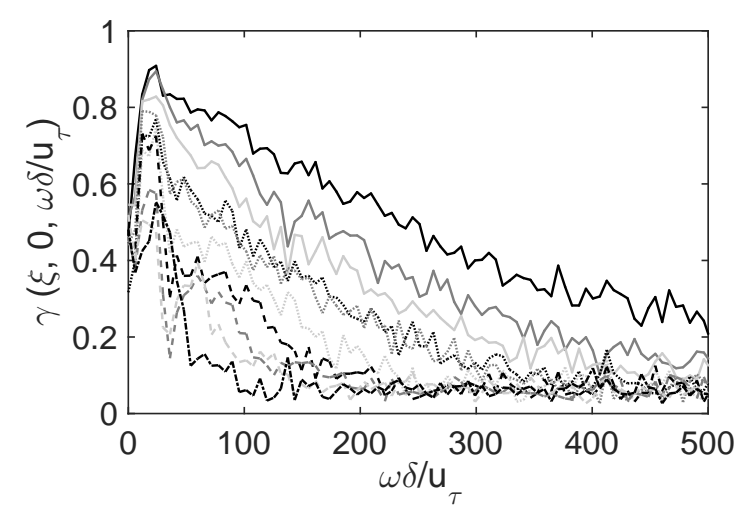

(b)

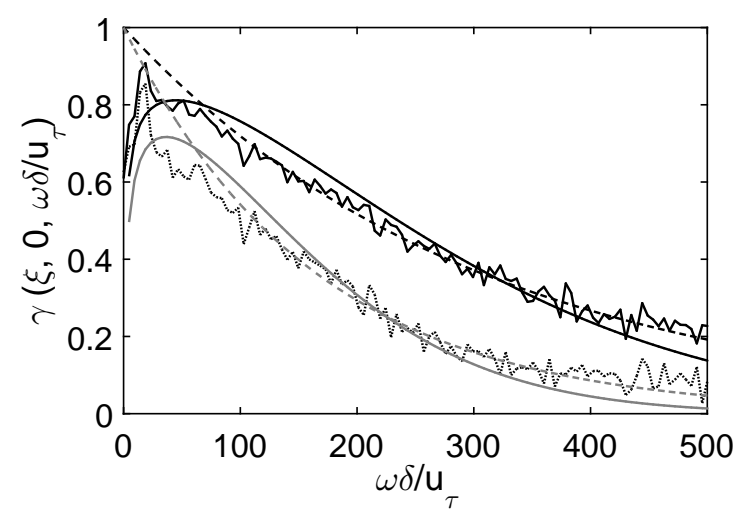

(d)

Figure 4.10: Streamwise cross-spectra between microphones of various streamwise separations: a) $R e_{\theta}=1,886$, b) $R e_{\theta}=2,546$, c) comparison of experimental crossspectra with measurements from Leclerq \& Bohineust [40] at $\xi / \delta=0.24, \xi / \delta=0.42$, and $\xi / \delta=0.90$, and d) comparison between experimental cross-spectra for $\xi / \delta=$ 0.26 and $\xi / \delta=0.53$ and predictions using the models proposed by Corcos [10] (Eq. (3.29) ) (dashed lines) and, Leclerq \& Bohineust [40] (Eq. (3.33)) (solid lines). In a), and b): —, $\xi / \delta=0.26 ;-, \zeta / \delta=0.31 ;-, \zeta / \delta=0.41 ; \cdots, \xi / \delta=0.53 ; \cdots, \xi / \delta=$ $0.85 ; \cdots, \xi / \delta=1.14 ;-.-, \xi / \delta=1.40 ;-=-, \xi / \delta=2.02 ;-\cdots, \xi / \delta=3.20 ;-\cdots, \xi / \delta=4.37$. In c) solid lines, $\xi / \delta=0.26$, dotted lines, $\xi / \delta=0.53$, and dashed lines, $\xi / \delta=0.85$; black and grey denote $R e_{\theta}=1,886$ and $R e_{\theta}=2,546$, respectively.

The coherence near the spectrum peak remains elevated for up to $4 \delta$ in the streamwise direction. Sustained coherence, for several boundary layer thicknesses downstream, was also reported by Leclerq \& Bohineust [40], Farabee \& Casarella [29], and previous measurements by the author [67]. This prolonged coherence is commonly attributed to 
irrotational motions above the boundary layer [40, 68]. Irrotational motions may also contribute to the coherence below $100 \mathrm{~Hz}$, but it is difficult to isolate their contribution from background effects. It is believed that the elevated coherence at $f=100 \mathrm{~Hz}$ may also be due to the signature of quasi streamwise-aligned hairpins in the form of packets, which can retain their coherence for very large distances [19, 20, 111]. This hypothesis is addressed in greater detail later in this chapter.

When the frequency is normalized, the measurements show excellent agreement between the two flow speeds, considered here, for $\xi / \delta=0.26,0.53$ and 0.85 , and measurements by Leclerq \& Bohineust [40], as shown in Fig. 4.10c. The collapse of the highfrequency portions of the cross-spectra implies that the pressure fluctuations decay at a constant rate, proportional to traveling equal fractions of their lengthscale [29]. The cross-spectra presented by Leclerq \& Bohineust [40] and Farabee \& Casarella [29], show a shift in peak cross-spectrum energy to lower frequencies with increasing separation. The expected shift is not as obvious in the current measurements, however, this is likely a result of the variables selected to normalize frequency. The shift is accentuated when normalized using outer-layer scaling $\left(\omega \delta / U_{c}\right)[29]$. The agreement between the current cross-spectra and the measurements by Leclerq \& Bohineust [40], at low frequencies $\left(\omega \delta / u_{\tau}<200\right)$, provides confidence that the expected behaviours are captured.

At the smallest separation, the current measurements show elevated cross-spectrum energy at higher frequencies $\left(\omega \delta / U_{\tau}>250\right)$ compared to the measurements by Leclerq \& Bohineust [40], but closely match at all non-dimensional frequencies considered. The open jet configuration, used by Leclerq \& Bohineust [40], may have effects on the smallscale structure of the flow due to the formation of mean streamwise pressure gradients. Static pressure was also not measured over the test panel; however, the reduced crossspectrum levels at higher frequencies are characteristic of the effects of a mild adverse pressure gradient [60]. Further, integral boundary layer parameters were predicted and 
not measured, which may contribute to some of the discrepancy between the data sets.

Since the work of Corcos [10], it has become common practice to model the spatial coherence between two points, aligned in the streamwise direction, using an exponential function (Eq. 3.29)). The most obvious shortcoming of this model is a prediction of infinite coherence at zero frequency. More recent literature has suggested that a generic exponential decay parameter cannot be selected to represent the entire cross-spectrum [11, 40, 67]. Despite these limitations, very few other models have been proposed to replace the Corcos model. In this work, the model proposed by Leclerq \& Bohineust [40] for the normalized cross-spectrum, is compared against the Corcos model and experimental data, for microphone spacings of $y / \delta=0.26$ and $y / \delta=0.53$, at $\operatorname{Re}_{\theta}=1,886$ in Fig. 4.10d.

Aside from over-predicting the cross-spectrum amplitude at lower frequencies $\left(\omega \delta / u_{\tau}<100\right)$, the Corcos model is a good candidate for predicting the cross-spectrum at both locations. Suitable decay constants for the Corcos model are 0.45 and 0.48 , for spatial separations of $y / \delta=0.25$ and $y / \delta=0.53$, respectively. When plotted against the cross-spectral phase, $\omega \xi / U_{c}$, (not shown), the curves collapse to an approximate exponential curve with a decay constant of 0.22 , assuming a constant convection velocity of $0.72 U_{\infty}$. The decay of the experimental cross-spectra are slightly higher, compared to values reported in the literature of 0.1 [8], $0.145[29]$ and 0.19 [9]. Considering the measured values, combined with reported values in the literature, it appears that the decay rate is dependent on Reynolds number. This hypothesis is supported within the data collected by Farabee \& Casarella [29], which show that the decay rate increases from 0.125 at $R e_{\theta}=6,025$, to 0.145 at $R e_{\theta}=3,386$.

The model by Leclerq \& Bohineust [40], with the original model parameters, captures the peak in the cross-spectrum around $\omega \delta / u_{\tau}=50$, but slightly over-predicts the crossspectrum amplitudes for $\omega \delta / u_{\tau} \leq 200$, and under-predicts at higher frequencies. This 
behaviour is similar to the comparisons within the study by Leclerq \& Bohineust [40]. This may be a function of the normalization used in the development of their model $\left(\left(f^{2} v \xi^{2}\right)^{1 / 2} / u_{\tau}^{2}\right)$, which does not collapse the data as well as the Corcos similarity scaling for the current measurements. Beyond $\omega \delta / u_{\tau}=500$, the cross-spectrum is underpredicted by both models; however, the asymptotic nature, in this frequency range, is believed to be statistical scatter due to low wall-pressure energy and leakage from window averaging. Therefore, the attempts to assess the accuracy of empirical models, in this frequency range, are not meaningful.

Continuing with the exponential form for modeling the cross-spectrum, one can define a coherence length (Eq. (3.31) ) using cross-spectra collected over a range of spatial separations. For each frequency an exponential function is fitted to the spatial set of cross-spectrum amplitudes. An example of this is shown in Fig. 4.11a for $f=82 \mathrm{~Hz}$. This process is repeated for all frequencies, to obtain the coherence length frequency distribution, as shown in Fig. 4.11b. Re-processed measurements from Van Blitterswyk \& Rocha [67] at $R e_{\theta}=2,593$ are also provided to compare measurements collected using the two test panels.

From Fig. $4.11 \mathrm{~b}$, it is observed that the maximum coherence length, and peak frequency increase with increasing $R e_{\theta}$. At $R e_{\theta}=1,886$ the maximum coherence length is $55 \mathrm{~mm}$ with a corresponding frequency of approximately $92 \mathrm{~Hz}$. The maximum coherence length increases to $82 \mathrm{~mm}$, at $R e_{\theta}=2,546$, with a corresponding peak frequency of $119 \mathrm{~Hz}$. The peak frequency occurs very near the peak in the single-point wallpressure spectrum for both speeds (Fig. 4.4). The coherence length distributions show that high-frequency fluctuations retain their coherence for greater streamwise distances with increasing $R e_{\theta}$. This offers support for the postulation, based on cross-correlation measurements, of a shift in coherent structures to smaller-scales with increasing $R e_{\theta}$. At 


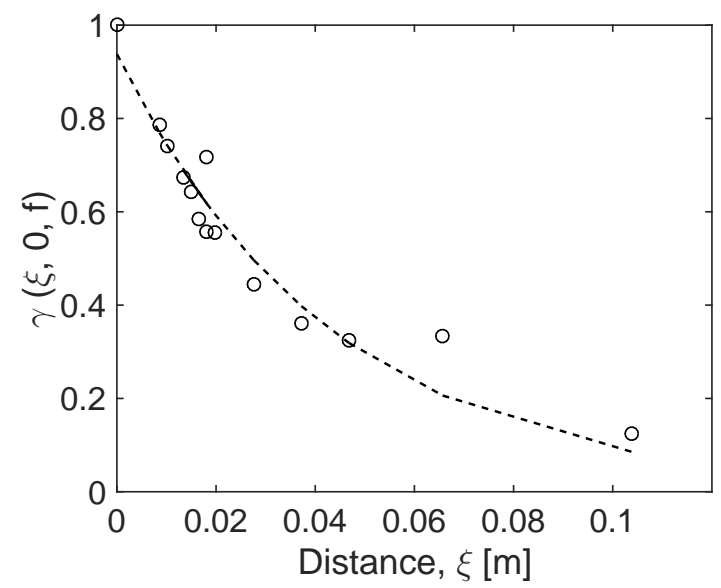

(a)

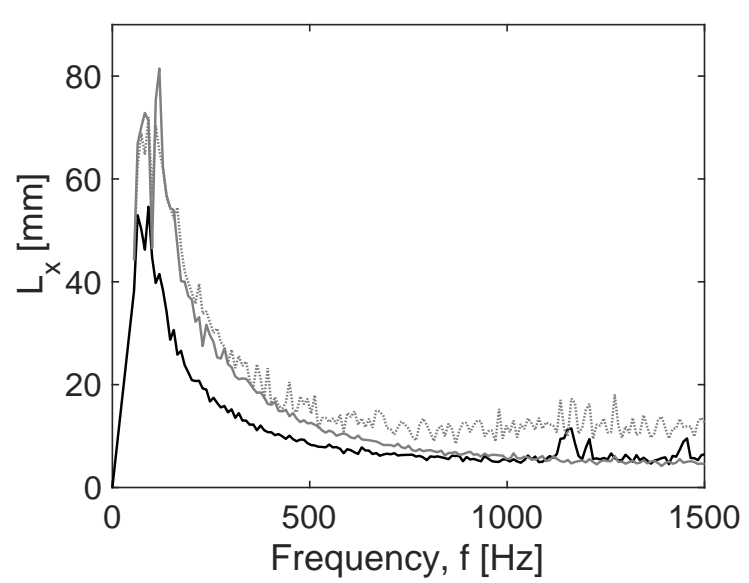

(b)

Figure 4.11: Coherence lengths analysis for $R e_{\theta}=1,886, R e_{\theta}=2,549$ and $R e_{\theta}=2,593$ from Van Blitterswyk \& Rocha [67]: a) an example of the normalized crossspectrum amplitude as measured by each microphone in the rotating array, used to derive the coherence length at $82 \mathrm{~Hz}$ for $R e_{\theta}=1,886$, and b) coherence lengths for all frequencies up to $1,500 \mathrm{~Hz} ;-, R e_{\theta}=1,886 ;-, R e_{\theta}-2,546 ; \ldots ., R e_{\theta}=2,593$. The dashed line in a) denotes the exponential fitting used to extract the coherence length.

higher $R e_{\theta}$, these smaller-scale structures have higher energy, and may travel in groups, or packets, which likely explains the extended spatial coherence.

The coherence length eventually reaches an asymptotic value for $f \geq 750 \mathrm{~Hz}$ and $f \geq 1,000 \mathrm{~Hz}$ at $R e_{\theta}=1,886$ and $R e_{\theta}=2,546$, respectively. The exponential form, used to define the coherence length, is responsible for the asymptotic behaviour at highfrequencies. While this form holds well for frequencies near the single-point spectrum peak, as demonstrated in Fig. 4.11a it does not hold well at higher frequencies, where the cross-spectrum amplitudes are very low and nearly constant between microphones (Fig. 4.10a and Fig. 4.10b). It is interesting to note that the general trends can be captured using the non-rotating test panel; however, the coherence lengths are slightly elevated above the peak frequency, and reaches an asymptotic value earlier $(f \geq 500 \mathrm{~Hz})$, due to fewer data points available to derive the exponential fitting. 
The models proposed by Corcos [10] (Eq. (3.31) and Efimtsov [52] (Eq. [3.32), for predicting the coherence length distribution, are compared against experimental measurements in Fig. 4.12, The experimentally-derived empirical constants for each model, along with those reported in the literature, are given in Table 4.4 .

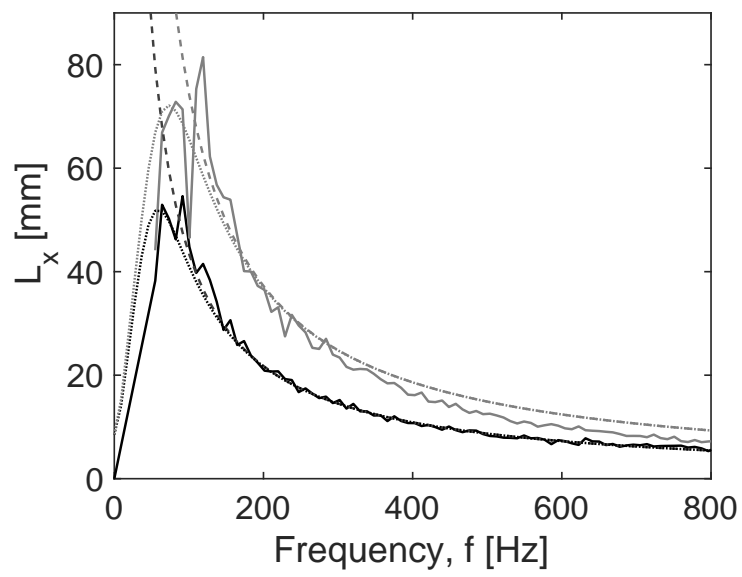

Figure 4.12: Comparison of measured coherence length distributions to empirical models proposed by Corcos [10] (Eq. (3.31)) and Efimtsov [52] (Eq. (3.32)): - $R e_{\theta}=$ 1,$886 ;-, R e_{\theta}=2,546$. Dashed and dotted lines denote predictions with the Corcos model and Efimtsov model, respectively.

\begin{tabular}{llll}
\hline Data Set & $a_{1}=\alpha_{x}$ & $a_{2}$ & $a_{3}$ \\
\hline$U_{\infty}=7.6 \mathrm{~ms}^{-1}$ & 0.20 & 18.0 & 4.0 \\
$U_{\infty}=10.4 \mathrm{~ms}^{-1}$ & 0.16 & 12.5 & 4.0 \\
$U_{\infty}=225 \mathrm{~m} / \mathrm{s}[52]$ & 0.10 & 72.8 & 1.54 \\
$U_{\infty}=174 \mathrm{~m} / \mathrm{s}$ [1] & 0.06 & 5.0 & 1.0 \\
\hline
\end{tabular}

Table 4.4: Coherence length parameters reported in the literature and derived from experimental measurements

A qualitative comparison shows that the Corcos model is in excellent agreement with experimental measurements beyond the peak frequency at $R e_{\theta}=1,886$. The Efimtsov model improves the prediction of the Corcos model by capturing the peak in coherence 
lengths around $92 \mathrm{~Hz}$. The coherence length distribution at $R e_{\theta}=2,546$ is not modeled as well using an exponential form, suggesting a Reynolds number dependence on its shape. The models were calibrated to match the experimental coherence length values near the peak frequency, which consequently results in an over-prediction at higher frequencies $(f \geq 285 \mathrm{~Hz})$. The Efimtsov model captures the peak in coherence length near $119 \mathrm{~Hz}$ reasonably well, but does not reduce the high-frequency over-prediction.

The coherence length parameters, derived from experimental measurements, show a Reynolds number dependence for $a_{1}$ and $a_{2}$, but due to contamination of the signal, the Reynolds dependence could not be assessed for the low-frequency roll-off $\left(a_{3}\right)$. Comparison with parameters used in the literature (Table 4.4) are not expected to be in agreement due to the vast differences in Reynolds number. However, the trends in values confirm the suggested Reynolds number dependencies from the current measurements, showing that the coherence length decay becomes more gradual at higher Reynolds numbers (based on decreasing $a_{1}$ ), and the peak coherence length continues to increase (based on decreasing $a_{2}$ ). The non-exponential decay, measured at $R e_{\theta}=2,546$, has a similar form to in-flight measurements collected by Palumbo [11], suggesting that the boundary layer, and its associated time-averaged wall-pressure signatures, may be approaching a self-similar state to high Reynolds number flows.

\subsection{Wall-Pressure - Velocity Correlations}

This section further examines the relationship between turbulent structures inferred from wall-pressure signatures, using the normalized cross-spectrum between velocity and pressure fluctuations. The cross-spectra between streamwise velocity fluctuations and wall-pressure fluctuations, at various positions within the boundary layer, are shown in Fig. 4.13. The measurements presented are not filtered for background noise 
to show its relative coherence amplitude compared to velocity fluctuations within the boundary layer. That said, the following discussions will only focus on features above the background noise cut-off frequency of $55 \mathrm{~Hz}$.

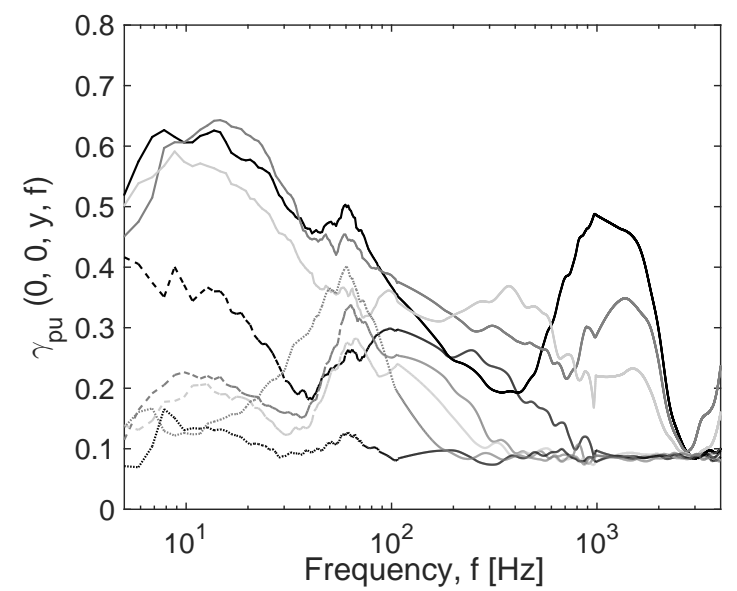

(a)

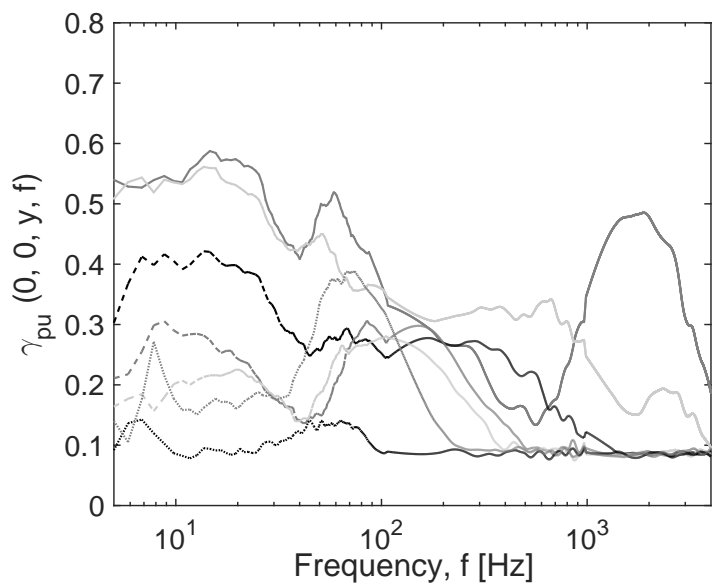

(b)

Figure 4.13: Cross-spectrum between streamwise velocity fluctuations and pressure fluctuations measured at different regions in the boundary layer at $x=1.10 \mathrm{~m}$ : a) $R e_{\theta}=1,886$, and b) $R e_{\theta}=2,546$. - $y / \delta=0.007 ;-, y / \delta=0.010 ;-, y / \delta=$ $0.019 ;-\cdots, y / \delta=0.067 ;--=, y / \delta=0.17 ;-\cdots, y / \delta=0.24 ; \cdots ., y / \delta=0.74 ; \cdots, y / \delta=$ 1.40 .

For all speeds, the cross-spectrum within the buffer layer $(y / \delta \leq 0.019)$ exhibits a double-humped structure, which is in good agreement with other measurements for low Reynolds number flows over flat plates and cylinders [68, 74]. The first peak exists at approximately $60 \mathrm{~Hz}$, and the second around 1,000 $\mathrm{H}$ for $\operatorname{Re}_{\theta}=1,886$. The location of these peaks shift slightly, to higher frequencies, at $R e_{\theta}=2,546$. The cross-spectrum energy is locally elevated at wall-normal positions above the buffer layer $(0.019 \leq y / \delta \leq$ 0.24), around $100 \mathrm{~Hz}$ (Fig. 4.13a), corresponding to the peak in the single-point spectrum (Fig. 4.4). Turbulent activity in this region of the boundary layer generates high Reynolds stresses, which is typically associated with the formation of shear layers, via induced ejections from hairpin vortices [23]. The accompanying peaks, at $60 \mathrm{~Hz}$ and 
$1,000 \mathrm{~Hz}$, suggest that the activity in the buffer layer is associated with the formation of large-scale and small-scale structures, much like that expected from several aligned hairpins, as concluded using from cross-correlation analysis (section 4.4.1).

Further away from the wall, the cross-spectrum energy decreases and the highfrequency peak rapidly disappears, supporting the idea that near-wall events primarily influence the behaviour of the high-frequency spectrum. In the logarithmic region of the boundary layer $(0.067 \leq y / \delta \leq 0.24)$, the cross-spectrum is represented by a broad peak that narrows and shifts to lower frequencies with increasing distance from the wall. The effects of small-scale structures are not felt in the outer regions of the boundary layer, which explains the decrease in energy at high-frequencies, with increasing distance from the wall. Velocity fluctuations in this region all contribute energy to the overlap range of the single-point wall-pressure spectrum. With increasing probe distance from the wall the cross-spectrum decreases to a single peak around $60 \mathrm{~Hz}$ at $y / \delta=1.43$. This is consistent with the notion of irrotational motion above the boundary layer, as proposed by Bradshaw [113]. This peak also overlaps with the first peak observed near the wall, suggesting that the large scale motions span the entire boundary layer, or a related to large-scale structures originating in the inner layer.

\subsection{Coherent Event Detection Using a Wavelet Transform of Wall-Pressure Fluctuations}

Standard time-averaged correlation techniques, and Fourier-based cross-spectrum analyses, play an important role in understanding the aggregate characteristics of the wall-pressure signatures; however, temporal correlations provide no information about the space scale, and Fourier-based approaches lose all frequency locality in the signal, making it difficult to relate characteristics of the spectrum to physical features in the 
flow [12]. Therefore, the approach now shifts to using time-dependent analyses, based on the wavelet transform, to detect and study the underlying organization of coherent events contributing to measured wall-pressure fluctuations.

The wavelet transform enables one to visualize the distribution of energy, as a function of scale and time [12]. Analyses using the wavelet transform have been successfully applied to track coherent structures in turbulent flows using velocity measurements [13[16], and wall-pressure signatures [17, 18]. In this section, wavelet transforms of measured pressure signals are statistically analyzed, using wavelet cross-correlations [16], and higher-order statistics to identify, and track coherent turbulent motions, using arrays of streamwise microphones (Fig. 4.14). These analyses provide supplemental information to explain average statistical properties obtained using the cross-correlation and cross-spectrum analyses.

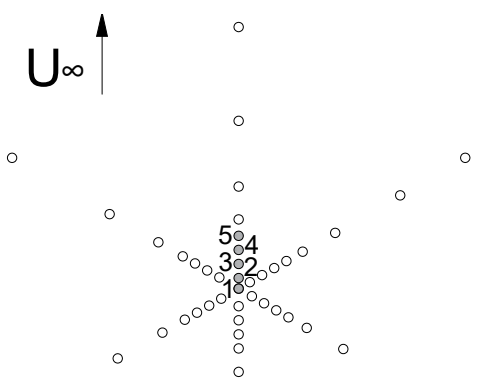

(a)

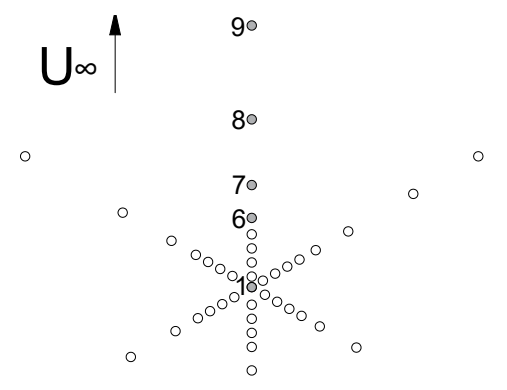

(b)

Figure 4.14: Streamwise microphone arrays used to characterize: a) the convective nature of higher frequency events (Type 4, 5 and 6 events), and b) the convective nature of lower frequency events (Type 1, 2 and 3 events) and event packets.

Similar to the Fourier transform, combinations of the mother wavelet are used to decompose the pressure signal into different scales, each with varying amplitudes. The scale information is contained within the wavelet coefficients. Scales with high amplitude are considered to be the dominant contributor to the wall-pressure signature, and are used to localize events as a function of time. Regions of high amplitude are tracked 
by correlating energy in the wavelet coefficients, between microphones in the array, to infer properties about the turbulent structures that contribute to the coherent energy. An example of a wavelet cross-correlation plot, between microphones with a streamwise spacing of $0.25 \delta$ (between microphones 1 and 2 in Fig. 4.14), is shown in Fig. 4.15a. This is compared to the traditional cross-correlation relationship, between the same microphones, in Fig. 4.15b.

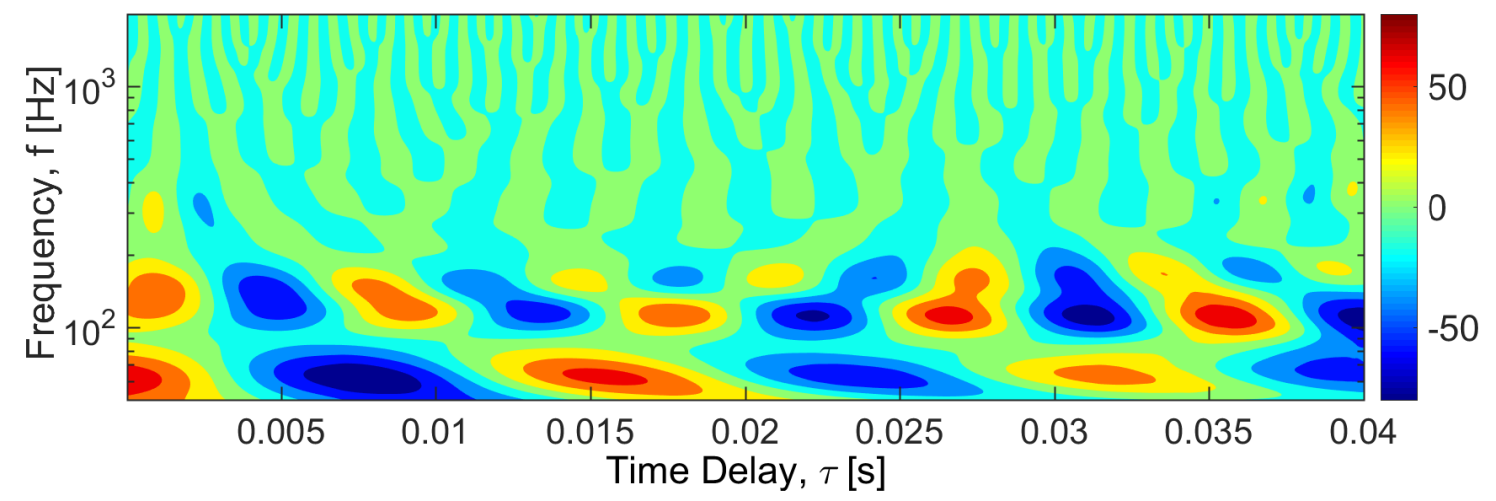

(a)

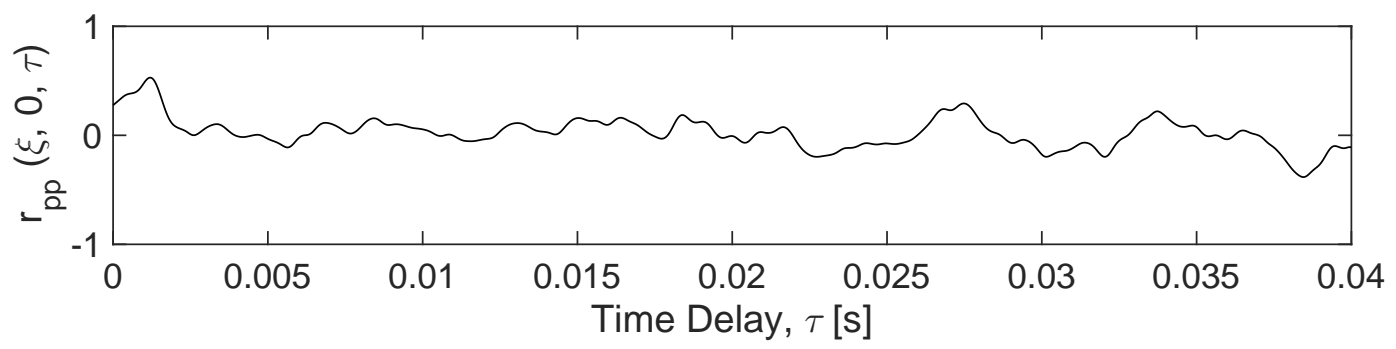

(b)

Figure 4.15: Wavelet cross-correlation analysis of wall-pressure fluctuations measured by two microphones with a streamwise separation of $0.25 \delta$, under conditions of $R e_{\theta}=2,546$ : a) real part of wavelet cross-correlation coefficients, $w_{12}(s, \tau)$ (Eq. (3.36) ), and b) traditional time-averaged cross-correlation, $r_{p p}(\xi, 0, \tau)$, as defined by Eq. (3.9).

The $w_{12}(s, \tau)$ distribution shows that the wall-pressure exhibits alternating, strong positive and negative correlation peaks around $f=60 \mathrm{~Hz}$, each of which is associated with two peaks around $f=120 \mathrm{~Hz}-200 \mathrm{~Hz}$. Some of these events are further related 
to pairs of events, occurring with a frequency of approximately $f=300 \mathrm{~Hz}-400 \mathrm{~Hz}$, or in some instances, events in the range of $f=500 \mathrm{~Hz}-1,000 \mathrm{~Hz}$; however, these peaks occurred less than $10 \%$ of the time. The contributions at high frequencies are likely masked by high energy, larger-scale signatures that influence the near-wall motions [57]. Although not shown by the measurements in Fig. 4.15a, the periodicity in the wavelet cross-correlation is preserved for at least $1 \delta$. The extent of the streamwise correlation, and preserved organization of multi-scale energy in $w_{12}(s, \tau)$, across a range of frequencies, is characteristic of quasi-streamwise hairpin vortex packets [19].

To make more convincing claims about the relationship between pressure and turbulent events, an effort was made to extract information about individual events from the wavelet cross-correlation. Two frequency-dependent thresholds, based on the energy in $w_{12}(s, \tau)$, were employed to localize events in time signals. This approach was inspired by the work of Camussi et al. [17]; however, the thresholds were adjusted from their work to obtain a reasonable compromise between a high correlation level, and event sample size for statistical analyses. In this work, the thresholds were set at 0.8 and 0.5 , of the maximum cross-correlation energy in $w_{12}(s, \tau)$, for lower frequencies $(f<250 \mathrm{~Hz})$ and higher frequencies, respectively. The beginning and duration of events, with localized correlation exceeding the threshold level, were then extracted for further analysis.

The PDF of the time delay between successive events was used to evaluate statistical independence. Sample PDFs for two different microphone locations, and flow speeds, are presented in Fig. 4.16, along with the statistics from experimental data presented by Camussi et al. [17] for comparison. Statistics presented in Fig. 4.16 exhibit an exponential PDF distribution, as expected for uncorrelated events, which confirms that the detected events are not influenced by background effects [17]. It also suggests that detected events are related to coherent motions in the turbulent boundary layer [13, 17]. 


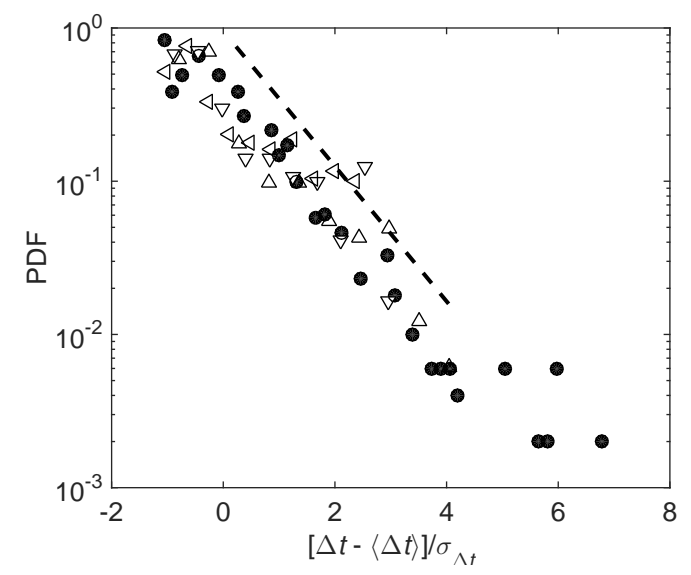

Figure 4.16: Probability density function of time delay between successive events for various flow conditions and microphone placements: $\mathbf{m}$, Camussi et al. [17]; $\triangle$, $R e_{\theta}=2,546-\xi=8.6 \mathrm{~mm} ; \nabla, R e_{\theta}=2,546-\xi=18 \mathrm{~mm}, \triangleleft, R e_{\theta}=1,886-\xi=8.6 \mathrm{~mm}$; $\triangleright, R e_{\theta}=1,886-\xi=18.6 \mathrm{~mm}$. Dashed line denotes a pure exponential decay.

Palumbo [11] found that the shape of the streamwise correlation for individual events is more rectangular, unlike the time-averaged exponential decay when considering the entire signal. This was investigated for a number of events in the overlap frequency range of the single-point spectrum $(f=125-500 \mathrm{~Hz})$, as identified in $w_{12}(s, \tau)$, at $R e_{\theta}=2,546$. For each event, and microphone in the array, an ensemble of data was extracted starting at the detection trigger time from $w_{12}(s, \tau)$. These ensembles were analyzed by computing normalized cross-correlations across the microphones in the array to determine the convective properties of these events. The crosscorrelation peaks and convection velocity for eight events are presented in Fig. 4.17a and Fig. 4.17b respectively for $f=125-250 \mathrm{~Hz}$, and Fig. $4.17 \mathrm{c}$ and Fig. $4.17 \mathrm{~d}$, respectively for $f=250-500 \mathrm{~Hz}$.

From the correlation plots in Fig. $4.17 \mathrm{a}$ and Fig. $4.17 \mathrm{c}$, the rectangular correlation distribution, reported by Palumbo [11], does not seem apparent for all events. The 


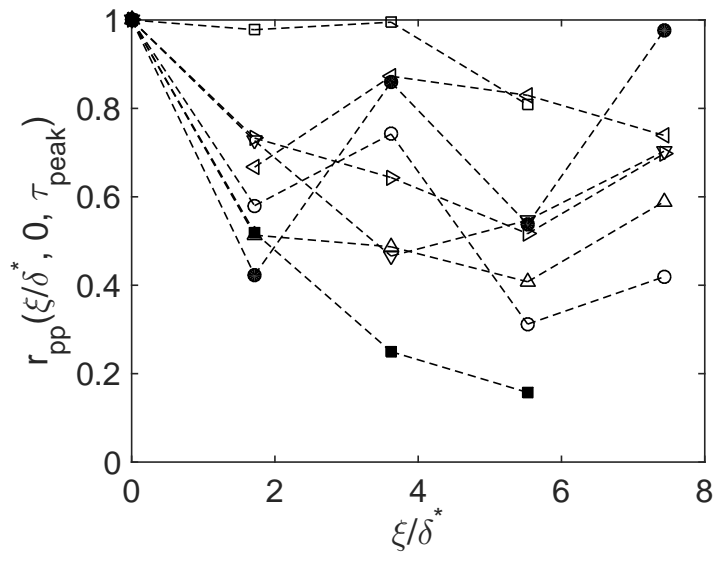

(a)

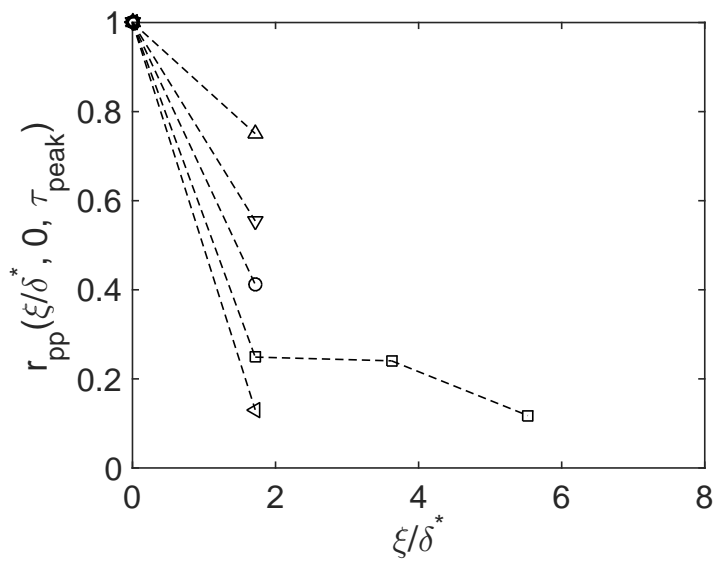

(c)

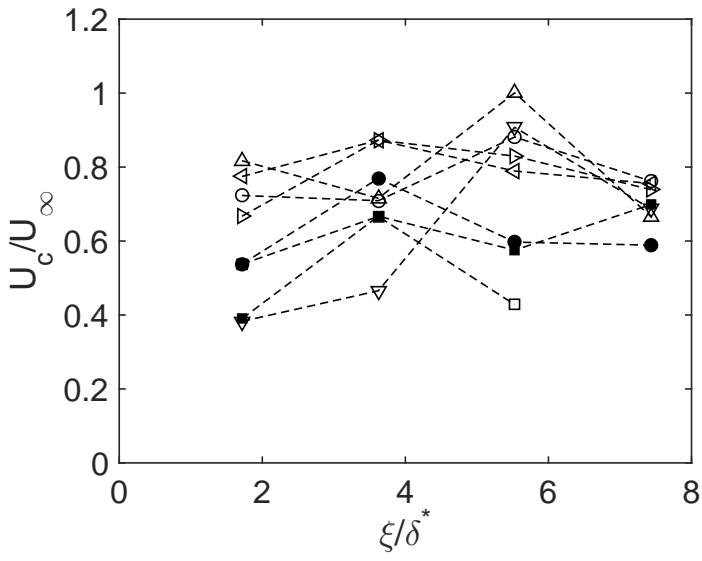

(b)

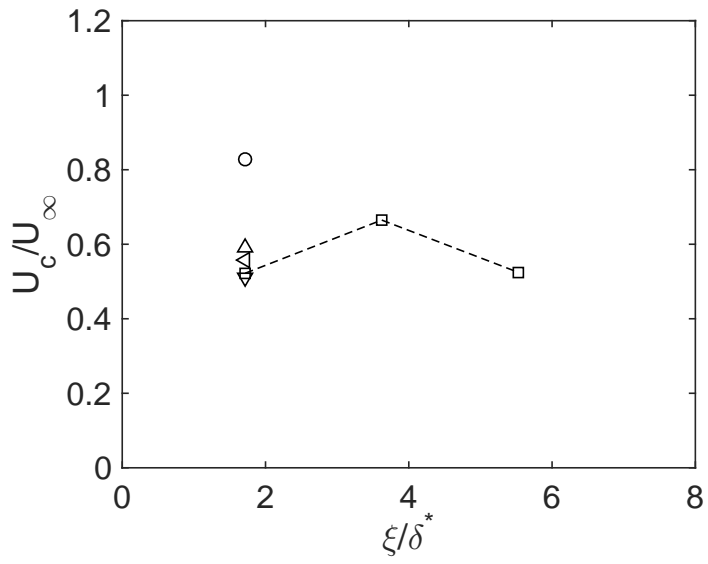

(d)

Figure 4.17: Streamwise cross-correlation, $r_{p p}(\xi, 0, \tau)$, and normalized convection velocity, $U_{c}$, of events detected using the wavelet cross-correlation analysis for $R e_{\theta}$ $=2,546$ : a) $r_{p p}(\xi, 0, \tau)$, and b) $U_{c}$ for events in the $125-250 \mathrm{~Hz}$ frequency band, c) $r_{p p}(\xi, 0, \tau)$, and d) $U_{c}$ for events in the $250-500 \mathrm{~Hz}$ frequency band. Dashed lines in place to emphasize trends.

streamwise decay of energy for events, in the $125-250 \mathrm{~Hz}$ frequency range, is very sporadic, but is not exponential. The majority of event have sustained correlations up to $x=7.5 \delta^{*}$. Events in the $250-500 \mathrm{~Hz}$ frequency band also have high scatter but, in all cases, show a rapid decay in energy before $x=1.7 \delta^{*}$.

The discrepancy in decay behaviours between the frequency bands support the findings from time-average cross-correlations that smaller-scale 
structures drive the shape of the spectrum at small separations, with largerscale structures primarily influencing correlations at large separations. Further, it is noted that in many cases the correlations in Fig. $4.17 \mathrm{a}$ rises at $x=7.5 \delta^{*}$ following a decrease in correlation upstream. This is believed to be a result of the formation of a new event; however, this is difficult to confirm due to the time-averaged nature of the cross-correlation analysis. If it is assumed that this local minimum can be used to define the end of an event, it is estimated that events vary in length from 775 viscous units, in the $125-250 \mathrm{~Hz}$ band, to 240 viscous units in the $250-500 \mathrm{~Hz}$ band. The length of events in the $250-500 \mathrm{~Hz}$ band is typical of individual hairpin vortices, as reported in the literature (100-300 viscous units) [19, 20, 112].

The convection velocity of events in the $125-250 \mathrm{~Hz}$ frequency band (Fig. 4.17b show significant scatter, but a general trend of increasing velocity with increasing spatial separation. The spatial variations in convection velocity of events in the 250-500 Hz frequency band (Fig. 4.17d) are difficult to discern due to the shorter lifetimes. The lower frequency events appear to convect faster as they progress downstream, suggesting these events are located further from the wall. Assuming an average convection velocity of $U_{c}=0.7 U_{\infty}$, based on Fig. 4.17b, and an average duration of $0.005 \mathrm{~s}$, based on the average duration of positive correlation peaks in $w_{12}(s, \tau)$, events in the $125-250 \mathrm{~Hz}$ band have an approximate length of 1,000 viscous units. This is characteristic of the lengths of hairpin packets detected by Adrian et al.[19] and the relationship with smaller scale correlation peaks, at higher frequencies, suggests that the lowfrequency events represent the aggregate signature of several smaller, aligned events.

The wavelet cross-correlation shows concentrated and coherent energy at frequencies below $250 \mathrm{~Hz}$. Therefore, their contributions to the wall-pressure statistics will be assessed using conditional ensemble averages. Based on the detection of a positive, lowfrequency correlation peak, a window of pressure is extracted (750 samples in length), 
representing the average time delay between consecutive positive and negative correlation peaks in this frequency range. This process was repeated for the entire data set (approximately $6 \times 10^{5}$ points). The conditionally sampled cross-spectrum is compared to the standard Fourier-based approach, at two different locations and two different speeds in Fig. 4.18 .

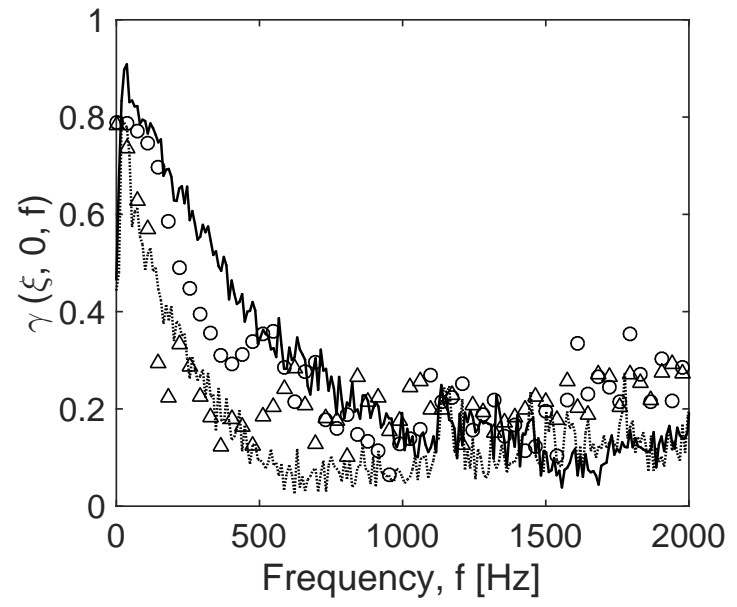

(a)

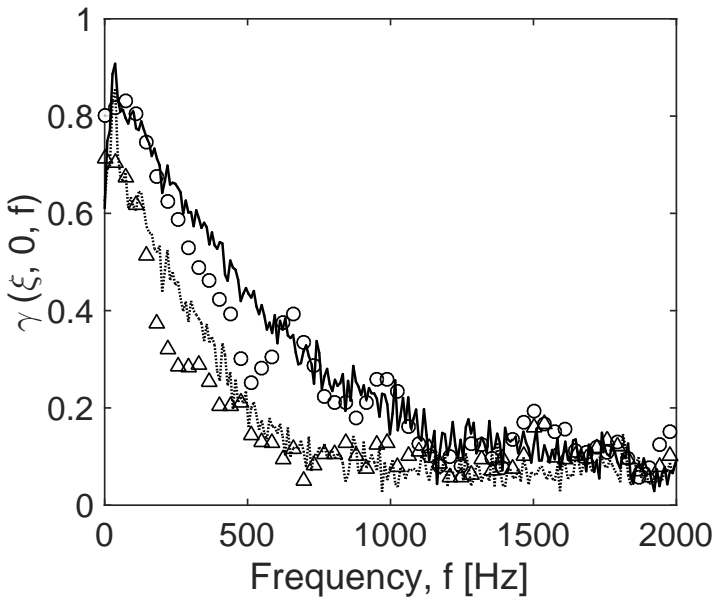

(b)

Figure 4.18: Fourier-based cross-spectrum $(-, \xi / \delta=0.25, \cdots . ., \xi / \delta=0.53)$ compared with the conditional sampling counterparts (open symbols): a) $R e_{\theta}=1,886$, and b) $R e_{\theta}=2,546$.

It is generally observed that the cross-spectra are in good agreement, and accurately capture the coherence peak. The conditionally-sampled cross-spectra also filter out the low-frequency background noise, as the wavelet scales were selectively chosen to omit frequencies below $55 \mathrm{~Hz}$. Fig. 4.18 highlights an important characteristic of the wallpressure fluctuations in that the majority of events contributing to the Fourier-based coherence are concentrated within intermittent, low-frequency, large-scale motions. This supports the findings of Camussi et al. [17] and has important implications for theoretical modeling of TBL-induced wall-pressure excitations. This is characteristic of 
the large-scale coherent motions induced by hairpin packets, and supports the findings from the wall-pressure-velocity cross-spectrum analysis (section 4.5. Therefore, this analysis suggests that pressure fluctuations, occurring below $250 \mathrm{~Hz}$, are representative of the aggregate signature from hairpin vortices organized in quasi-streamwise packets.

From this, it follows that the pressure fluctuations, occurring with frequencies above $250 \mathrm{~Hz}$, are likely associated with smaller, coherent structures, which contribute to the hairpin packet signature. The wavelet cross-correlation analysis is not as efficient at localizing these smaller-scale structures, when detection relies on correlation amplitudes, as these structures have lower energy, and shorter lifetimes. This reduces the likelihood of detection among larger-scale motions in time-averaged analyses. This is especially true for low Reynolds number TBLs, where large scale motions can span much of the boundary layer, and have significant influence on inner-layer motions [56, 57]. Therefore, a more robust approach is required to capture the contribution of these events.

\subsubsection{Event detection using higher-order statistics}

Previous studies have shown that the multi-scale nature of coherent turbulent structures may be studied using the intermittency of velocity signals [14, 15, 84]; however, the relationships have yet to be fully clarified. To the authors' knowledge, this has also yet to be studied using wall-pressure fluctuations. Therefore, the applicability of this approach will be tested on wavelet transformations of five ensembles of data, for each flow speed, in an effort to identify, localize and track coherent structures, using wall-pressure measurements.

The energy in the wall-pressure signatures, measured at each microphone in the array, is first described using the wavelet transformation (Eq. 3.34). Kurtosis, $K$ 
(Eq. (3.5)), of the energy in the wavelet coefficients, over all scales, is then used to identify periods in the pressure signal with high activity. The kurtosis describes the relative weight in the tails of the probability distribution with high kurtosis indicating intermittent behaviour in the signal [37]. Events are localized as periods in the pressure ensemble that exhibit kurtosis values exceeding the Gaussian noise threshold $(K=3)$, as employed by Yang \& Nan [15]. Intermittency is a more robust approach to detecting lower-energy structures [14], compared to cross-correlation amplitude, as employed by other studies [16, 17] for detection of large, high energy coherent structures.

The same microphone arrays (Fig. 4.14a and Fig. 4.14b) were used to study the organization and convective nature of small-scale and large-scale motions, respectively. Events detected in the first microphone of the array are tracked in the kurtosis signatures computed for all other microphones in the array. The known spatial separation and time delay between adjacent microphones is then used to define the convection velocity and length of each detected event. The wavelet coefficients were sub-divided into frequency bands, to study the multi-scale organization and composition of the large-scale events identified in the wavelet cross-correlation analysis. The frequency bands were selected based on the approximate extents of regions in the single point power spectrum where energy follows a power law dependence on frequency. The designations assigned to events identified in each band are listed in Table 4.5 .

To confirm the statistical independence of the detected events, the PDF of the time delay between consecutive events is shown in Fig. 4.19, for both flow speeds, with the statistics from experimental data from Camussi et al. [17] presented for comparison. The PDF of the time delay is again, well-characterized by an exponential distribution, and with less scatter compared to the cross-correlation approach. The physical interpretation, and properties of the identified events will be discussed in the next section. 


\begin{tabular}{lll}
\hline Designation & Lower bound $[\mathrm{Hz}]$ & Upper bound $[\mathrm{Hz}]$ \\
\hline Type 1 & 55 & 100 \\
Type 2 & 101 & 250 \\
Type 3 & 251 & 500 \\
Type 4 & 501 & 750 \\
Type 5 & 751 & 1,000 \\
Type 6 & 1,001 & 2,000 \\
\hline
\end{tabular}

Table 4.5: Frequency bands used to investigate the composition of events contributing to periods of high activity identified by intermittency of wavelet coefficient energy.

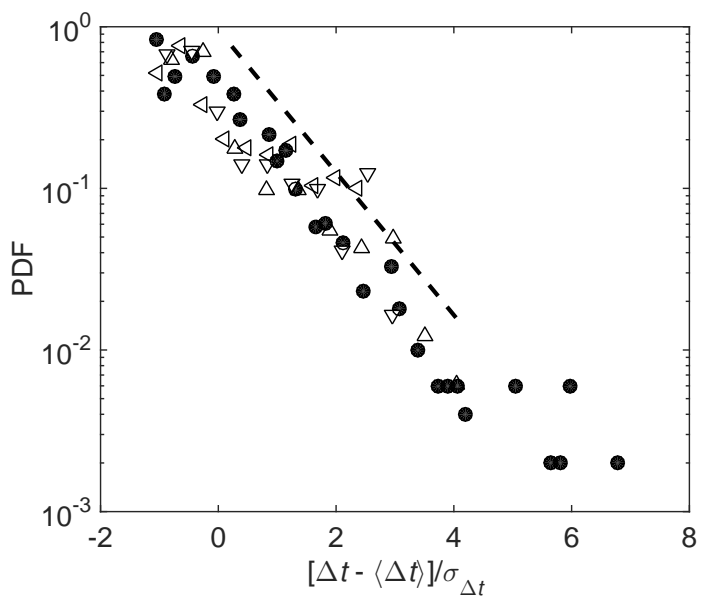

Figure 4.19: Probability density function of time delay for successive events and various flow conditions using higher-order statistics for event detection: $\bullet$, Camussi et al. [17]; $\bigcirc, R e_{\theta}=1,886 ; \triangle, R e_{\theta}=2,546$. Dashed line denotes pure exponential decay.

\subsubsection{Events detected in a sample pressure ensemble}

A typical wall-pressure signal is shown in Fig. 4.20a, along with the calculated timedependent kurtosis of the wavelet coefficients over all scales (as shown in Fig. 4.20b).

Two large-scale events (1.785 $\mathrm{s} \leq t \leq 1.788 \mathrm{~s}$ and $1.793 \mathrm{~s} \leq t \leq 1.801 \mathrm{~s})$ are identified by prolonged kurtosis signatures above the noise threshold. Between these periods of high-activity is a region of low-activity (1.788 $\mathrm{s} \leq t \leq 1.793 \mathrm{~s})$, which exhibits a constant 


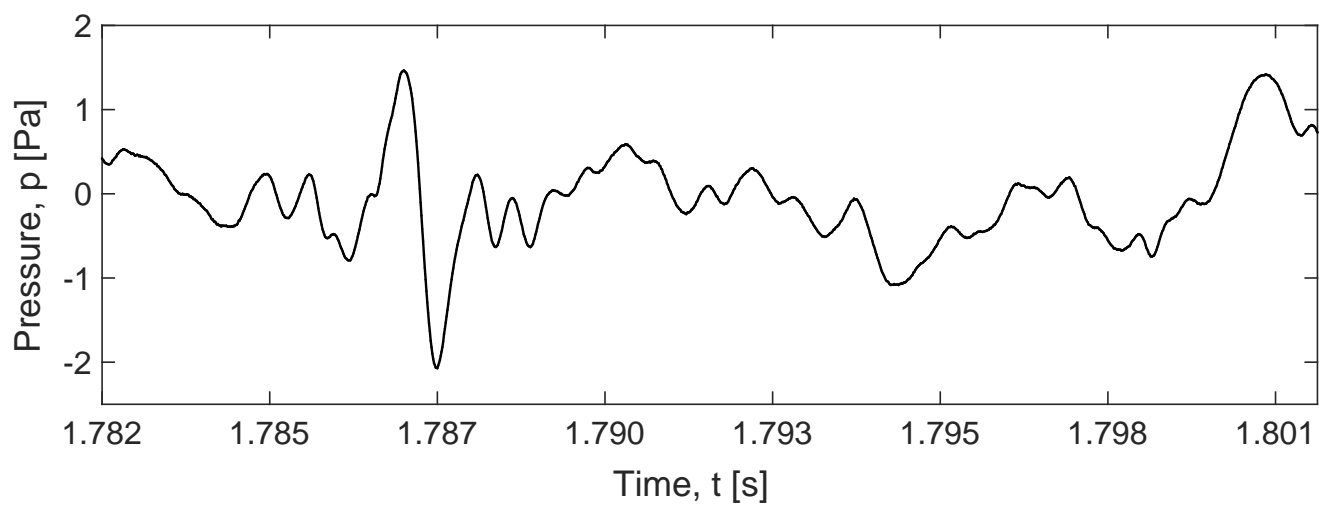

(a)

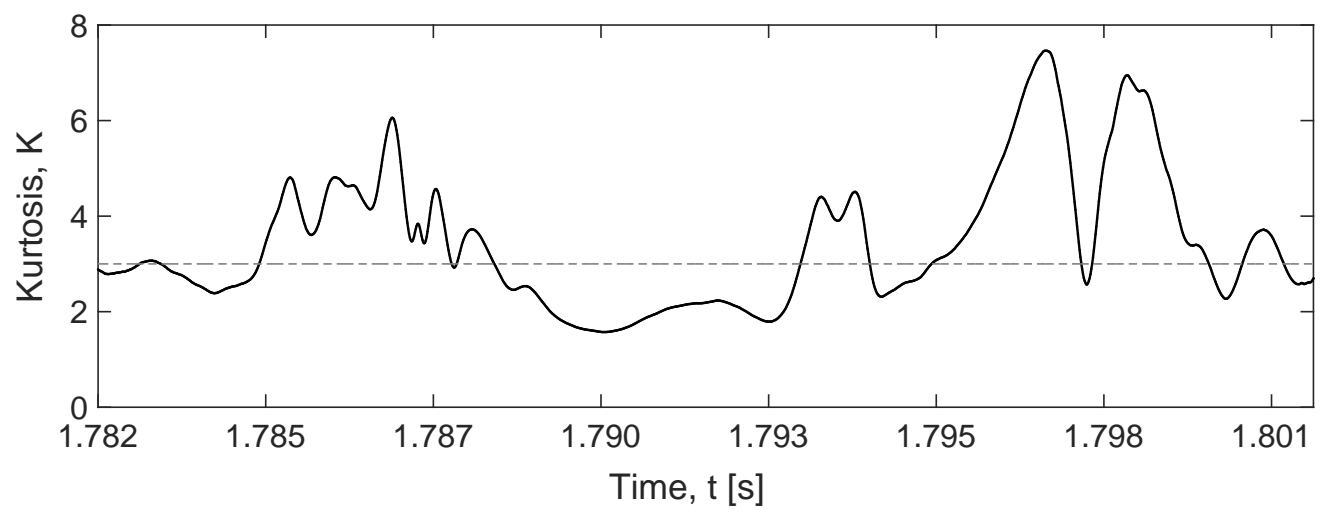

(b)

Figure 4.20: Event detection using the continuous wavelet transform for $\operatorname{Re}_{\theta}=2,546$ : a) sample pressure signal, and b) —, the kurtosis signature based on all scales in the wavelet transformation. = = =, represents the Gaussian noise threshold for event localization.

kurtosis signature below the noise threshold. This alternating behaviour is typical in each of the analyzed time series of pressure, and has similarly been measured using wavelet transforms of wall-pressure fluctuations collected in flight [18]. Within each period of high-activity are large oscillations in pressure, and during each low-activity period a subtle, and sustained, positive pressure signature, with only small oscillations in pressure, is observed.

The multi-scale nature of the high-activity periods is revealed through the kurtosis 
signatures computed for each frequency band. This will be studied, in greater detail, for the first period of high-activity identified in Figure $4.20 \mathrm{~b}(1.785 \mathrm{~s} \leq t \leq 1.788 \mathrm{~s})$. The kurtosis signature, computed using all scales, is magnified for clarity in Figure 4.21a, and compared to the signatures computed for the first five frequency bands in Figure $4.21 \mathrm{~b}$.

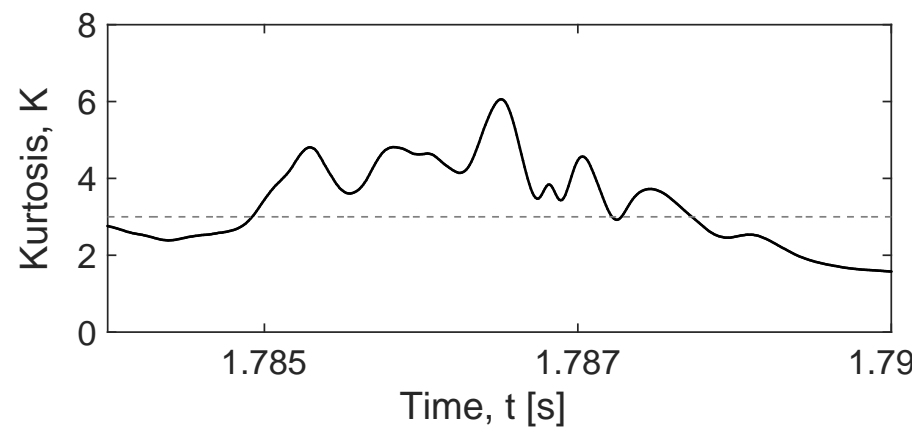

(a)

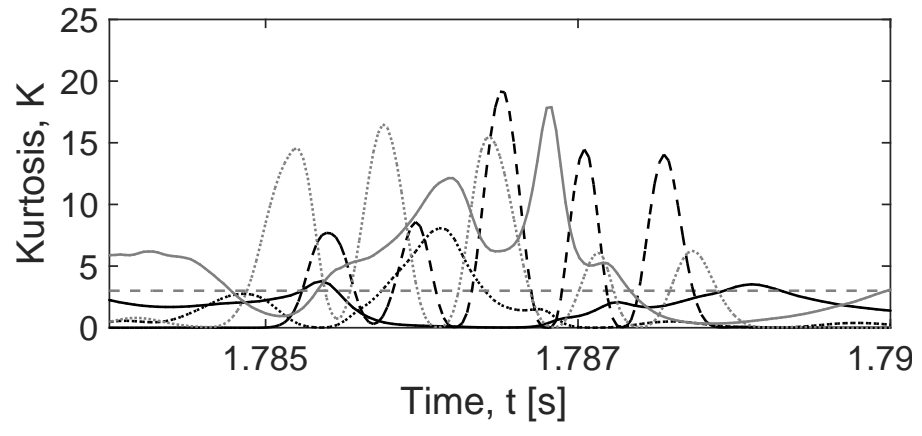

(b)

Figure 4.21: Comarison of kurtosis signature computed using all wavelet scales (a), compared to the signatures computed over each frequency band (b), to illustrate the multi-scale nature of the large-scale events. In (b): —, Type 1; —, Type $2 ; \cdots . .$. , Type $3 ; \cdots .$. , Type $4 ;-\cdot-$, Type 5 .

The time variations in kurtosis, for each frequency band, reveals that large-scale, high-activity events contain a heiracrchy of smaller-scale events. Specifically, the largescale event is spanned by two Type 2 events, which are accompanied by several Type 4 and Type 5 events (Fig. 4.21b). The Type 1 events appear to occur on either side of the smaller-scale structures, which was typical for most periods of high-activity that were 
identified. The majority of large-scale events induce a pair of Type 2 events, and at least two Type 3 events; however, as many as eight Type 3 events were detected in the case of the most persistent events. Other common features, associated with these largescale events, include: as many as 13 consecutive Type 5 events, and brief Type 4 and Type 5 events occuring on the leading and trailing edges of the event. The periods of low-activity occured simultaneously with a single Type 1 event, or Type 2 event, and with no higher frequency events.

Using the Gaussian threshold to extract the start and end of each event, Figure $4.22 \mathrm{a}$ shows how the peaks in the kurtosis signatures relate to distinct fluctuations in wallpressure, which is magnified in Figure 4.22b. This is only shown for Type 2, Type 4, and Type 5 events for clarity.

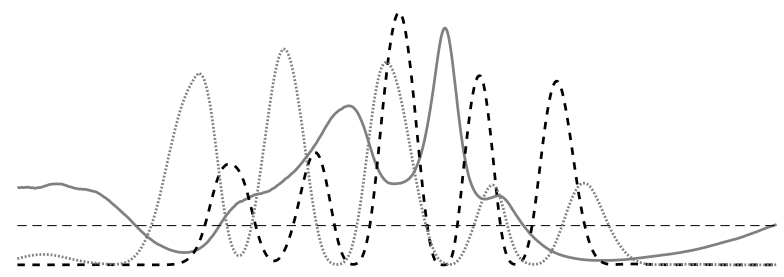

(a)

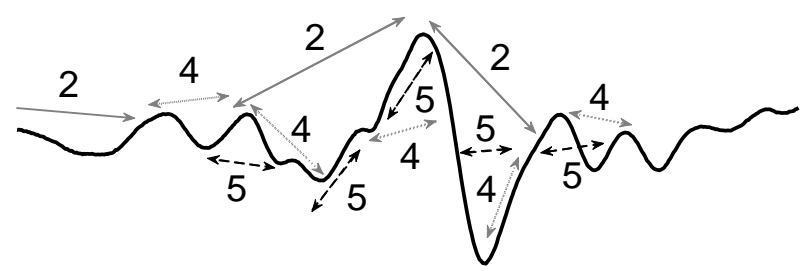

(b)

Figure 4.22: The association of (a) frequency band kurtosis signatures, with (b) features of measured wall-pressure. In (a): —, Type $2 ; \ldots . .$. , Type $4 ;$. . ., Type 5.

Type 2 events span the large positive and negative pressure peak, which appears to be the combined response of several aligned Type 4 and Type 5 events. Type 4 and 
Type 5 events are also associated with the smaller pressure fluctuations on the leading and trailing edges of the large pressure fluctuation.

Many features of the measured pressure signals, and composition of events observed within the large-scale events, are consistent with properties of convecting hairpin vortex packets [19, 69, 112]. To illustrate this, a schematic of a simplified hairpin packet is shown in Figure $4.23 \mathrm{a}$, along with the expected features of the induced wall-pressure signature, as shown in Figure $4.23 \mathrm{~b}$.

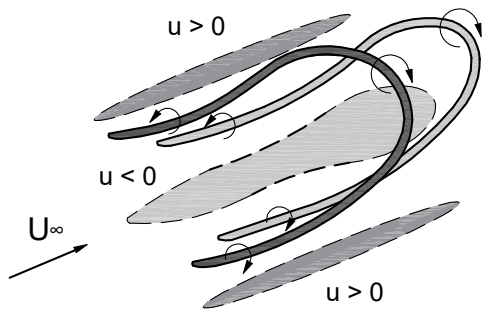

(a)

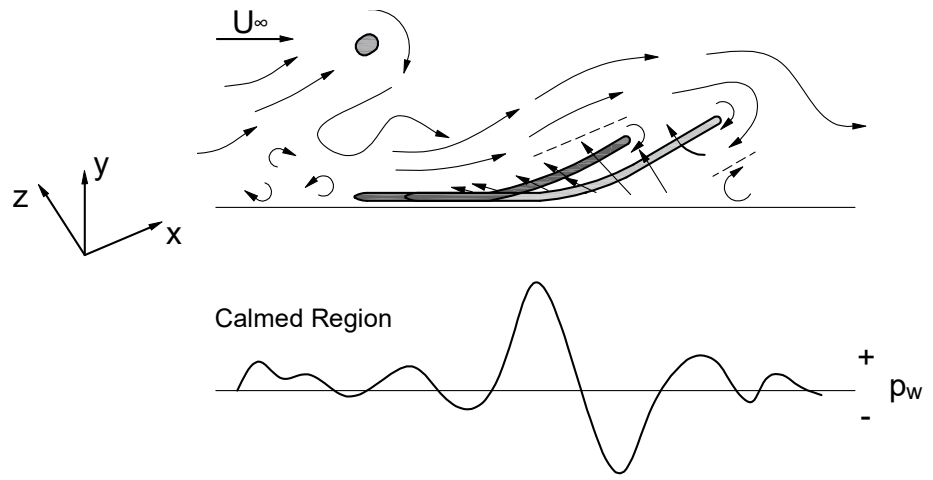

(b)

Figure 4.23: a) Schematic representation of an idealistic two-hairpin packet, and b) expected features of the wall-pressure signature. Dashed line in b) denotes an inclined shear layer.

The counter-rotating streamwise vorticity in the legs of the hairpin (Figure 4.23a create a focused ejection event of low-momentum fluid away from the wall. This ejected fluid forms a low-speed streak between the legs $(u<0)$ and induces strong downwash along the outside of the legs, creating high-speed streaks $(u>0)$ [27]. The signature of the low-speed streak is greater because of the combined induction effects, from the legs and the head, which create a more focused flow [111]. In the case of aligned hairpin vortices, consecutive ejections create a prolonged low-speed streak for the packet. This low-speed streak can remain coherent for a far greater streamwise distance compared to that from an individual hairpin [19,69, 111]. 
The positive pressure peak (Figure 4.23b) arises from the shear layer that forms, as the ejected fluid is opposed by higher momentum fluid. Shear layers may form on both sides of each hairpin, or only at the upstream side of the hairpin packet, depending on the spacing of the vortices within the packet [21]. For the case shown in Figure $4.23 \mathrm{~b}$, it is assumed that the two vortices are positioned along the same shear layer interface.

Negative pressure peaks are generated upstream of the shear layer, due to a largescale sweep event, or downstream of the shear layer, due to the ejection of lowmomentum fluid away from the wall [21, 22, 77]. Negative pressure peaks may also be formed due to the passing of a quasi-streamwise vortex core near the wall [22, 114]. De Ojeda \& Wark [77] measured a streamwise separation of 125 viscous units, between the negative and positive pressure peaks, which is typical of hairpin vortex lengths for $1,520 \leq R e_{\theta} \leq 5,790[19,22]$.

This arrangement of coherent motions creates the alternating positive and negative wall-pressure signature shown in Figure 4.23b. Clearly, the relative amplitude of wallpressure will vary depending on the strength and organization of the hairpin vortices forming the packet. A 'calmed region' may occur in the wake of a hairpin packet, due to induced flow from older hairpins in the outer layer, or entrained non-turbulent fluid between packets [19,77]. In the latter case, the calmed region will be much shorter than that shown in Figure $4.23 \mathrm{~b}$.

Based on a brief literature review, the prolonged regions of elevated kurtosis, associated with a large positive and negative pressure peak, as shown in Figure 4.22b, resembles that of a quasi-streamwise aligned hairpin packet, which induces one large shear layer. The signature of the packet appears to be captured within the Type 2 and Type 3 events, which span the period of elevated kurtosis. Several smaller peaks, with welldefined kurtosis signatures, captured as Type 4, Type 5 and Type 6 events, are nested within, and around, the Type 2 and Type 3 events. This suggests these events represent 
the combined signature from the streamwise alignment of many hairpins.

The large number of Type 4, Type 5 and Type 6 events within the large-scale event, is consistent with the flow visualization study by Adrian et al. [19] who dentified as many as 10 hairpins within a packet. Therefore, these events are thought to represent individual hairpin vortices near the wall. The presence of smaller events on the leading edge of the a high amplitude pressure fluctuation is also consistent with the simulations of Brinkerhoff \& Yaras [27], and experimental measurements by Zhou et al. [112], who showed that strong vorticity in larger hairpins can induce smaller vortices upstream of the head.

\subsubsection{Characteristics of individual events}

To support the physical interpretations of the wall-pressure kurtosis signatures, the events identified in each frequency band will be studied in greater detail by examining the length and convective properties. Kurtosis signatures were tracked between measurements collected over the microphone array, with the time delay between peaks across the microphones used to define the length of the event, and its convection speed.

The PDF of event length, $\mathrm{x}_{\text {event }}^{+}$, as a function of center frequency, is presented in Fig. 4.24 for both flow conditions. It should be noted that Type 6 events were identified in each pressure signal, however, these events often could not be reliably tracked and are therefore, not reported.

Both PDFs show that as the frequency increases the average length of the events decrease in a manner that is well-characterized using a power law function. These functions are shown by the dotted and dashed lines, for $R e_{\theta}=1,886$ and $R e_{\theta}=2,546$, respectively. This fitting would not serve as a physical model for lower frequencies $(f<50 \mathrm{~Hz})$ since the boundary layer limits the maximum size and lifetime of coherent events. There 


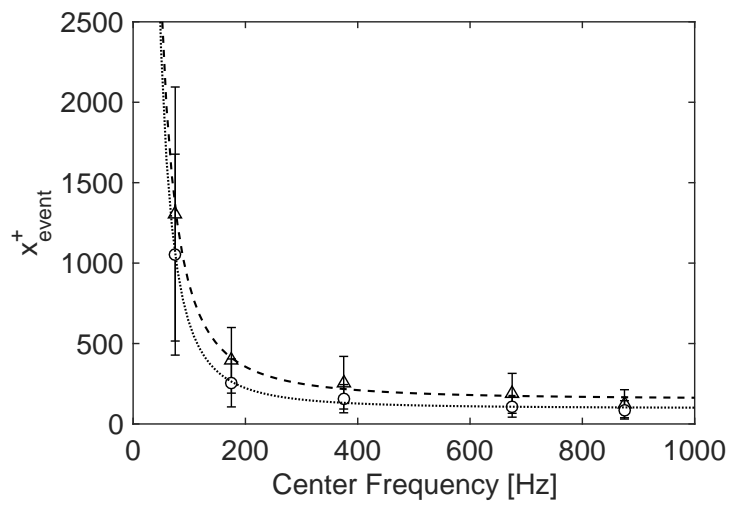

Figure 4.24: Probability density function of event length as a function of frequency: $\bigcirc$, $R e_{\theta}=1,886 ; \triangle, R e_{\theta}=2,546$. Dotted and dashed lines denote power law fittings for $R e_{\theta}=1,886$ and $R e_{\theta}=2,546$, respectively.

appears to be a slight Reynolds number dependence on the size of events, with all events having a shorter average length at $R e_{\theta}=1,886$ compared to $R e_{\theta}=2,546$. Further experiments spanning a larger $R e_{\theta}$ range would be required to properly confirm this finding. This could not be performed in the current facility due to limited available distance for boundary layer development, which limits the conditions in which fully developed TBL conditions can be achieved over the microphone array.

The large variance associated with Type 1 events can be attributed to the lower kurtosis levels in this frequency band. In some instances, the development of a long event could be implied, however, the kurtosis levels over much of the event did not exceed the threshold for detection. To avoid gross underestimation of the event duration, a kurtosis threshold of 1 was used to define the start and end of these events. This shows that the low-frequency events are subtle and persistent, unlike the distinct signatures induced by the higher frequency events. The kurtosis functions of Type 1 events were highly dynamic, making the tracking of kurtosis peaks difficult. In cases where the peak of interest was not a local maximum, the organization of higher frequency events were used to localize the proper peak in the kurtosis signature. These events were always present during periods of low activity, but typically did not span an entire event packet. 
The maximum streamwise coherence detected for these events was $1.9 \delta$, however, the majority did not persist beyond streamwise distances of $0.8 \delta$. All of these characteristics imply that the signature of Type 1 events is representative of intermittent and irrotational flow above the boundary layer [68,73], or entrained valleys of non-turbulent fluid between large-scale motions.

As frequency increases, the variance of extracted event lengths reduces significantly for both flow conditions, indicating a greater level of self-similarity between smaller events. This has been shown to be typical of individual hairpin vortices within a packet [112]. Between the two flow conditions, Type 3 events and Type 4 events had average lengths ranging from $157 \leq x^{+} \leq 204$, and $104 \leq x^{+} \leq 183$, respectively. These lengths are typical of the near-wall hairpins, as identified by Adrian et al. [19], and Ghaemi \& Scarano [22], who approximated these events to have a length of approximately 200 and 160 viscous wall units, respectively. Type 5 events had average lengths on the lower end of estimations from the literature [19, 22], ranging $87 \leq x^{+} \leq 112$, but are believed to also represent smaller hairpin vortices. These events could typically be tracked for $0.5 \delta$, but in some cases up to $1.7 \delta$, similar to that of Type 3 and Type 4 events. This emphasizes the need for high spatial resolution, as these events would not have been detected using the spatial resolution of the rectangular microphone array.

The variation in convection velocity, as a function of frequency, is shown for the conditions of $R e_{\theta}=1,886$ and $R e_{\theta}=2,546$ in Fig. $4.25 \mathrm{a}$ and Fig. 4.25b, respectively. These measurements are compared against measurements from the literature, and predictions using the models proposed by Leclerq \& Bohineust [40] (Eq. 3.28), and Smol'yakov [105] (Eq. 3.27)). The convection velocity of events was derived using adjacent pairs of microphones, with an average spatial separation of $\xi=0.0177 \mathrm{~m}$. Therefore, this value was used to generate the predicted curve with the Leclerq \& Bohineust model. 


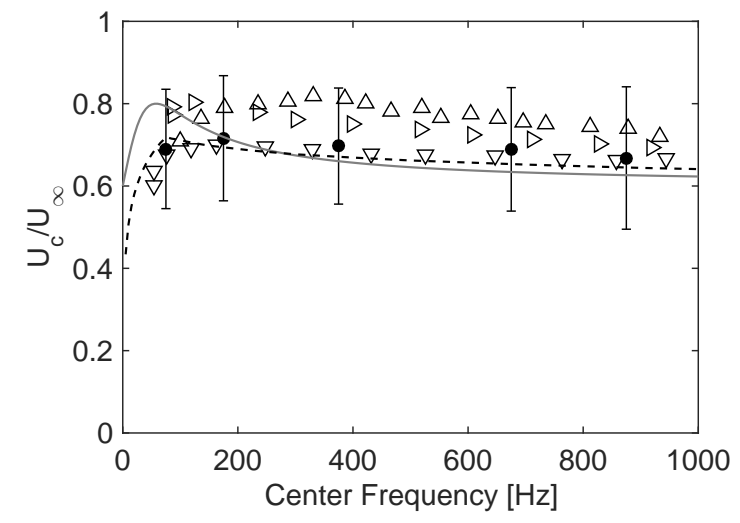

(a)

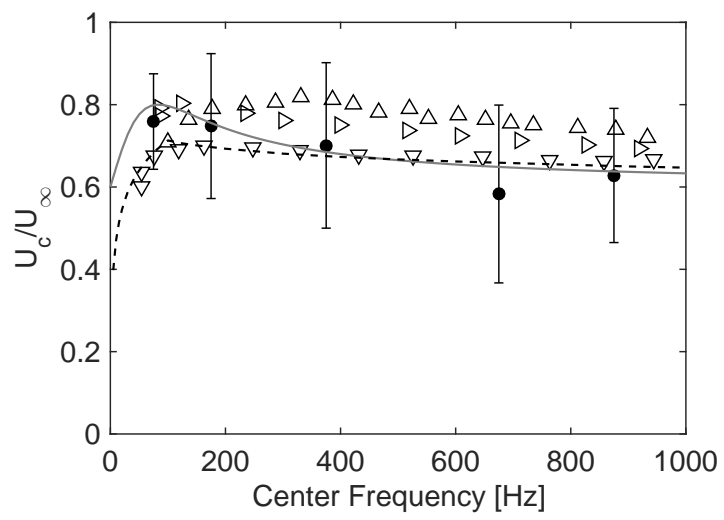

(b)

Figure 4.25: Average convection velocity, as a function of frequency, for events detected using higher-order statistics: a) $R e_{\theta}=1,886$, and b) $R e_{\theta}=2,546 ; \triangle$, Salze et al. [42]; $\nabla$, Farabee \& Casarella [29]; $\triangleright$, Arguillat et al. [3]; - Smol'yakov model [105]; - - ., Leclerq \& Bohineust model [40].

For both flow speeds, the average velocities are in good agreement with the literature and predictions from empirical models. The behaviour of the convection velocity shows a subtle decrease with increasing frequency. The average convection velocities over all events is $0.69 U_{\infty}$ and $0.72 U_{\infty}$ for $R e_{\theta}=1,886$ and $R e_{\theta}=2,546$, respectively, which corroborates the finding of Thomas \& Bull [65] $\left(U_{c}=0.67 U_{\infty}\right)$. Farabee \& Casarella [29] showed that the convection velocity is dependent on relative separation between microphones. The spacing is not explicitly reported for the measurements by Salze et al.442] and Arguillat et al. [3], which could explain the slightly higher values in their studies compared to the current measurements.

Adrian et al. [19] showed that the convection speed of packets is highly dependent on its age, size, and relative position in the boundary layer. This is captured in the current measurements, which show large variance at all frequencies. The large variance is also due to evolving patterns in the kurtosis signatures, as the events interact, and change size and strength.

The decreasing convection velocity with increasing frequency agrees well with the 
notion that low frequency events are generally larger and located further away from the wall, where they convect with a higher velocity. Unfortunately, this analysis does not enable one to locate the position of events in the wall-normal direction. However, the approach from Bull [9], of superimposing the convection speed on the mean velocity profile, is used again to approximate the wall-normal position of Type 2 and Type 5 events. At $R e_{\theta}=1,886$, this approach suggests that Type 2 events originate near $y^{+}=100$, but extend as high as $y^{+}=322$, and as low as $y^{+}=33$. Type 5 events are estimated to originate near $y^{+}=65$, and extend as high, and as low as $y^{+}=268$ and $y^{+}=22$, respectively. This is in good agreement with the findings of Chacin \& Cantwell [57], who showed that, at low Reynolds numbers, the large-scale motions can extend over much of the boundary layer and influence the motions near the wall. The overlap between large-scale Type 2 events, and small-scale Type 5 events, also agrees with the study by Adrian et al. [19] who located several smaller hairpins within larger hairpins throughout the log-region of the boundary layer. This also suggests that the low-frequency wallpressure energy is primarily generated within, and below, the logarithmic region of the boundary layer where the large, low-speed streaks are concentrated [19, 115], and supports the findings from wall-pressure-velocity cross-spectrum measurements.

\subsubsection{Characteristics of event packets}

An analysis is also performed to extract information regarding the large-scale motions, identified within the kurtosis signatures computed using all scales, to support inferences regarding their association with packets of small-scale structures. The packets are characterized by the start and end of the Type 4 or Type 5 events on the leading and trailing edges of a group of Type 2 events. From this, the length of each packet and the relative packet spacing can be determined. The convection speed of the packets were estimated using the average convection velocities measured for Type 1, Type 2 and Type 3 events 
occurring within the prolonged period of high-activity. These events are selected as they are presumed to convect the higher frequency events downstream.

Characteristics of the hairpin packets, detected in the five wall-pressure ensembles, for each speed, are listed in Table 4.6, and the PDF of the average number of event types in each packet is shown in Fig. 4.26 for both flow conditions. Measured properties of the event packets, on which the values in Table 4.6 are based, can be found in Appendix A. For each packet the viscous-normalized length $\left(x^{+}=T \cdot U_{c} u_{\tau} / v\right.$, where $T$ denotes the time duration of the packet), average convection velocity $\left(U_{c}\right)$ and number of events in each frequency band were recorded. $\sigma$ denotes the standard deviation of the subscripted quantity.

\begin{tabular}{lllllllllll}
\hline$R e_{\theta}$ & $x^{+}$ & $\sigma_{x^{+}}$ & $U_{c}$ & $\sigma_{U_{c}}$ & Type 1 & Type 2 & Type 3 & Type 4 & Type 5 & Type 6 \\
\hline 1,886 & 1,011 & 694 & 0.71 & 0.09 & 1.0 & 2.1 & 3.0 & 3.1 & 2.7 & 1.9 \\
2,546 & 1,939 & 962 & 0.73 & 0.07 & 1.0 & 3.0 & 4.0 & 4.7 & 5.0 & 4.6 \\
\hline
\end{tabular}

Table 4.6: Properties of event packets detected using intermittency of all wavelet scales between $55 \mathrm{~Hz}$ and 2,000 $\mathrm{Hz}$.

For both flow conditions, the packets convected at an average speed of approximately $0.7 U_{\infty}$, which is in good agreement with space-time correlation measurements reported in section 4.4.1 and results from the literature [7, 29, 40, 65, 69, 70, 77।. Further, the range of convection velocities measured within packets $\left(0.53 \leq U_{c} / U_{\infty} \leq 0.93\right)$, agrees with the range of speeds measured within hairpin packets by Meinhart \& Adrian [69] $\left(0.5 U_{\infty}\right.$ to $\left.1.0 U_{\infty}\right)$. On average, the packets at $R e_{\theta}=1,886$ were approximately $50 \%$ shorter in length, compared to the packets at $R e_{\theta}=2,546$. This likely explains the fewer number of events in all categories except Type 1 events, which appear to be insensitive to the size, and number of events that occur within each packet. This supports the idea 
that these events are related to outer-layer intermittency created between the packets.

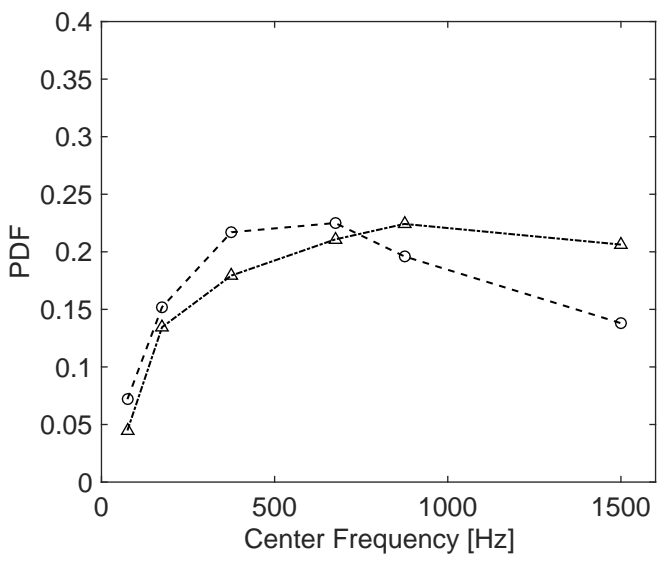

Figure 4.26: Probability density function of event packet composition: ${ }^{-} \odot-, \operatorname{Re}_{\theta}=1,886$; $-\triangle, e_{\theta}=2,546$.

The probability of occurrence of an event in each frequency band is computed, based on the values provided in Table 4.6, to compare the multi-scale compositions of packets at both speeds. The resulting PDFs are shown in Fig. 4.26. Fig. 4.26 shows that the relative composition of events shifts to higher frequencies with increasing $R e_{\theta}$. In the packets at $R e_{\theta}=1,886$, Type 3 and Type 4 events are most prominent, whereas at $R e_{\theta}=2,546$, Type 5 events occur most frequently. This is explained by the change in turbulence structure that occurs at low $R e_{\theta}$, from large individual hairpin vortices, to more numerous smaller vortices, with increasing $R e_{\theta}$ [57, 61, 109]. This measured shift in composition supports the hypothesis that the signatures of Type 3, Type 4 and Type 5 events are likely that of individual hairpin vortices, while Type 2 events are most likely associated with large hairpin vortices or the signature of a hairpin packet.

The coherence of events and packets at $\operatorname{Re}_{\theta}=1,886$ were generally more difficult to track, compared to $\operatorname{Re}_{\theta}=2,546$. This could be due to the fact that the hairpin vortices, that form at lower Reynolds numbers, are weaker and cannot sustain their coherence as long, or do not generate offspring vortices as efficiently. It was shown by Zhou et al. 
[112] that hairpin vortices must be of sufficient strength to contribute to the autogeneration of newer vortices. As Reynolds number increases the combined effects of increasing hairpin strength and a greater number of vortices, contributes to more efficient autogeneration. This is supported by the greater number of high-frequency events at $R e_{\theta}=2,546$, as shown in Fig. 4.26 .

The evolution of detected large-scale motions generally followed two trends: one where the packet grew in length with an increased average convection velocity, or decreased in length with decreasing average convection velocity. Adrian et al. [19] suggests that the growth of a packet and increase in convection speed is characteristic of more mature hairpin packets, due to the outward growth of the primary hairpins from the wall, which become entrained with higher momentum fluid. The decrease in length and convection velocity is thought to represent decaying packets due to interaction with other events, or viscous diffusion.

The maximum streamwise coherence of event packets was measured to be $1.9 \delta(64.6$ $\mathrm{mm})$ and $4 \delta(140 \mathrm{~mm})$ for $R e_{\theta}=1,886$ and $R e_{\theta}=2,546$, respectively. In the case of $R e_{\theta}=2,546$, this happened to coincide with the largest spacing permitted between microphones using the central microphone as the reference. Therefore, the total extent of these events is estimated using the Fourier-based cross-spectra for both speeds ( $\hat{L}_{x}=55 \mathrm{~mm}$ and $\hat{L}_{x}=82 \mathrm{~mm}$ for $R e_{\theta}=1,886$ and $R e_{\theta}=2,546$, respectively). The coherence length distribution shown in Fig. $4.11 \mathrm{~b}$ is derived from an exponential fitting to the cross-spectrum amplitudes, between each microphone in the array, and a reference microphone, at each frequency. Therefore, the total extent of the packets are estimated by three decay constants, or three times the maximum coherence lengths reported in Fig. $4.11 \mathrm{~b}$. Based on this, it is estimated that maximum spatial coherence of the packet is $4.8 \delta(164 \mathrm{~mm})$ and $7.1 \delta(245 \mathrm{~mm})$ at $R e_{\theta}=1,886$ and $R e_{\theta}=2,546$, respectively. 
The coherence lengths are in agreement with reported values for long, lowmomentum streaks, expected for hairpin packets [20, 82, 110, 111]. The maximum lengths of the packets, from the current measurements, are more appropriately represented using Fourier-based coherence lengths at $197 \mathrm{~Hz}$ and $165 \mathrm{~Hz}$ for $R e_{\theta}=1,886$ and $R e_{\theta}=2,546$, respectively. Both frequencies fall within the expected frequency range for Type 2 events and validates the approach used for tracking event packets using Type 2 events. However, the measurements do not explain the source of maximum coherence in wall-pressure signatures. Hutchins \& Marusic [110] suggest that the true spatial extent of large-scale motions may be masked from single-point measurements by spanwise meandering of the structure. This is believed to be the most likely explanation of the discrepancies between measured hairpin packet coherence, and the maximum coherence length from the Fourier-based coherence length analysis.

\subsubsection{Comments on the use of higher-order statistics and wavelet transforms for event detection}

This section discusses the advantages and limitations of using intermittency of wavelet coefficients for event localization within wall-pressure measurements.

Intermittency provides a quantitative way of localizing events within a wavelet transform, and enables periods of high-activity to be examined on a multi-scale level. The use of a high resolution microphone array, enabled highly dynamic pressure signatures, from high-frequency structures, to be tracked.

This technique identified a number of events that cannot be explained using the concept of a single hairpin packet. For example, intermittent, large, Type 2 and 
Type 3 events were observed with very few high-frequency events nearby. These signatures are believed to be representative of randomly scattered, individual hairpin vortices, as measured in the PIV study by Zhou et al. [112]. Further, some periods of extended, elevated kurtosis would temporarily fall below the threshold for detection, or divide into two or more smaller events. This is thought to be the result of varying convection speeds of the coherent structures, contributions from multiple generations of structures at different positions within the boundary layer, and spanwise meandering of the large-scale motions as they convect downstream [19, 27, 110, 111]. As these structures meander, adjacent microphones may capture a different phenomena associated with the same structure (i.e.: an ejection over one microphone, and a high-speed streak over another microphone). This shows a limitation of using a scalar quantity for event detection, as it does not enable one to determine the direction of motion or rotation. Spanwise meandering may also contribute to variations in number of events and lengths of packets, and/or the perceived disappearance, or break-up of packets between microphones.

The discretization of the wavelet transform into frequency bands may introduce bias in the number of events detected within a packet. For example, if the wavelet transform possesses a region of elevated energy that spans two frequency bands, this is considered as two events, having nearly equal durations. The use of a Gaussian threshold may have also resulted in slight underestimation of the spatial coherence and/or length of individual events, and event packets. In some cases, the kurtosis signature was retained between microphones, but the values had fallen below the detection threshold. This is believed to be the result of the maturation of coherent structures, which move away from the wall and become diffused due to viscous effects and interactions with other coherent motions.

Despite the range of physical processes contributing to the wall-pressure signal, 
characteristics of the detected events show good consistency with the literature in regards to event duration, organization, and convection velocity. This suggests that detection of coherent structures in a TBL may not be overly sensitive to the exact arrangement of hairpin vortices, or the effects of spanwise meandering are minimal at the flow speeds considered.

While a microphone array cannot outright replace other instruments for turbulence studies, it has shown good potential to serve as a complimentary tool for studying properties of coherent structures, and simultaneously establishing a better understanding of their relationships with the wall-pressure field. In combination with turbulence control techniques, to manipulate the alignment of the hairpin vortices, wall-pressure transducers may be used to establish more definitive relationships between wall-pressure fluctuations and physical features of individual hairpin vortices and aligned vortex packets. 


\section{Chapter 5}

\section{Conclusions and Recommendations}

\subsection{Summary of Measurements}

This thesis outlined the experimental studies conducted to characterize the spatial coherence of turbulence-induced wall-pressure fluctuations. Results from a preliminary study are presented in which the effects of two flush-mounted microphone cap configurations, on the measured wall-pressure spectra, are assessed. Results from that study justified the acquisition of a hotwire anemometry system, and the need to design an adjustable ceiling to control the streamwise pressure gradient in the test section.

Traditional statistical descriptors of wall-pressure fluctuations, including the power spectral density, cross-spectrum, and time-averaged cross-correlations, were used to deduce the high-level structure of coherent motions within the TBL. The predictive capabilities of a selection of empirical models, for each of these descriptors, were assessed using experimental measurements. More advanced analyses were conducted, using wavelet transformations, to extract detailed information regarding the organization of coherent, turbulent structures that contributed to measured wall-pressure fluctuations. A cross-correlation analysis was conducted using energy in the wavelet coefficients to investigate the convective nature of large-scale motions. The contribution of large-scale 
events, to the Fourier-based cross-spectrum, was explored using a conditional averaging cross-spectrum analysis. Finally, a novel, statistical post-processing technique was developed and presented, for extracting coherent motions using intermittency of energy in the wavelet coefficients. This was used to study the large-scale events, identified in the cross-correlation analysis, and their multi-scale nature using the intermittency of energy within frequency bands.

\subsection{Conclusions}

The initial phase of this research showed that a pinhole cap was required to properly capture the high-frequency wall-pressure energy. The grid cap resulted in attenuation of energy in the overlap frequency range and its slotted surface induced resonance at much lower frequencies than the pinhole cap. The high-frequency energy in the spectrum decayed faster than expected, which, through spectrum normalization, was attributed to an under-developed boundary layer profile.

Fully-developed conditions were achieved at two flow speeds, $U_{\infty}=7.6 \mathrm{~ms}^{-1}$ $\left(R e_{\theta}=1,886\right)$ and $U_{\infty}=10.4 \mathrm{~ms}^{-1}\left(R e_{\theta}=2,546\right)$, following the installation of an adjustable ceiling, to control the streamwise pressure gradient, and an extended floor to allow greater boundary layer development. These two flow speeds were considered for more extensive studies of the wall-pressure fluctuations. Measured wall-pressure spectra showed higher energy, within and above the overlap frequency range, while inner layer-normalized spectra collapsed onto measurements from the literature at similar flow speeds. Goody's empirical model was found to be most accurate for modeling the single-point wall-pressure spectrum for the Reynolds numbers analyzed. The models developed based on measurements at much higher flow speeds cannot be extrapolated to lower speeds. 
The double-exponential model for the streamwise decay in cross-correlations suggested that turbulent structures, contributing to the wall-pressure energy, can be coarsely divided into large-scale and low-frequency, or small-scale and high-frequency. The large-scale motions decay up to ten times slower, but do not directly contribute much energy to the single-point spectrum (as low as $12 \%$ ).

Cross-spectrum analysis of wall-pressure and velocity fluctuations were in agreement with the literature and confirmed that near-wall velocity fluctuations are influenced by large-scale motions. The low-frequency and high-frequency peaks in the coherence function, for velocity fluctuations in the buffer layer, were associated with smaller hairpin packets, located very near the wall, that occur within larger packets. The effects from small-scale structures, identified in the buffer layer, have limited extent in the logarithmic region of the boundary layer, which explains the decrease in highfrequency energy, with increasing distance from the wall.

The convection velocity increased with increasing spatial separation, and ranged from $U_{c}=0.67 U_{\infty}$ to $U_{c}=0.82 U_{\infty}$. The convection velocity was used to approximately locate large-scale and small-scale structures within the outer-wake region and logarithmic region, respectively. Measurements confirmed that large-scale motions contribute the majority of energy, near the peak in the single-point frequency spectrum, while events contributing to the overlap range decay within traveling $10 \delta^{\star}$ downstream.

Cross-spectrum measurements collapsed when plotted against normalized frequency, $\omega \delta / u_{\tau}$, indicating a self-similar nature of the boundary layer and its convected pressure signature. Low-frequency pressure signatures, associated with peak energy in the power spectrum, are retained for up to $4 \delta$ in the streamwise direction. The model of Leclerq \& Bohineust [40] accurately captures the peak in the cross-spectrum; however, the reported empirical constants could not be used to predict the cross-spectrum at 
higher frequencies. This was a result of the weaker normalization compared to the Corcos similarity scaling. The Corcos model showed to be a good candidate for predicting the cross-spectrum amplitudes at frequencies above $\omega \delta / u_{\tau}=50$. The calibrated decay constants for the Corcos model exhibit a Reynolds number dependency, showing more gradual decay rates with increasing $R e_{\theta}$. This was confirmed using a coherence length analysis, based on the Corcos model, which revealed that high-frequency fluctuations retain their coherence for a greater streamwise distance as $R e_{\theta}$ increases.

The wavelet cross-correlation function revealed that small-scale motions occur within intermittent large-scale motions. Conditional cross-spectrum analyses showed that the majority of events contributing to the Fourier-based cross-spectrum energy are concentrated within intermittent low-frequency, large-scale motions. This intimate relationship, between high-frequency and low-frequency events, suggests that the largescale motions represent the signature of convecting packets of smaller coherent events.

The use of higher-order statistics for event detection enabled greater detail to be extracted from wavelet transformations of wall-pressure measurements. The kurtosis signature, computed over all scales, revealed intermittent, but extended, periods of elevated activity. Discretizing the wavelet transform into frequency bands revealed the underlying organization of events within these packets. This analysis associated pressure fluctuations occurring at $55 \mathrm{~Hz} \leq f \leq 100 \mathrm{~Hz}$ with irrotational motions/entrained fluid between large-scale structures. Events occurring between $101 \mathrm{~Hz} \leq f \leq$ $250 \mathrm{~Hz}$ represent large-scale vortices, or the aggregate signature of consecutive smaller events, arranged in a quasi-streamwise packet. These packets consist of events between $250 \mathrm{~Hz} \leq f \leq 1,000 \mathrm{~Hz}$. The average length of these events ranged from $x^{+}=87$ to $x^{+}=204$, which is consistent with reported measurements of hairpin vortices in the literature.

The composition of events within hairpin packets shifted towards higher-frequency 
events with increasing $R e_{\theta}$, which is consistent with the physics of low Reynolds number turbulent boundary layers. Spanwise meandering of the large-scale motions, and limited spatial coverage of the microphone array, appears to mask the full streamwise extent of the hairpin vortex packets. That said, other important characteristics, such as event duration, organization, and convection velocity, were reliably measured using two arrays spanning $4 \delta$ in the streamwise direction. This analysis shows good potential for use in more detailed studies on the physics of turbulence and its relationship with wall-pressure excitation.

\subsection{Research Contributions}

The research contributions presented in this thesis are summarized in this section.

1. (Chapter 2) Design, installation, and commissioning of a removable, flexible ceiling to enable manipulation of the streamwise pressure gradient over the test section.

2. (Chapter 2) The successful implementation of a rotating instrumented test panel for standard 1/4 inch microphones, based on a design proposed by Robin et al. [49]. The panel consists of six linear arrays of holes, offset by $60^{\circ}$, that enables time-averaged characterization of the wall-pressure spatial decay with a resolution down to $1.6 \mathrm{~mm}$. This is a significant improvement over the array in the first test panel, which had a maximum spatial resolution of $25.4 \mathrm{~mm}$, and exceeds the highest reported spatial resolution in the literature for standard 1/4 inch microphone arrays ( $2 \mathrm{~mm}$ resolution reported by Robin et al. [49]).

3. (Chapter 2) Design and assembly of a hotwire probe traverse system for velocity measurements over the test panel. The design consists of a three linear, optical 
stages with a resolution of $1 \mu \mathrm{m}$ in the wall-normal direction and $1 \mathrm{~mm}$ in the streamwise and spanwise directions.

4. (Chapter 3 \& Chapter 4) Development of a novel processing technique for extracting coherent turbulent events and event packets using point measurements of wall-pressure. The analysis developed in this research uses the intermittency of energy in the wavelet coefficients for event localization. This varies from other studies which detect events using the relative amplitude of correlated energy in the wavelet coefficients, between pairs of microphones. Correlation amplitude detection approaches result in low detection rates for small scale events with low relative energy, and limits the scope of those investigations to large-scale events.

In the new approach, the kurtosis of the energy in wavelet coefficients, from measured wall-pressure signals, is computed over all scales to identify periods with high activity. The wavelet coefficients are also sub-divided into frequency bands to study the multi-scale properties of these high-activity periods. The kurtosis of a Gaussian distribution is used to represent the threshold of noise for event detection. Events detected in the first microphone of the array can be tracked in the kurtosis signatures computed for wall-pressure signals measured at each microphone in the array. The time delay between adjacent microphones is then used to define the convection velocity and length of each detected event.

The kurtosis signature, computed for all scales, can be used to localize large-scale motions, thought to be representative of the signature of quasi-streamwise hairpin packets. This approach was successfully applied to associate wall-pressure signatures with coherent turbulent sturctures, study the properties of hairpin vortices and vortex packets, and capture the Reynolds number dependence of the organization of hairpin vortices within the packets. 
Parts of this thesis has been published as:

1. Van Blitterswyk J., Rocha J., Prediction and Measurement of Flow-Induced Wall Pressure Fluctuations at Low Mach Numbers, J. Canadian Acoustics, 42: 3-14, 2014.

2. Van Blitterswyk J., Rocha J., Prediction and Measurement of Turbulent Boundary Layer Wall-Pressure Fluctuations on the Surface of a Single Panel at Low Mach Numbers, Canadian Acoustical Association, Acoustics Week in Canada, Winnipeg, 8-10 October, 42:18-19,2014.

3. Van Blitterswyk J., Rocha J., Wall-Pressure Coherence Beneath a Low Reynolds Number Turbulent Boundary Layer, 24th International Congress of Theoretical and Applied Mechanics, Montreal, 21-26 August, 2016, PO.FM14-1.19.182.

Submitted refereed publications include:

1. Van Blitterswyk J., Rocha J., An Experimental Study of the Wall-Pressure Fluctuations Beneath Low Reynolds Number Turbulent Boundary Layers, Submitted to: J. Sound Vib.

2. Van Blitterswyk J., Rocha J., Detection of Coherent Turbulent Structures Using Wall-Pressure Fluctuations, Submitted to: Phys. Fluids.

\subsection{Recommendations for Future Work}

Although the results of this study are in good agreement with measurements from the literature, a number of areas for improvement have been identified, with regard to the experimental setup and data analysis procedures. 
1. While the adjustable ceiling effectively reduced the streamwise pressure gradient, the effects from boundary layer growth on the side walls of the test section could not be fully compensated with the limited stroke of the ceiling. Further studies can be considered, in which the side walls are covered with a hard surface, to minimize boundary layer growth/separation. The double partition will also serve to further attenuate exterior noise effects within the test section.

2. The experimental wall-pressure spectra indicate that the effects of reduced mean velocity, and Reynolds stress in the buffer layer at $R e_{\theta}=2,546$, are contained to the high-frequency range of the spectrum. The effects on other statistical descriptors, and the organization/production of coherent events are not immediately apparent. Therefore, the cause of this deficiency could be determined in order to better scrutinize its effects.

3. The hotwire traverse system can be automated to reduce testing time and expand the measurement domain. The concatenated design of the wall-normal traverse stages was sufficient for the current flow speeds; however, the system could be improved to reduce probe vibration at higher speeds.

4. Measurements of the cross-spectrum and cross-correlation required repositioning of the microphones, and/or panel rotation between measurements. Crosscorrelation measurements show a slight offset in peak values between different microphone configurations. While not expected to be a major source of error, the sensitivity of these measurements to microphone flushness can be further investigated for improved accuracy.

5. The higher-order statistics approach, to event detection, could be applied to measurements having greater spatial coverage, characterize the streamwise extent of 
large-scale motions. Measurements using spanwise-oriented microphones may also be used to study the spanwise extent of the large-scale motions.

6. The use of frequency bands, for event detection, could be re-evaluated/refined to reduce bias in detection rates due to events spanning neighbouring frequency bands.

7. In this work, events were extracted based on pressure ensembles having 6,144 data points. The ensembles were kept short to reduce the computation time required to obtain the wavelet transformation. The length and number of ensembles could be extended in future work to develop a larger statistical basis. Extraction of event duration, and peak location, could also be automated to reduce processing time.

8. The magnitude of kurtosis was not recorded when localizing events in the wavelet transformations. However, some events showed large changes in the peak kurtosis value as they convected downstream. This may serve as an additional metric for extracting information about the detected events, such as the state/energy of the coherent structure.

9. Findings from the event detection analyses could be complimented with velocity measurements over the panel using a hotwire sensor. The acquisition of a twoaxis hotwire probe would be useful for more accurate characterization of coherent turbulent events, and further studies surrounding their relationship with wallpressure.

10. The statistical event detection analysis presented in this work shows strong potential for studying coherent structures in a turbulent boundary layer. However, 
in naturally developing boundary layers, it is difficult to identify the specific phenomena being detected from the wall-pressure features. This is especially difficult for the small-scale coherent structures, due to the unknown alignment of said structures over the microphone array. Future testing might include turbulence control techniques, such as riblets, to ensure alignment of the hairpin vortices. This would enable one to more definitively correlate wall-pressure measurements with physical features of individual hairpin vortices and aligned vortex packets.

11. Events detected using wavelet coefficient intermittency may be validated with PIV measurements, or numerical simulations. 


\section{Bibliography}

[1] Wilby J.F. Aircraft interior noise. J. Sound Vib., 190:545-564, 1996.

[2] Fahy F. and Walker J. Advanced Applications in Acoustics, Noise and Vibration. Spon Press, London, 2004.

[3] Arguillat B., Ricot D., Bailly C., and Robert G. Measured wavenumber: Frequency spectrum associated with acoustic and aerodynamic wall pressure fluctuations. Phys. Fluids A-Fluid, 128:1647-1655, 2010.

[4] Bull M.K. Wall-pressure fluctuations beneath turbulent boundary layers: some reflections on forty years of research. J. Sound Vib., 190:299-315, 1996.

[5] Mellert V., Baumann I., Freese N., and Weber R. Impact of sound and vibration on health, travel comfort and performance of flight attendants and pilots. Aerosp. Sci. Technol., 12:18-25, 2008.

[6] Camussi R. Noise Sources in Turbulent Shear Flows: Fundamentals and Applications. Springer, Vienna, 2013.

[7] Willmarth W.W and Wooldridge C.E. Measurements of the fluctuating pressure at the wall beneath a thick turbulent boundary layer. J. Fluid Mech., 14:187-210, 1962. 
[8] Corcos G.M. The structure of the turbulent pressure field in boundary-layer flows. J. Fluid Mech., 18:353-378, 1964.

[9] Bull M.K. Wall-pressure fluctuations associated with subsonic turbulent boundary layer flow. J. Fluid Mech., 28:719-754, 1967.

[10] Corcos G.M. Resolution of pressure in turbulence. J. Acoust. Soc. Am., 35:192-199, 1963.

[11] Palumbo D. Determining correlation and coherence lengths in turbulent boundary layer flight data. J. Sound Vib., 331:3721-3737, 2012.

[12] Farge M. Wavelet transforms and their application to turbulence. Annu. Rev. Fluid Mech., 24:395-457, 1992.

[13] Camussi R. and Guj G. Orthonormal wavelet decomposition of turbulent flows: intermittency and coherent structures. J. Fluid Mech., 348:177-199, 1997.

[14] Ruppert-Felsot J., Farge M., and Petitjeans P. Wavelet tools to study intermittency: application to vortex bursting. J. Fluid Mech., 636:427-453, 2009.

[15] Yang S. and Nan J. Wavelet analysis to detect multi-scale coherent eddy structures and intermittency in turbulent boundary layers. Eight International Conference on Fuzzy Systems and Knowledge Discover (FSKD), 2, 2011.

[16] Li H. Identification of coherent structure in turbulent shear flow with wavelet correlation analysis. J. Fluids Eng., 120:778-785, 1998.

[17] Camussi R., Robert G., and Jacob M.C. Cross-wavelet analysis of wall pressure fluctuations beneath incompressible turbulent boundary layers. J. Fluid Mech., 617:11-30, 2008. 
[18] Palumbo D. Deriving lifetime maps of coherent structures in the turbulent boundary layer. AIAA J., 46:810-823, 2008.

[19] Adrian R.J., Meinhart C.D., and Tomkins C.D. Vortex organization in the outer region of the turbulent boundary layer. J. Fluid Mech., 422:1-54, 2000.

[20] Ganapathisubramani B., Longmire E.K., and Marusic I. Characteristics of vortex packets in turbulent boundary layers. J. Fluid Mech., 478:35-46, 2003.

[21] Ghaemi S., Ragni D., and Scarano F. PIV-based pressure fluctuations in the turbulent boundary layer. Exp. Fluids, 53:1823-1840, 2012.

[22] Ghaemi S. and Scarano F. Turbulent structure of high-amplitude pressure peaks within the turbulent boundary layer. J. Fluid Mech., 735:381-426, 2013.

[23] Pope S.B. Turbulent Flows, 2nd Ed. Cambridge University Press, New York, 2000.

[24] Robinson S.K. Coherent motions in the turbulent boundary layer. Annu. Rev. Fluid Mech., 23:601-639, 1991.

[25] Smith C.R. and Metzler S.P. The characteristics of low-speed streaks in the near wall region of a turbulent boundary layer. J. Fluid Mech., 129:27-54, 1983.

[26] Head M.R. and Bandyopadhyay P.R. New aspects of turbulent boundary-layer structure. J. Fluid Mech., 107:297-338, 1981.

[27] Brinkerhoff J.R. and Yaras M.I. Numerical investigation of the generation and growth of coherent flow structures in a triggered turbulent spot. J. Fluid Mech., 759:257-294, 2014.

[28] Keith W.L., Hurdis D.A., and Abraham B.M. A comparison of turbulent boundary layer wall-pressure spectra. J. Fluids Eng., 114:338-347, 1992. 
[29] Farabee T.M. and Casarella M.J. Spectral features of wall pressure fluctuations beneath turbulent boundary layers. Phys. Fluids A-Fluid, 3:2410-2420, 1991.

[30] Rackl R. and Weston A. Modeling of turbulent boundary layer surface pressure fluctuation auto and cross spectra - verification and adjustments based on tu1441 data. Technical report, 2005.

[31] Goody M. Empirical spectral model of surface pressure fluctuations. AIAA J., 42:1788-1794, 2004.

[32] Goody M. and Simpson R.L. An experimental investigation of pressure fluctuations in three-dimensional turbulent boundary layers. Technical report, 1999.

[33] Gravante S.P., Naguib A.M., Wark C.E., and Nagib H.M. Characterization of the pressure fluctuations under a fully developed turbulent boundary layer. AIAA J., 36:1808-1816, 1998.

[34] Bull M. Properties of the fluctuating wall-pressure field of a turbulent boundary layer. Technical report, 1963.

[35] Blake W.K. Turbulent boundary-layer wall-pressure fluctuations on smooth and rough walls. J. Fluid Mech., 44:637-660, 1970.

[36] Hwang Y.F., Bonness W.K., and Hambric S.A. Comparison of semi-empirical models for turbulent boundary layer wall pressure spectra. J. Sound Vib., 319:199-217, 2009.

[37] Schewe G. On the structure and resolution of wall-pressure fluctuations associated with turbulent boundary-layer flow. J. Fluid Mech., 134:311-328, 1983. 
[38] McGrath B.E. and Simpson R.L. Some features of surface pressure fluctuations in turbulent boundary layers with zero and favourable pressure gradients. Technical report, 1987.

[39] Olivero-Bally P., Forestier B.E., Focquenoy E., and Olivero P. Wall-pressure fluctuations in natural and manipulated turbulent boundary layers in air and water. Flow Noise Modeling, Measurement, and Control.

[40] Leclerq D.J.J. and Bohineust X. Investigation and modelling of the wall pressure field beneath a turbulent boundary layer at low and medium frequencies. J. Sound Vib., 257:477-501, 2002.

[41] Miller T.S. Turbulent Boundary Layer Models for Acoustic Analysis. PhD thesis, Wichita State University, 2002.

[42] Salze E., Bailly C., Marsden O., Jondeau E., and Juvé D. Wavelet analysis to detect multi-scale coherent eddy structures and intermittency in turbulent boundary layers. 20th AIAA/CEAS Aeroacoustics Conference, Atlanta, GA, pages $1-26$, 2014.

[43] Van Blitterswyk J. and Rocha J. Prediction and measurement of flow-induced wallpressure fluctuations at low mach numbers. Canadian Acoustics, 42:13-25, 2014.

[44] Kraichnan R.H. Pressure fluctuations in a turbulent flow over a flat plate. J. Acoust. Soc. Am., 28:378-390, 1956.

[45] Phillips O.M. On the aerodynamic surface sound from a plane turbulent boundary layer pressure fluctuations in a turbulent flow over a flat plate. Proc. Roy. Soc. Lond., A234:327-335, 1956. 
[46] Lilley G.M. Wall pressure fluctuations under turbulent boundary layers at subsonic and supersonic speeds. Technical report, 1963.

[47] Smol'yakov A.V. Calculation of the spectra of pseudosound wall-pressure fluctuations in turbulent boundary layers. Acoust. Phys., 46:342-347, 2000.

[48] Panton R.L. and Linebarger J.H. Wall pressure spectra calculations for equilibrium boundary layers. J. Fluid Mech., 65:261 - 287, 1974.

[49] Robin O., Moreau S., Padois T., and Berry A. Measurement of the wavenumberfrequency spectrum of wall pressure fluctuations: spiral-shaped rotative arrays with pinhole-mounted quarter inch microphones. 19th AIAA/CEAS Aeroacoustics Conference, Berlin, Germany, pages 1 - 18, 2013.

[50] Panton R.L., Goldman A.L., Lowery R.L., and Reishman R.R. Low-frequency pressure fluctuations in axisymmetric turbulent boundary layers. J. Fluid Mech., $97: 299-319,1980$.

[51] Blake W.K. Mechanics of Flow-Induced Sound and Vibration. Academic Press Inc., London, 1986.

[52] Efimtsov B. Characteristics of the field of turbulent wall pressure fluctuations at large Reynolds numbers. Sov. Phys. Acoust., 28:289-292, 1982.

[53] Efimtsov B. The prediction of the pressure fluctuation field characteristics of the TBL. Technical report, 1995.

[54] Robertson J. Prediction of in-flight fluctuating pressure environments including proturbence induced flow. Technical report, 1971.

[55] Laganelli A. and Wolfe H. Prediction of fluctuating pressure in attached and separated turbulent boundary-layer flow. J. Aircraft, 30:962-970, 1993. 
[56] Dussauge J.P., Smith R.W., Smits A.J., Fernholz H., Finley P.J., and Spina E.F. Turbulent boundary layers in subsonic and supersonic flow. Technical report, 1996.

[57] Chacin J.M. and Cantwell B.J. Dynamics of low Reynolds number turbulent boundary layer. J. Fluid Mech., 404:87-115, 2000.

[58] Bradshaw P. The turbulence structure of equilibrium boundary layers. J. Fluid Mech., 29:625-645, 1967.

[59] Bradshaw P. 'Inactive' motion and pressure fluctuations in turbulent boundary layers. J. Fluid Mech., 30:241-258, 1967.

[60] Burton T.E. Wall pressure fluctuations at smooth and rough surfaces under turbulent boundary layers with favourable and adverse pressure gradients. Technical report, 1973.

[61] Coles D.E. The turbulent boundary layer in a compressible fluid. Technical report, 1962.

[62] Geib Jr. F.E. Measurements on the effect of transducer size on the resolution of boundary-layer pressure fluctuations. Acoustical Society of America, 46:253-261, 1969.

[63] Lupetow R. M. Transducer resolution and the turbulent wall pressure spectrum. J. Acoust. Soc. Am., 97:370-378, 1995.

[64] Miller T.S., Gallman J.M., and Moeller M.J. Review of turbulent boundary-layer models for acoustic analysis. J. Aircraft, 49:1739-1754, 2012.

[65] Thomas A.S.W. and Bull M.K. On the role of wall-pressure fluctuations in deterministic motions in the turbulent boundary layer. J. Fluids Mech., 128:283-322, 1983. 
[66] Gloerfelt X. The link between wall pressure spectra and radiated sound from turbulent boundary layers. 16th AIAA/CEAS Aeroacoustics Conference, Stockholm, Sweden, pages $1-30,2010$.

[67] Van Blitterswyk J. and Rocha J. Wall-pressure coherence beneath a low Reynolds number turbulent boundary layer. 24th ICTAM, Montreal, Canada, pages 1-2, 2016.

[68] Wilczynski V. and Casarella M.J. Influences of near-wall and induced irrotational motion in a turbulent boundary layer on wall-pressure fluctuations. Trans. ASME, 117:252-262, 1995.

[69] Meinhart C.D. and Adrian R.J. On the existence of uniform momentum zones in a turbulent boundary layer. Phys. Fluids, 7:694-696, 1995.

[70] Johansson A.V., Her J-Y., and Haritonidis J.H. On the generation of high-amplitude wall-pressure peaks in turbulent boundary layers and spots. J. Fluid Mech., 175:119-142, 1987.

[71] Kobayashi Y. and Ichiro M. Wall pressure and its relation to turbulent structure of a boundary layer. Exp. Fluids, 4:49-55, 1986.

[72] Naguib A.M., Wark C.E., and Juckenhöfel. Stochastic estimation and flow sources associated with surface pressure events in a turbulent boundary layer. Phys. Fluids, 13:2611-2626, 2001.

[73] Karangelen C.C., Wilczynski V., and Casarella M.J. Large amplitude wall pressure events beneath a turbulent boundary layer. J. Fluids Eng., 115:653-659, 1986.

[74] Snarski S.R. and Lupetow R.M. Wall pressure and coherent structures in a turbulent boundary layer on a cylinder in axial flow. J. Fluid Mech., 286:137-171, 1995. 
[75] Guezennec Y.G. Documentation of large coherent structures associated with wall events in turbulent boundary layers. PhD thesis, Illinois Institute of Technology, 1985.

[76] Johansson A.V. and Alfredsson P.H. On the structure of turbulent channel flow. J. Fluid Mech., 122:295-314, 1982.

[77] De Ojeda W. and Wark C.E. Instantaneous velocity and wall pressure features in a turbulent boundary layer. Technical report, 1997.

[78] Marusic I. On the role of large-scale structures in wall turbulence. Phys. Fluids, $13: 735-743,2001$.

[79] Ganapathisubramani B., Hutchins N., Hambleton W.T., Longmire E.K., and Marusic I. Investigation of large-scale coherence in turbulent boundary layers using two-point correlations. J. Fluid Mech., 524:57-80, 2005.

[80] Tomkins C.D. and Adrian R.J. Spanwise structures and scale growth in turbulent boundary layers. J. Fluid Mech., 490:37-74, 2003.

[81] Townsend A.A. The Structure of Turbulent Shear Flow. Cambridge University Press, 2003.

[82] Lee J.H. and Sung H.J. Very-large-scale motions in a turbulent boundary layer. J. Fluid Mech., 673:80-120, 2011.

[83] Rathnasingham R. and Breuer K.S. Active control of turbulent boundary layers. J. Fluid Mech., 495:209-233, 2003.

[84] Onorato M., Camussi R., and Iuso G. Small scale intermittency and bursting in a turbulent channel flow. Phys. Rev. E, 61:1447-1454, 2000. 
[85] Roberts S.K. Boundary Layer Transition in Attached and Separated Flows at Low Reynolds Numbers. PhD thesis, Carleton University, 2005.

[86] Bruun H.H. Hot-wire Anemometry: Principles and Signal Analysis. Oxford Science Publications, Oxford, 1987.

[87] Efimtsov B.M., Golubev A.Yu., Kuznetsov V.B., Rizzi S.A., Anderson A.O., Rackl R.G., and Andrianov E.V. Effect of transducer flushness on measured surface pressure fluctuations in flight. 43rd AIAA Aerospace Sciences Meeting and Exhibit, Reno, Nevada, pages 1-16, 2005.

[88] Masson P.J., Leveque J., Netter D., and Rezzoug A. Effect of transducer size, shape, and surface sensitivity on the measurement of boundary-layer pressures. Acoustical Society of America, 41:1358-1363, 1967.

[89] Simpson R., Ghodbane M., and McGrath B. Surface pressure fluctuations in a separating turbulent boundary layer. J. Fluid Mech., 177:167 - 186, 1987.

[90] Forest J.B. The Wall Pressure Spectrum of High Reynolds Number Rough-Wall Turbulent Boundary Layers. PhD thesis, Virginia Polytechnic Institute and State University, 2012.

[91] Naguib A.M., Gravante S.P., and Wark C.E. Extraction of turbulent wall-pressure time-series using an optimal filtering scheme. Exp. Fluids, 22:14-22, 1996.

[92] Braslow A.L. Technical report.

[93] Rona A. and Soueid H. Boundary layer trips for low Reynolds number wind tunnel tests. 48th AIAA Aerospace Sciences Meeting Including the New Horizons Forum and Aerospace Exposition, Orlando, Florida, pages 1-13, 2010. 
[94] Forest J.B. Effects of Reynolds number on the structure of turbulent boundary layers. PhD thesis, Princeton University, 1994.

[95] Finley P.J. and Fernholz H.H. A critical compilation of compressible turbulent boundary layer data. Technical report, 1977.

[96] White F.M. Fluid Mechanics, 3rd Ed. McGraw-Hill Education, United States of America, 2005.

[97] Compton D.A. and Eaton J.K. Near-wall measurements of a three-dimensional turbulent boundary layer. Technical report, 1995.

[98] Jorgensen F.E. How to measure turbulence with hot-wire anemometers - a practical guide. Technical report, 2002.

[99] Elishakoff I. Probabilistic Theory of Structures, 2nd Ed. Dover Publications, 1999.

[100] Snarski S.R. Relation between the fluctuating wall pressure and the turbulent structure of a boundary layer on a cylinder in axial flow. Technical report, 1993.

[101] Oppenheim A. V., Schafer R.W., and Buck J. R. Discrete-Time Signal Processing, 2nd Ed. Prentice Hall, New Jersey, 1999.

[102] Lowson M. Prediction of boundary layer pressure fluctuations. Technical report, 1968.

[103] Bies D. A review of flight and wind tunnel measurements of boundary layer pressure fluctuations and induced structural response. Technical report, 1965.

[104] Chase D.M. Modeling the wavevector-frequency spectrum of turbulent boundary layer wall pressure. J. Sound Vib., 70:29-67, 1980. 
[105] Smol'yakov A.V. A new model for the cross spectrum and wavenumber-frequency spectrum of turbulent pressure fluctuations in a boundary layer. Acoust. Phys.+, 52:331-337, 2006.

[106] Singer B.A. New model for off-axis cross-spectral density. Technical report, 1996.

[107] Clauser F.H. The turbulent boundary layer. Adv. Appl. Mech., 4:1-54, 1956.

[108] Castillo L. and Wang X. Similarity analysis for turbulent boundary layer with pressure gradient. Trans. ASME, J. Fluid Eng., 126:827-834, 2004.

[109] Smits A.J., Matheson N., and Joubert P.N. Low-Reynolds-number turbulent boundary layers in zero and favourable pressure gradients. J. Ship Research, 27:147-157, 1983.

[110] Hutchins H. and Marusic I. Evidence of very long meandering features in the logarithmic region of turbulent boundary layers. J. Fluid Mech., 579:1-28, 2007.

[111] Adrian R. J. Hairpin vortex organization in wall turbulence. Phys. Fluids, 19:041301-1-041301-16, 2007.

[112] Zhou J., Adrian R.J., Balachandar S., and Kendall T.M. Mechanisms for generating coherent packets of hairpin vortices in channel flow. J. Fluid Mech., 387:353-396, 1999.

[113] Bradshaw P. Irrotational fluctuations near a turbulent boundary layer. J. Fluid Mech., 27:209-230, 1967.

[114] Kim J., Choi J.-I., and Sung H. J. Relationship between wall pressure fluctuations and streamwise vortices in a turbulent boundary layer. Phys. Fluids, 14:898-901, 2002. 
[115] Tang Z-Q., Jiang N., Schröder, and Geisler R. Tomographic piv investigation of coherent structures in a turbulent boundary layer flow. Acta Mech. Sin., 28:pp. 572-582, 2012. 
Appendix A

\section{Properties of Detected Event Packets}




\begin{tabular}{|c|c|c|c|c|c|c|c|c|c|}
\hline \multirow[t]{2}{*}{ Event Packet } & \multirow[t]{2}{*}{ Microphone } & \multirow{2}{*}{$x^{+}=\frac{\Delta t U_{c} v}{U_{\tau}}$} & \multirow{2}{*}{$U_{c}$} & \multicolumn{6}{|c|}{ Number of Events } \\
\hline & & & & Type 1 & Type 2 & Type 3 & Type 4 & Type 5 & Type 6 \\
\hline \multicolumn{10}{|c|}{ Configuration A (Fig. $4.14 \mathrm{a}$} \\
\hline \multirow{4}{*}{ Al } & 1 & 1,342 & 0.82 & 1 & 2 & 4 & 4 & 3 & 2 \\
\hline & 2 & 1,306 & 0.73 & 1 & 2 & 4 & 8 & 6 & 6 \\
\hline & 3 & 696 & 0.67 & 1 & 4 & 4 & 5 & 5 & 3 \\
\hline & 4 & 754 & 0.52 & 2 & 5 & 5 & 8 & 7 & 0 \\
\hline \multirow{3}{*}{ A2 } & 1 & 934 & 0.70 & 1 & 1 & 2 & 2 & 1 & 1 \\
\hline & 2 & 1,026 & 0.63 & 1 & 3 & 3 & 2 & 3 & 2 \\
\hline & 3 & 1,027 & 0.63 & 0 & 2 & 3 & 2 & 3 & 3 \\
\hline \multirow{3}{*}{ A3 } & 1 & 1,219 & 0.61 & 1 & 1 & 2 & 3 & 2 & 2 \\
\hline & 2 & 1,102 & 0.61 & 1 & 2 & 6 & 6 & 5 & 4 \\
\hline & 3 & 1,678 & 0.74 & 1 & 4 & 7 & 6 & 8 & 4 \\
\hline \multirow{3}{*}{$\mathrm{A} 4$} & 2 & 485 & 0.68 & 1 & 1 & 0 & 0 & 0 & 0 \\
\hline & 3 & 748 & 0.65 & 2 & 1 & 3 & 5 & 2 & 1 \\
\hline & 4 & 917 & 0.69 & 2 & 4 & 3 & 3 & 2 & 0 \\
\hline \multirow{3}{*}{ A5 } & 2 & 544 & 0.81 & 0 & 2 & 2 & 0 & 0 & 0 \\
\hline & 3 & 569 & 0.73 & 0 & 1 & 2 & 1 & 1 & 0 \\
\hline & 4 & 917 & 0.64 & 1 & 3 & 4 & 4 & 2 & 0 \\
\hline A6 & 1 & 625 & 0.71 & 2 & 1 & 2 & 1 & 1 & 1 \\
\hline A7 & 1 & 610 & 0.81 & 1 & 0 & 1 & 2 & 2 & 0 \\
\hline A8 & 2 & 195 & 0.66 & 1 & 1 & 1 & 2 & 1 & 0 \\
\hline A9 & 3 & 438 & 0.49 & 1 & 1 & 2 & 2 & 2 & 2 \\
\hline A9 & 3 & 690 & 0.72 & 1 & 3 & 2 & 4 & 2 & 1 \\
\hline Al0 & 3 & 559 & 0.80 & 0 & 1 & 2 & 1 & 1 & 0 \\
\hline Al1 & 3 & 416 & 0.70 & 1 & 2 & 2 & 2 & 0 & 0 \\
\hline \multirow[t]{2}{*}{$\mathrm{A} 12$} & 4 & 592 & 0.80 & 0 & 2 & 2 & 1 & 0 & 0 \\
\hline & \multicolumn{4}{|c|}{ Configuration B (Fig. } & $4.14 \mathrm{~b}$ & & & & \\
\hline \multirow{3}{*}{$\mathrm{B} 1$} & 1 & 922 & 0.83 & 1 & 1 & 2 & 1 & 1 & 1 \\
\hline & 2 & 630 & 0.80 & 0 & 1 & 3 & 2 & 3 & 3 \\
\hline & 3 & 2,886 & 0.82 & 1 & 4 & 6 & 6 & 8 & 6 \\
\hline \multirow{2}{*}{ B2 } & 1 & 1,784 & 0.78 & 2 & 2 & 3 & 5 & 4 & 2 \\
\hline & 2 & 1,017 & 0.77 & 1 & 1 & 3 & 2 & 3 & 3 \\
\hline \multirow{2}{*}{ B3 } & 3 & 2,192 & 0.77 & 2 & 6 & 7 & 3 & 5 & 5 \\
\hline & 4 & 2,640 & 0.79 & 3 & 6 & 8 & 5 & 7 & 8 \\
\hline B4 & 1 & 1,303 & 0.55 & 0 & 1 & 5 & 5 & 3 & 2 \\
\hline B5 & 2 & 518 & 0.63 & 0 & 0 & 2 & 3 & 3 & 3 \\
\hline
\end{tabular}

Table A.1: Summary of event packets detected using higher-order statistics for $R e_{\theta}=$ 1,886 


\begin{tabular}{|c|c|c|c|c|c|c|c|c|c|}
\hline \multirow[t]{2}{*}{ Event Packet } & \multirow[t]{2}{*}{ Microphone } & \multirow{2}{*}{$x^{+}=\frac{\Delta t U_{c} v}{U_{\tau}}$} & \multirow[t]{2}{*}{$U_{c}$} & \multicolumn{6}{|c|}{ Number of Events } \\
\hline & & & & Type 1 & Type 2 & Type 3 & Type 4 & Type 5 & Type 6 \\
\hline & & & figura & on A (Fig. & $4.14 \mathrm{a}$ & & & & \\
\hline \multirow{4}{*}{$\mathrm{Al}$} & 1 & 1,478 & 0.53 & 1 & 2 & 2 & 3 & 2 & 1 \\
\hline & 2 & 1,855 & 0.72 & 1 & 3 & 3 & 5 & 5 & 3 \\
\hline & 3 & 1,801 & 0.72 & 1 & 4 & 3 & 5 & 5 & 1 \\
\hline & 4 & 1,625 & 0.81 & 1 & 2 & 3 & 4 & 5 & 1 \\
\hline \multirow{4}{*}{ A2 } & 1 & 2,150 & 0.61 & 1 & 3 & 5 & 5 & 4 & 4 \\
\hline & 2 & 2,910 & 0.81 & 0 & 5 & 4 & 9 & 8 & 6 \\
\hline & 3 & 2,660 & 0.66 & 0 & 4 & 6 & 7 & 6 & 7 \\
\hline & 4 & 2,075 & 0.63 & 1 & 4 & 5 & 10 & 9 & 2 \\
\hline \multirow{4}{*}{ A3 } & 1 & 1,482 & 0.70 & 1 & 2 & 4 & 5 & 4 & 8 \\
\hline & 2 & 1,742 & 0.79 & 1 & 2 & 3 & 5 & 5 & 5 \\
\hline & 3 & 1,344 & 0.67 & 1 & 3 & 3 & 4 & 3 & 3 \\
\hline & 4 & 1,274 & 0.93 & 1 & 1 & 2 & 2 & 2 & 0 \\
\hline \multirow{4}{*}{ A4 } & 1 & 1,769 & 0.66 & 1 & 3 & 6 & 4 & 3 & 2 \\
\hline & 2 & 1,895 & 0.69 & 1 & 4 & 4 & 8 & 9 & 4 \\
\hline & 3 & 1,344 & 0.67 & 1 & 3 & 3 & 4 & 3 & 3 \\
\hline & 4 & 2,497 & 0.69 & 1 & 7 & 7 & 9 & 11 & 6 \\
\hline \multirow{3}{*}{ A5 } & 1 & 1,819 & 0.84 & 1 & 2 & 5 & 3 & 2 & 3 \\
\hline & 2 & 1,537 & 0.75 & 1 & 3 & 5 & 4 & 2 & 2 \\
\hline & 3 & 736 & 0.58 & 1 & 2 & 2 & 2 & 2 & 2 \\
\hline \multirow{2}{*}{ A6 } & 1 & 2,272 & 0.65 & 2 & 3 & 6 & 6 & 7 & 7 \\
\hline & 2 & 2,472 & 0.66 & 0 & 4 & 7 & 8 & 12 & 9 \\
\hline A7 & 3 & 2,250 & 0.69 & 1 & 4 & 5 & 6 & 7 & 6 \\
\hline A8 & 4 & 2,643 & 0.71 & 0 & 6 & 6 & 3 & 4 & 1 \\
\hline A9 & 4 & 2,301 & 0.73 & 0 & 8 & 4 & 10 & 10 & 0 \\
\hline \multicolumn{10}{|c|}{ Configuration B (Fig. $4.14 \mathrm{~b}$} \\
\hline \multirow{4}{*}{ B1 } & 1 & 605 & 0.58 & 1 & 2 & 2 & 1 & 0 & 0 \\
\hline & 2 & 1,499 & 0.62 & 1 & 3 & 3 & 3 & 3 & 2 \\
\hline & 3 & 2,468 & 0.79 & 2 & 4 & 6 & 5 & 9 & 8 \\
\hline & 4 & 2,030 & 0.70 & 1 & 2 & 4 & 7 & 6 & 6 \\
\hline \multirow{4}{*}{ B2 } & 1 & 2,878 & 0.71 & 2 & 4 & 2 & 5 & 5 & 5 \\
\hline & 2 & 2,275 & 0.77 & 0 & 2 & 6 & 6 & 7 & 6 \\
\hline & 3 & 4,133 & 0.80 & 2 & 5 & 8 & 9 & 12 & 12 \\
\hline & 4 & 2,411 & 0.79 & 0 & 2 & 5 & 4 & 4 & 3 \\
\hline \multirow{3}{*}{ B3 } & 1 & 3,440 & 0.81 & 1 & 4 & 5 & 3 & 5 & 4 \\
\hline & 2 & 4,206 & 0.83 & 3 & 6 & 8 & 5 & 7 & 8 \\
\hline & 3 & 2,445 & 0.68 & 1 & 4 & 2 & 5 & 6 & 0 \\
\hline B4 & 4 & 1,284 & 0.87 & 1 & 1 & 4 & 6 & 4 & 5 \\
\hline
\end{tabular}

Table A.2: Summary of select event packets detected using higher-order statistics for $\operatorname{Re}_{\theta}=2,546$ 